\title{
The INTEGRAL Galactic bulge monitoring program: the first 1.5 years ${ }^{\star}$
}

\author{
E. Kuulkers ${ }^{1}$, S. E. Shaw ${ }^{2,3}$, A. Paizis ${ }^{4}$, J. Chenevez ${ }^{5}$, S. Brandt ${ }^{5}$, T. J.-L. Courvoisier ${ }^{3,6}$, A. Domingo ${ }^{7}$, K. Ebisawa ${ }^{8}$, \\ P. Kretschmar ${ }^{1}$, C. B. Markwardt ${ }^{9,10}$, N. Mowlavi ${ }^{3}$, T. Oosterbroek ${ }^{11}$, A. Orr ${ }^{12}$, \\ D. Rísquez ${ }^{7}$, C. Sanchez-Fernandez ${ }^{1}$, and R. Wijnands ${ }^{13}$
}

\author{
1 ISOC, ESA/ESAC, Urb. Villafranca del Castillo, PO Box 50727, 28080 Madrid, Spain \\ e-mail: Erik.Kuulkers@esa.int \\ 2 School of Physics and Astronomy, University of Southampton, SO17 1BJ Southampton, UK \\ 3 INTEGRAL Science Data Centre (ISDC), 16 Chemin d'Ecogia, 1290 Versoix, Switzerland \\ 4 INAF-IASF, Sezione di Milano, via Bassini 15, 20133 Milano, Italy \\ 5 Danish National Space Center, Juliane Maries Vej 30, 2100 Copenhagen, Denmark \\ ${ }^{6}$ Observatoire de Genève, 51 Chemin des Mailletes, 1290 Sauverny, Switzerland \\ 7 Laboratorio de Astrofísica Espacial y Física Fundamental, INTA, Apartado 50727, 28080 Madrid, Spain \\ 8 Center for PLAnning and INformation systems, Institute of Space and Astronautical Science, Yoshinodai 3-1-1 Sagamihara, \\ Kanagawa, 229-8510, Japan \\ 9 Department of Astronomy, University of Maryland, College Park, MD 20742, USA \\ 10 X-ray Astrophysics Laboratory, Mail Code 662, NASA Goddard Space Flight Center, Greenbelt, MD 20771, USA \\ 11 Science Payload and Advanced Concepts Office, ESA-ESTEC, Postbus 299, 2200 AG, Noordwijk, The Netherlands \\ 12 Research and Scientific Support Department, ESA-ESTEC, Postbus 299, 2200 AG, Noordwijk, The Netherlands \\ 13 Astronomical Institute "Anton Pannekoek", University of Amsterdam, Kruislaan 403, 1098 SJ Amsterdam, The Netherlands
}

Received 27 October 2006 / Accepted 28 December 2006

\section{ABSTRACT}

Aims. The Galactic bulge region is a rich host of variable high-energy point sources. Since 2005, February 17 we are monitoring the source activity in the Galactic bulge region regularly and frequently, i.e., about every three days, with the instruments onboard INTEGRAL. Thanks to the large field of view, the imaging capabilities and the sensitivity at hard X-rays, we are able to present for the first time a detailed homogeneous (hard) X-ray view of a sample of 76 sources in the Galactic bulge region.

Methods. We describe the successful monitoring program and show the first results from the start of the monitoring up to 2006, April 21, i.e., for a period of about one and a half year, during three visibility seasons. We focus on the short (hour), medium (month) and long-term (year) variability in the hard X-ray bands, i.e., $20-60 \mathrm{keV}$ and $60-150 \mathrm{keV}$. When available, we discuss the simultaneous observations in the soft X-ray, $3-10 \mathrm{keV}$ and $10-25 \mathrm{keV}$, bands.

Results. Almost all the sources in the Galactic bulge region we detect in the $20-60 \mathrm{keV}$ and $60-150 \mathrm{keV}$ bands are variable. During the last two and a half weeks of the third visibility season most of the known persistent (hard) X-ray sources in the Galactic Center region were not detected. Of our sample of sources, per visibility season we detect 32/33 sources in the 20-60 keV band and 8/9 sources in the $60-150 \mathrm{keV}$ band above a signal to noise of 7 . On average, we find per visibility season one active bright $\gtrsim 100 \mathrm{mCrab}$, 20-60 keV) black-hole candidate X-ray transient and three active weaker ( $\$ 25 \mathrm{mCrab}, 20-60 \mathrm{keV})$ neutron star X-ray transients. Most of the time a clear anti-correlation can be seen between the soft and hard X-ray emission in some of the X-ray bursters. Hard $\mathrm{X}$-ray flares or outbursts in X-ray bursters, which have a duration of the order of weeks are accompanied by soft X-ray drops. On the other hand, hard X-ray drops can be accompanied by soft X-ray flares/outbursts. During the course of our program we found a number of new sources, IGR J17354-3255, IGR 17453-2853, IGR J17454-2703, IGR J17456-2901b, IGR J17536-2339, and IGR J17541-2252. We report here on some of the high-energy properties of these sources.

Conclusions. The high-energy light curves of all the sources in the field of view, and the high-energy images of the region, are made available through the WWW, as soon as possible after the observations have been performed, at http://isdc. unige.ch/Science/BULGE/.

Key words. accretion, accretion disks - binaries: close - binaries: general - stars: neutron - Galaxy: bulge - X-rays: binaries

\section{Introduction}

The bulge of our Galaxy hosts a variety of hard X-ray and $\gamma$-ray point sources (e.g., Knight et al. 1985; Skinner et al. 1987; Churazov et al. 1994; see, e.g., Bird et al. 2006; Bélanger et al. 2006; Revnivtsev et al. 2004a, for observations made by INTEGRAL, the International Gamma-Ray Astrophysics

* Appendices are only available in electronic form at http://www . aanda.org
Laboratory; Winkler et al. 2003). Among them are persistent and transient neutron-star and black-hole (candidate) binaries, as well as magnetic cataclysmic variables and AGN. Due to the variability these sources possess on time scales of milliseconds to days (quasi-periodic oscillations, pulsations, (absorption) dips, eclipses, type I and type II X-ray bursts, orbital variations, flares) and weeks to years (orbital variations, outburst cycles, on/off states), the region never looks exactly the same. 
Hard X-ray ( $\gtrsim 20 \mathrm{keV})$ emission emerging from the Galactic bulge sources mainly comes from highly energetic processes occurring in the course of accretion from a donor star on to the compact object, i.e., a white dwarf, neutron star or black hole. Since most of the emission comes from the region close to these compact objects, studying the hard X-ray and $\gamma$-ray emission will give us more insight into the accretion processes under extreme conditions, as well as possibly identifying the nature of the compact accretor.

From 17 February 2005 onwards, whenever the Galactic bulge region was visible by INTEGRAL, we have been monitoring this region approximately every 3 days. This paper serves mainly as a description of this program in more detail and we show the first results, spanning times scales between half an hour and one and a half year. Preliminary announcements of some of these results were made by Bodghee et al. (2005), Brandt et al. (2005), Chenevez et al. (2006a,b), Kretschmar et al. (2005), Kuulkers et al. (2005a,b, 2006a), Mowlavi et al. (2005), Shaw et al. (2005a,b,c, 2006) and Turler et al. (2006). A preliminary report of our program was presented by Kuulkers et al. (2006b).

\section{INTEGRAL and the Galactic bulge monitoring program}

Since X-rays typically above $10 \mathrm{keV}$ are difficult to focus using currently known reflecting material, one has to revert to other means of imaging techniques when investigating crowded regions. Coded masks are effective imagers in this energy range, as, e.g., shown by the first hard X-ray $(20-30 \mathrm{keV})$ images of the region around the Galactic Center (Skinner et al. 1987).

INTEGRAL is an ESA scientific mission dedicated to fine spectroscopy $(E / \Delta E \simeq 500$; SPI; Vedrenne et al. 2003) and fine imaging (angular resolution: 12' FWHM, source location accuracy: $\simeq 1-3^{\prime}$; IBIS; Ubertini et al. 2003) of celestial $\gamma$-ray sources in the energy range $15 \mathrm{keV}$ to $10 \mathrm{MeV}$ with simultaneous monitoring in the X-ray (3-35 keV, angular resolution: $3^{\prime}$; JEM-X; Lund et al. 2003) and optical ( $V$-band, $550 \mathrm{~nm}$; OMC; Mas-Hesse et al. 2003) energy ranges. All the instruments onboard INTEGRAL, except the OMC, have coded masks.

INTEGRAL has already spent a considerable amount of observing time on the Galactic bulge region, providing a deep insight (see, e.g., Bélanger et al. 2006). Our program, however, was initiated to monitor, for the first time with INTEGRAL, this region frequently on a regular basis at hard X-rays and $\gamma$-rays. The main aim is to investigate the source variability and transient activity on time scales of hours to days to weeks to months simultaneously at relatively soft $(\lesssim 10 \mathrm{keV})$ and hard $(\gtrsim 10 \mathrm{keV})$ energies. One complete hexagonal dither pattern (7 pointings of $\simeq 1800$ s each, i.e., 1 on-axis pointing, 6 off-source pointings in a hexagonal pattern around the nominal target location, each $2^{\circ}$ apart) is performed during each INTEGRAL revolution (orbit) around the earth, i.e., roughly every 3 days. This yields a total coverage around the Galactic Center of $29^{\circ}$ with IBIS/ISGRI and about $6^{\circ}$ with JEM-X for a total exposure of $12.6 \mathrm{ks}$. With the fully and partially coded field of view we cover about half of the Galactic bulge X-ray binary population with IBIS/ISGRI (see also, e.g., in 't Zand 2001; in 't Zand et al. 2004).

The hexagonal dither pattern is done whenever the region is visible by INTEGRAL (about two times per year for a total period of about 4 months). We refer to each visibility period as "season"; in this paper we report on observations made during three seasons. As a service to the scientific community, the JEM-X light curves (3-10 keV and $10-25 \mathrm{keV}$ ) and the IBIS/ISGRI light curves (20-60 keV and 60-150 keV) are made publicly available as soon as possible after the observations are performed, both in graphic and in ASCII form. In addition, IBIS/ISGRI and JEM-X mosaic images (i.e., composite images made of overlapping images) of each hexagonal dither observation are provided, with information on the detected sources. Finally, all IBIS/ISGRI $20-60 \mathrm{keV}$ mosaic images per revolution are stacked into a movie, showing the ever-changing hard X-ray $/ \gamma$-ray sky. The results, as well as more information about the program, can be retrieved from the INTEGRAL Galactic bulge Monitoring WWW page hosted at the INTEGRAL Science Data Center (ISDC) in Switzerland: http://isdc.unige.ch/Science/BULGE/.

Similar Galactic bulge monitoring programs at various soft and hard X-ray energies have been performed (see, e.g., in 't Zand 2001). For example, GRANAT/SIGMA (30 keV-1 MeV) performed regular observations of the Galactic Center region between 1990 and 1994 in comparable energy ranges, i.e., above $>35 \mathrm{keV}$, and with comparable visibility periods throughout the year (see, e.g., Churazov et al. 1993). The sensitivity, angular resolution and source location accuracy of GRANAT/SIGMA (respectively, $5 \sigma$ detection level of $\simeq 10 \mathrm{mCrab}$ at $40-100 \mathrm{keV}$ for an exposure time of $\simeq 9 \mathrm{Ms}$ at the Galactic Center, $\simeq 15^{\prime}$ and $\simeq 2-3^{\prime}$; see Revnivtsev et al. $2004 \mathrm{~b}$ ) are, however, not as good as INTEGRAL/IBIS ( $5 \sigma$ detection level of $\simeq 1 \mathrm{mCrab}$ at $40-100 \mathrm{keV}$ for an exposure time of $\simeq 1.5 \mathrm{Ms}$ at the Galactic Center, see Bird et al. 2006).

Some monitoring programs are currently ongoing, such as the RXTE Galactic bulge Scans (Swank \& Markwardt 2001; Markwardt 2006). However, the RXTE/PCA $(2-60 \mathrm{keV})$ and HEXTE $(15-250 \mathrm{keV})$ do only have a $2^{\circ}$ collimator and no imaging resolution, providing only information on a given source for a short time when the instrument scans over it; moreover, in the Galactic Center region itself there is significant source confusion. There are currently other instruments in operation at similar energy ranges as those covered by IBIS/ISGRI, such as Swift/BAT (15-150 keV with a field-of view of 2 steradians; Barthelmy 2000). However, they have a worse imaging capability, again leading to source confusion in the Galactic Center region (Swift/BAT PSF angular resolution is $22^{\prime}$ ), and a lower sensitivity ( $3 \sigma$ detection level of $\simeq 27 \mathrm{mCrab}$ at $15-50 \mathrm{keV}$ for an exposure of 736 s; see Krimm et al. 2006).

In this paper we show the results of three full seasons of our AO-3 monitoring, about 1.5 years, i.e., from 005, February 18-April 19 (INTEGRAL revolutions 287-307; MJD 53419-53479), 2005, August 16-October 26 (revolutions 347-370; MJD 53598-54034), and 2006, February 9-April 21 (revolutions 406-429; MJD 53775-53846). We note that the program continues in INTEGRAL AO-4 (see Kuulkers et al. 2006c), and we intend to extend this program into further Announcement of Opportunities with INTEGRAL. In the next Section we explain how the data analysis is done (Sect. 3). We then summarize the observations per season by focusing on the averaged (or mosaiced) images and corresponding source fluxes and detection significances (Sect. 4.1). The long-term hard Xray $(>20 \mathrm{keV})$ light curves of the monitoring program are shown in Sect. 4.2. In Sect. 4.3 we focus on an interesting period when there was almost no activity in the region around the Galactic center. New INTEGRAL sources found by our program are discussed in Sect. 4.4. Results from the monitoring with the OMC are given in Sect. 4.5. Finally, we summarize and make our concluding remarks in Sect. 5. 


\section{Data analysis}

In the current paper we only consider data from IBIS/ISGRI (Lebrun et al. 2003), JEM-X and OMC. We do not consider the data from IBIS/PICsIT (Labanti et al. 2003) or SPI instruments: either the angular resolution is limited (SPI: $2.5^{\circ}$ ) and therefore the various sources in the Galactic bulge region close to each other complicate the analysis (see, e.g., Bouchet et al. 2005), or the sources are too weak to be detected (IBIS/PICsIT, which operates at $\gtrsim 175 \mathrm{keV}$ ).

The INTEGRAL IBIS and JEM-X data reduction is performed using the Off-line Scientific Analysis (OSA; see Courvoisier et al. 2003), v5.1. In our analysis we use an input source catalog, containing a total of 76 sources (see Table 1). Most of these sources had been previously detected by IBIS/ISGRI (see, e.g., Revnivtsev et al. 2004a; Bird et al. 2006). To these sources we added (transient) sources that have been detected in the mean time, as well as those found in the preINTEGRAL era but currently not detected (yet) by INTEGRAL. To make sure that our results do not depend on the selected input catalogue, we compared our results with the ones obtained using the full ISDC Reference Catalog (version 23.0) which contains all known high-energy sources (see Ebisawa et al. 2003); the imaging and light curve results were consistent. The classifications of the sources are mainly based on the information given by Bird et al. (2006), with updates where noted (Table 1). The black-hole (candidate) binaries were selected from McClintock \& Remillard (2006) and Remillard et al. (2006a), except for the new transient source XTE J1817-330 (see Remillard et al. 2006b). The X-ray burster list is based on the list given by in 't Zand et al. (2004), except for XTE J1739-285 (Brandt et al. 2005) and IGR J17254-3257 (Brandt et al. 2006). SLX 1744-299 and SLX 1744-300 are separated by only $\simeq 3^{\prime}$ (Skinner et al. 1990) and therefore inseparable with current hard X-ray instruments like IBIS/ISGRI. $3^{\prime}$ is at the resolution limit of JEM-X and the soft X-ray emission from the two sources is seen to be different from a point source by JEM-X. However, it is not able to separate the emission confidently, when both sources are active. We therefore regard them as one source, SLX 1744-299/300. IGR J17460-3047 was a source reported by Bird et al. (2004), that turned out to be an artefact (Bird et al. 2006). For the time being this source has been included in our list. We can confirm that in our monitoring program it was never detected by either IBIS/ISGRI or JEM-X (see Sect. 4.1).

The data from IBIS/ISGRI are processed until the production of images in the 20-60 and 60-150 keV energy ranges per single pointing ${ }^{1}$. We force the flux extraction of each of the catalogue sources, regardless of the detection significance of the source. This method is essential in order to clean the images from the ghosts of all the active sources in the field, but does not make any threshold selection and all the positive fluxes are recorded (see Goldwurm et al. 2003, for a detailed description of the IBIS analysis software). In order to detect fainter sources, we then mosaic the images from the seven single pointings (i.e., one hexagonal dither observation) and search for all catalog sources, as well as possible new ones. In the case a new source is found, it can be added to the input catalogue, and a re-analysis is necessary to extract its source fluxes. To achieve even higher sensitivity, we also produced one mosaic image per season as well as a global (3 seasons) mosaic image from the whole AO-3 monitoring program.

${ }^{1}$ A single pointing with INTEGRAL is often refered to as a Science Window, or ScW for short.
For JEM-X the analysis is run through the imaging step to the light-curve step. Light curves with a time bin of the same length as each single pointing are produced for every catalog source inside a radius of $5^{\circ}$. The analysis software used to extract light curves has a known problem in crowded fields like the Galactic bulge, as the contribution from bright sources (such as GX 5-1) is not modeled in sufficient detail which affects the results for weak sources (typically less than about $100 \mathrm{mCrab}$ ) close to very bright ones. Although no detailed quantitative analysis has been done yet, comparisons with the results from JEM-X imaging as well as with results from other X-ray instruments in the same energy range, leads us to the conclude that there is an uncertainty in the fluxes by up to a factor of $\simeq 2$. Again, the images from the seven single pointings are mosaiced in order to create the final image. No further automatic source detection is done for the moment at the mosaic level; however, the images are visually examined for possible new sources.

Type I X-ray bursts (see, e.g., Strohmayer \& Bildsten 2006) are mainly seen in the soft X-ray band, since the observed blackbody temperatures are $\simeq 1-3 \mathrm{keV}$. We use JEM-X to search for such events. Per single pointing we compute the average detector count rate and the corresponding standard deviation. Whenever the difference between the count rate in a 1-s bin and the average count rate exceeds four times the standard deviation value, we flag it as a potential start of a burst. Recontructed images and source light curves are generated within the good-time interval covering the burst event, and are visually checked in order to identify the originating source of the event. The light curves are also visually checked to see whether they adhere to the basic characteristics of a type I X-ray burst, i.e., emitting mainly at the lowest energies (e.g., to exclude solar flares), with a light-curve shape consistent with a fast rise - with respect to the decay and exponential-like decay. Whenever the statistics do allow we also check whether the events show evidence for a spectral softening, due to cooling of the neutron surface, during the decay. In the present paper we only briefly mention the occurrence of type I X-ray bursts, for those sources which were in the field of view of JEM-X, when discussing the long-term light curves. A more detailed (time-resolved) burst analysis is in progress (Sanchez-Fernandez et al., in preparation). We here note that an account of type I X-ray bursts, including those seen from sources in the Galactic bulge region, in earlier INTEGRAL data based on IBIS/ISGRI was reported by Chelovekov et al. (2006).

All fluxes used in this paper are given with respect to the Crab count rate in the respective energy ranges (see Appendix A), i.e., in units of mCrab. Our sensitivity for one hexagonal dither observation (i.e., all seven single pointings per revolution combined) is typically between 5 and $15 \mathrm{mCrab}(5 \sigma)$ for both JEM-X and IBIS/ISGRI. The actual sensitivity depends on factors such as source position (fully or partially coded field of view), background (instrument systematics, solar activity), number of pointings actually performed and usable, and energy (instrument response). We refer to Appendix B for more details. Errors in this paper are quoted at the $1 \sigma$ level. When a source is not detected, we provide $3 \sigma$ upper limits, whenever appropriate. The latter are calculated by determining the variance value in the mosaic maps at a given source position, taking the square root of this value and multiply it by 3 . Note that the variances respresent statistical values, and do not include any systematics.

From the end of August up to mid September 2005 (revolutions 349-357; MJD 53605-53630) there was a high solar activity and various solar flares hampered the observations. During some of the observations the instruments were even off (revolutions 349, 355 and 356; MJD 53605, 53624, 53626, 
Table 1. List of sources in the INTEGRAL Galactic bulge monitoring program, ordered by Galactic longitude (from left to right in the HammerAitoff projection, i.e., from $\left.180^{\circ}-0^{\circ}, 360^{\circ}-180^{\circ}\right)$.

\begin{tabular}{|c|c|c|c|c|c|c|c|c|}
\hline Source & Type & Ref. & Fig. & $1_{\text {II }}$ & $\mathrm{b}_{\mathrm{II}}$ & RA & Dec & Comment \\
\hline GX $17+2$ & LMXB, B, Z & 1 & 7 & 16.432 & -0.710 & 181601.4 & -140211 & \\
\hline SAX J1818.6-1703 & $?, \mathrm{~T}$ & 1 & & 14.078 & -0.710 & 181839 & -1703.1 & \\
\hline GX $13+1$ & LMXB, B, A & 1 & 7 & 13.517 & +0.106 & 181431.55 & -170926.7 & \\
\hline PKS 1830-211 & AGN, QSO & 1 & & 12.166 & -5.712 & 183339.89 & -210339.8 & \\
\hline SGR 1806-20 & SGR & 1 & & 9.996 & -0.242 & 180839.32 & -202439.5 & \\
\hline SAX J1805.5-2031 & $?, \mathrm{~T}$ & 2 & & 9.554 & +0.340 & 180534 & -2030.8 & \\
\hline IGR J18027-2016 & HMXB, T, XP & 1 & & 9.417 & +1.044 & 180239.9 & -201713 & SAX J1802.7-2017 \\
\hline GS 1826-24 & LMXB, B & 1 & 7,11 & 9.272 & -6.088 & 182928.2 & -234749 & Ginga $1826-24$ \\
\hline GX 9+1 & LMXB, A & 1 & 10 & 9.077 & +1.154 & 180132.3 & -203144 & \\
\hline GX 9+9 & LMXB, A & 1 & & 8.513 & +9.038 & 173144.2 & -165742 & 3A $1728-169$ \\
\hline 1RXS J175113.3-201214 & $?$ & 1 & & 8.145 & +3.408 & 175113.4 & -201214 & \\
\hline $\mathrm{H} 1745-203$ & LMXB, G, B & 1,3 & & 7.729 & +3.798 & 174853.40 & -202143.0 & in NGC 6440 \\
\hline IGR J17597-2201 & $\mathrm{LMXB}, \mathrm{B}, \mathrm{D}$ & 1 & & 7.581 & +0.775 & 175946 & -2200.9 & XTE J1759-220 \\
\hline XTE J1818-245 & LMXB?, T & 4 & 10 & 7.441 & -4.196 & 181825.2 & -243231 & \\
\hline 1RXS J174607.8-213333 & $?$ & 5 & & 6.367 & +3.734 & 174607.80 & -213333.0 & \\
\hline GX 5-1 & LMXB, Z & 1 & $10,12,13$ & 5.077 & -1.019 & 180108.2 & -250445 & \\
\hline V1223 Sgr & $\mathrm{CV}$ & 1 & & 4.958 & -14.355 & 185502.24 & -310948.5 & $1 \mathrm{H} 1853-312$ \\
\hline GRS 1758-258 & $\mathrm{LMXB}, \mathrm{BHC}$ & 1 & 6 & 4.508 & -1.361 & 180112.3 & -254436 & \\
\hline IGR J17544-2619 & HMXB?, T & 1 & 10,17 & 3.235 & -0.338 & 175425.7 & -261958 & \\
\hline H1820-303 & $\mathrm{LMXB}, \mathrm{G}, \mathrm{B}, \mathrm{A}$ & 1 & 7 & 2.788 & -7.913 & 182340.45 & -302140.1 & 4U 1820-303; in NGC 6624 \\
\hline IGR J17331-2406 & $?, \mathrm{~T}$ & 6 & & 2.607 & +4.927 & 173308 & -2406.8 & \\
\hline $\mathrm{GX} 3+1$ & LMXB, B, A & 1 & 7 & 2.294 & +0.794 & 174756.0 & -263349 & \\
\hline $\mathrm{GX} 1+4$ & Symb, XP & 1,7 & 10,11 & 1.937 & +4.795 & 173202.16 & -244444.0 & \\
\hline XTE J1807-294 & LMXB, T, XP & 1 & & 1.935 & -4.273 & 180659.8 & -292430 & \\
\hline AX J1749.2-2725 & HMXB, XP & 5 & & 1.699 & +0.108 & 174911.6 & -272536 & \\
\hline AX J1749.1-2733 & HMXB? & 8 & & 1.585 & +0.051 & 174909.0 & -273314 & \\
\hline XB 1832-330 & LMXB, G, B, T & 1 & 7 & 1.531 & -11.372 & 183544.1 & -325929 & in NGC 6652 \\
\hline SLX 1735-269 & LMXB, B & 1 & 7 & 0.785 & +2.398 & 173816.00 & -270016.0 & \\
\hline XTE J1748-288 & $\mathrm{LMXB}, \mathrm{T}, \mathrm{BHC}$ & 9 & & 0.676 & -0.222 & 174805.06 & -282825.8 & \\
\hline IGR J17475-2822 & Mol cloud? & 1 & & 0.601 & -0.040 & 174712 & -2826.6 & \\
\hline EXMS B1709-232 & $?$ & 10 & & 0.594 & +9.269 & 171231 & -2321.2 & \\
\hline IGR J17507-2856 & $?, \mathrm{~T}$ & 11 & & 0.576 & -0.959 & 175044 & -2856.3 & \\
\hline Oph Cluster & Cluster & 1 & & 0.564 & +9.272 & 171226.0 & -232233 & \\
\hline IGR J17419-2802 & $?, \mathrm{~T}$ & 12 & & 0.345 & +1.164 & 174156.0 & -280154 & \\
\hline 1E 1743.1-2843 & LMXB & 1 & 10 & 0.251 & -0.026 & 174619.20 & -284407.0 & \\
\hline SAX J1747.0-2853 & LMXB, B, T & 1 & 7 & 0.207 & -0.239 & 174702.60 & -285258.9 & \\
\hline IGR J17407-2808 & $?, \mathrm{~T}$ & 13 & & 0.115 & +1.341 & 1740.7 & -2808 & \\
\hline SLX 1737-282 & LMXB, B & 1 & 7 & 359.995 & +1.201 & 174057.00 & -281836.0 & \\
\hline IGR J17456-2901 & $?$ & 1 & & 359.930 & -0.048 & 174538.5 & -290115 & AX J1745.6-2900, Sgr A* \\
\hline V2400 Oph & $\mathrm{CV}$ & 1 & & 359.867 & +8.739 & 171236.45 & -241444.6 & RX J1712.6-2414 \\
\hline XTEJ1817-330 & $\mathrm{LMXB}, \mathrm{T}, \mathrm{BHC}$ & 14 & 6 & 359.817 & -7.996 & 181743.54 & -330107.8 & \\
\hline XTE J1739-285 & LMXB, T, B & 1,15 & 7 & 359.714 & +1.298 & 173953.95 & -282946.8 & \\
\hline GRS 1741.9-2853 & LMXB, T, B & $5,16,17$ & 7 & 359.612 & +0.734 & 174150 & -285242 & AX J1745.0-2855 \\
\hline SAX J1744.7-2916 & $?$ & 18 & & 359.600 & -0.009 & 174442 & -2916.9 & \\
\hline KS 1741-293 & LMXB, T, B & 1 & 7 & 359.584 & -0.087 & 174458 & -2920.2 & \\
\hline 1A $1742-294$ & LMXB, B & 1 & 7,9 & 359.559 & -0.389 & 174605.5 & -293055 & \\
\hline SLX 1744-299/300 & LMXB, B & 1 & 7 & 359.296 & -0.889 & 174725.9 & -295958 & \\
\hline 1E 1740.7-2942 & $\mathrm{LMXB}, \mathrm{BHC}$ & 1 & 6 & 359.116 & -0.106 & 174354.83 & -294442.6 & \\
\hline GRS 1734-292 & AGN, Sy1 & 1 & & 358.844 & +1.395 & 173724.3 & -291048 & GRS $1734-294$ in [1] \\
\hline GRS 1747-312 & $\mathrm{LMXB}, \mathrm{G}, \mathrm{T}, \mathrm{B}$ & 1,19 & & 358.555 & -2.168 & 175045.5 & -311732 & in Terzan 6 \\
\hline IGR J17460-3047 & $?$ & 1 & & 358.494 & -1.094 & 174619 & -3047.5 & Artefact in [26] \\
\hline IGR J17391-3021 & HMXB, NS, Be?, T & 1 & & 358.068 & +0.445 & 173911.58 & -30 2037.6 & XTE J1739-302 \\
\hline IGR J17285-2922 & $\mathrm{BHC} ?, \mathrm{~T}$ & 1 & & 357.630 & +2.923 & 1728.5 & -2922 & \\
\hline $\mathrm{H} 1743-322$ & LMXB, T, BHC & 1 & 6 & 357.255 & -1.833 & 174615.61 & -321359.9 & IGR J17464-3213 \\
\hline IGR J17488-3253 & $?$ & 1 & & 356.962 & -2.662 & 174854.71 & -325444.0 & \\
\hline $3 \mathrm{~A} 1822-371$ & LMXB, XP, D & 1,20 & 10 & 356.850 & -11.291 & 182546.8 & -370619 & \\
\hline SLX 1746-331 & LMXB?, BHC & 5,9 & & 356.807 & -2.973 & 174948.50 & -331218.3 & \\
\hline XTE J1710-281 & LMXB, T, B & 1 & & 356.357 & +6.922 & 171012.3 & -280754 & \\
\hline $4 U 1722-30$ & $\mathrm{LMXB}, \mathrm{G}, \mathrm{B}, \mathrm{A}$ & 1 & 7 & 356.320 & +2.298 & 172733.2 & -304807 & XB 1724-30; in Terzan 2 \\
\hline IGR J17200-3116 & $?, \mathrm{~T}$ & 1 & & 355.022 & +3.346 & 172006.10 & -311702.0 & 1RXS J172006.1-311702 \\
\hline MXB 1730-335 & LMXB, G, B, T & 1 & 7 & 354.841 & -0.158 & 173324.10 & -332316.0 & The Rapid Burster; in Liller 1 \\
\hline XTE J1720-318 & LMXB, T, BHC & 1 & & 354.597 & +3.087 & 171958 & -3146.8 & \\
\hline GX 354-0 & $\mathrm{LMXB}, \mathrm{B}, \mathrm{A}$ & 1 & 7,8 & 354.302 & -0.150 & 173157.4 & -335005 & 4U 1728-337 \\
\hline
\end{tabular}


Table 1. continued.

\begin{tabular}{l|lllcclll}
\hline \hline Source & Type & Ref & Fig. & $1_{\text {II }}$ & b $_{\text {II }}$ & RA & Dec & Comment \\
\hline IGR J17254-3257 & LMXB?, B & 1,21 & & 354.280 & +1.472 & 172525.50 & -325717.5 & 1 RXS J172525.5-325717 \\
1A 1744-361 & LMXB, T, B, A? & 1,22 & & 354.140 & -4.204 & 174819.22 & -360716.6 & XTE J1748-361 \\
1H 1746-370 & LMXB, G, B, A & 1 & & 353.531 & -5.005 & 175012.7 & -370308 & in NGC 6441 \\
XTE J1743-363 & ?,T & 1 & 10 & 353.392 & -3.402 & 174300.0 & -362041 & \\
4U 1705-32 & LMXB, B & 1 & & 352.794 & +4.681 & 170854.40 & -321857.5 & \\
IGR J17252-3616 & HMXB, XP, T & 23,24 & 10 & 351.510 & -0.356 & 172514 & -3616.4 & EXO 1722-363 \\
IGR J17098-3628 & ?,T & 25 & 10,11 & 349.555 & +2.066 & 170948 & -3628.2 & \\
IGR J17091-3624 & BHC? & 1 & & 349.519 & +2.215 & 170906 & -3624.7 & \\
GX 349+2 & LMXB, Z & 1 & 10 & 349.104 & +2.748 & 170544.5 & -362523 & Sco X-2 \\
SAX J1712.6-3739 & LMXB, T, B & 1 & & 348.935 & +0.928 & 171234.00 & -373836.0 & \\
4U 1700-377 & HMXB & 1 & 10,11 & 347.754 & +2.173 & 170356.77 & -375038.9 & \\
GRO J1655-40 & LMXB, T, BH, D & 9 & 6 & 344.982 & +2.456 & 165400.14 & -395044.9 & \\
OAO 1657-415 & HMXB, XP & 1 & 10 & 344.354 & +0.311 & 170047.9 & -414023 & \\
\hline
\end{tabular}

NOTE: The source type and the reference from where this information is taken are given. We further provide the Galactic as well as the J2000.0 equatorial coordinates; these are taken from the SIMBAD Astronomical Database (except for the sources detected by ASCA, i.e., the "AX J" sources, which are taken from Sakano et al. 2002). For some sources we give alternative names frequently used in the literature. Source type classification (after Bird et al. 2006): ? = unknown type, $\mathrm{A}=$ atoll source (neutron star), AGN = active galactic nucleus, $\mathrm{B}=$ burster (neutron star), $\mathrm{Be}=\mathrm{B}$-type emission-line star, $\mathrm{BH}=$ black hole (dynamically confirmed), $\mathrm{BHC}=$ black-hole candidate, $\mathrm{Cluster}=$ cluster of galaxies, $\mathrm{CV}=$ cataclysmic variable, $\mathrm{D}=$ dipping source, $\mathrm{G}=$ globular cluster source, HMXB = high-mass X-ray binary, LMXB = low-mass X-ray binary, Mol cloud = molecular cloud, NS = neutron star, QSO = quasar, SGR = soft gamma-ray repeater, Sy = Seyfert galaxy, Symb $=$ Symbiotic binary, T $=$ transient source, XP = X-ray pulsar (neutron star), Z = Z-type source (neutron star). References: [1] Bird et al. (2006); [2] Lowes et al. (2002); [3] Forman et al. (1976); [4] Levine et al. (2005); [5] Revnivtsev et al. (2004a); [6] Lutovinov et al. (2004); [7] Chakrabarty \& Roche (1997); [8] in 't Zand (2005); [9] McClintock \& Remillard (2006); [10] Reynolds et al. (1999); [11] Grebenev \& Sunyaev (2004b); [12] Grebenev et al. (2005c); [13] Kretschmar et al. (2004); [14] Remillard et al. (2006); [15] Brandt et al. (2005); [16] Cocchi et al. (1999); [17] Muno et al. (2003b); [18] in 't Zand et al. (1997); [19] in 't Zand et al. (2003); [20] Jonker \& van der Klis (2001); [21] Brandt et al. (2006); [22] Bhattacharyya et al. (2006); [23] Zurita Heras et al. (2006); [24] Thompson et al. (2006); [25] Grebenev et al. (2005a); [26] Bird et al. (2004).

respectively). Note that in a few revolutions no monitoring was done, either due to Crab calibration (see Appendix A) or targetof-opportunity observations (revolutions 300, 352, 422-423; MJD 53458-53460, MJD 53613-53616, MJD 53823-53828, respectively). Some single pointings were lost due to other reasons, such as ground station outages. The total net observing time was $727 \mathrm{ks}$.

For each INTEGRAL pointing, the OMC monitors the sources in its field of view by means of shots of variable integration time. Typical values in the range 10 to $200 \mathrm{~s}$ (currently 10 , 50 and $200 \mathrm{~s}$ ) are used to optimize sensitivity, and to minimize read-out noise and cosmic-ray effects. For the faintest objects, several $200 \mathrm{~s}$ exposures in the same pointing can be combined during data analysis on the ground. Telemetry constraints do not allow to download the entire OMC image. For this reason, windows are selected around the proposed X-ray $/ \gamma$-ray targets as well as other targets of interest in the same field of view. Only sub-windows of the CCD with a size of $11 \times 11$ pixels $\left(3.2^{\prime} \times 3.2^{\prime}\right)$ containing those objects are transmitted to ground. For extended sources or sources with poor precision in their coordinates, a mosaic of such sub-windows are set around the source position to cover the entire error circle. The sources in the Galactic bulge region being monitored by the OMC are indicated in Table 2 . Since revolution 421 (March 25, 2006; MJD 53819) onwards, the OMC instrument is operating with a new Input Catalogue (version 0005). Using this catalogue, OMC monitors all sources detected by IBIS/ISGRI (Bird et al. 2006) in the Galactic bulge which fall in its field of view.

The OMC data of the whole period has been processed with an updated OMC Off-line Scientific Analysis software ${ }^{2}$. The full analysis has been working in an automatic way since the third season of the Galactic bulge monitoring, i.e., revolutions 406-429 (February to April 2006; MJD 53775-53846).

\footnotetext{
2 The updated software will be included in OSA 6.0.
}

Table 2. Sources monitored by the OMC in the Galactic bulge region. The ordering is the same as Table 1. Listed are the source name, OMC identifier and the period of monitoring (period I covers the revolutions before 421, period II covers revolution 421 and onwards). Revolution 421 corresponds to 2006, March 25 or MJD 53819. The results for IGR J17544-2619 are shown in Fig. 17.

\begin{tabular}{l|lc}
\hline \hline Source & OMC identifier & Period \\
\hline GRS 1758-258 & IOMC 6846000121 & II \\
IGR J17544-2619 & IOMC 6849000050 & II \\
GX 3+1 & IOMC 6836000093 & II \\
SLX 1735-269 & IOMC 6835000362 & I, II \\
XTE J1748-288 & IOMC 6840000094 & II \\
IGR J17475-2822 & IOMC 6840000103 & II \\
1E 1743.1-2843 & IOMC 6840000034 & I, II \\
SAX J1747.0-2853 & IOMC 6840000039 & II \\
IGR J17407-2808 & IOMC 6839000184 & I, II \\
SLX 1737-282 & IOMC 6839000170 & II \\
IGR J17456-2901 & IOMC 6840000098 & I, II \\
SAX J1744.7-2916 & IOMC 6840000084 & I, II \\
KS 1741-293 & IOMC 6840000085 & I, II \\
1A 1742-294 & IOMC 6840000080 & I, II \\
SLX 1744-299 & IOMC 6840000042 & II \\
1E 1740.7-2942 & IOMC 6840000077 & I, II \\
GRS 1734-292 & IOMC 6839000175 & I, II \\
IGR J17460-3047 & IOMC 7377000086 & II \\
IGR J17391-3021 & IOMC 7376000097 & I, II \\
IGR J17285-2922 & IOMC 6838000560 & I, II \\
H1743-322 & IOMC 7381000052 & II \\
IGR J17488-3253 & IOMC 7381000160 & II \\
SLX 1746-331 & IOMC 7381000150 & II \\
4U 1722-30 & IOMC 7375000273 & I, II \\
\hline
\end{tabular}

Light curves are produced in a short period of time, usually less than $24 \mathrm{~h}$ after the observation is performed. Since the Galactic bulge region is a very crowded field for the OMC, in the flux extraction process we force the photometric aperture to be 

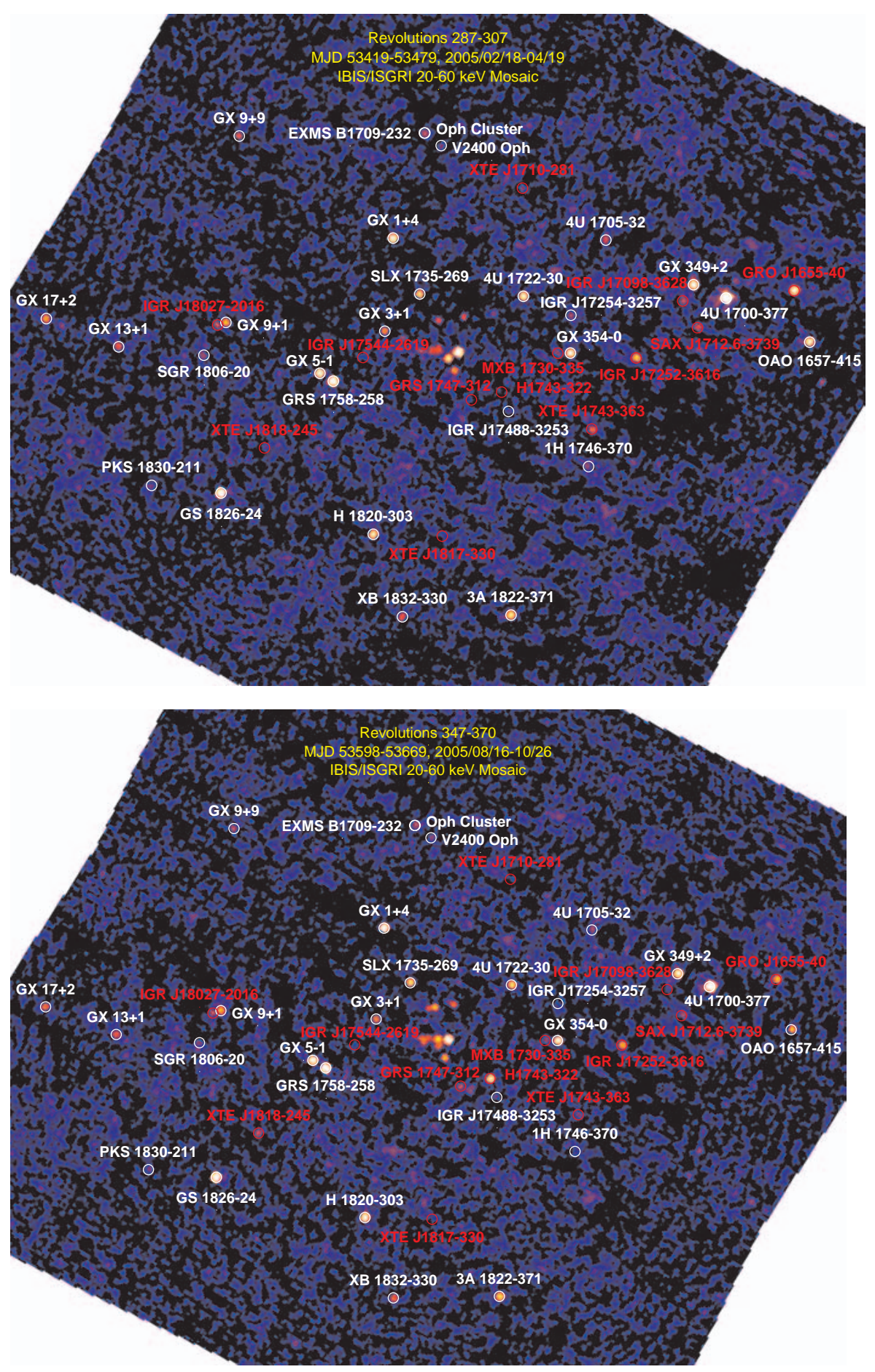

Fig. 1. INTEGRAL IBIS/ISGRI $(20-60 \mathrm{keV})$ mosaic significance images from the first (top; total exposure of $234 \mathrm{ks}$ ) and second season (bottom; total exposure of $235 \mathrm{ks}$ ), using the Hammer-Aitoff projection. Sources and their names are annotated, except for those in the Galactic Center region. The latter are displayed in Fig. 3. Transients (see Table 1) are indicated in red, the other sources in white. The annotated sources are those which reached a significance higher than 7 in either a single revolution, a season or all seasons together (see Table 3 ). centred at the source coordinates, which are taken from the OMC Input Catalogue. This allows us to monitor not only the sources already detected by OMC, but also those previously undetected that could show, for example, bright flares detectable occasionally by OMC. Obviously, having accurate source coordinates is very important to obtain reliable results.

OMC's typical limiting magnitude in the Galactic bulge observations is between $V=15$ and $16 \mathrm{mag}(3 \sigma)$. The actual value depends on sky background and source contamination, which can be very important in this region. We obtain one photometric point per OMC shot for each source. To increase the signal to noise of weak sources, we combine the individual photometric points in the hexagonal dither observation.

\section{Results}

The results we present in this section are organized in two main subsections, one focussing on the seasonal and overall source behaviour of all sources in our sample and another on the source variability.

\subsection{IBIS/ISGRI and JEM-X mosaic images}

We show four IBIS/ISGRI (20-60 keV) mosaic significance images, one for each season and a total one, i.e., of all the pointings together, in Figs. 1 and 2. Since the Galactic Center region (in the middle of the figure) contains a considerable amount of hard 

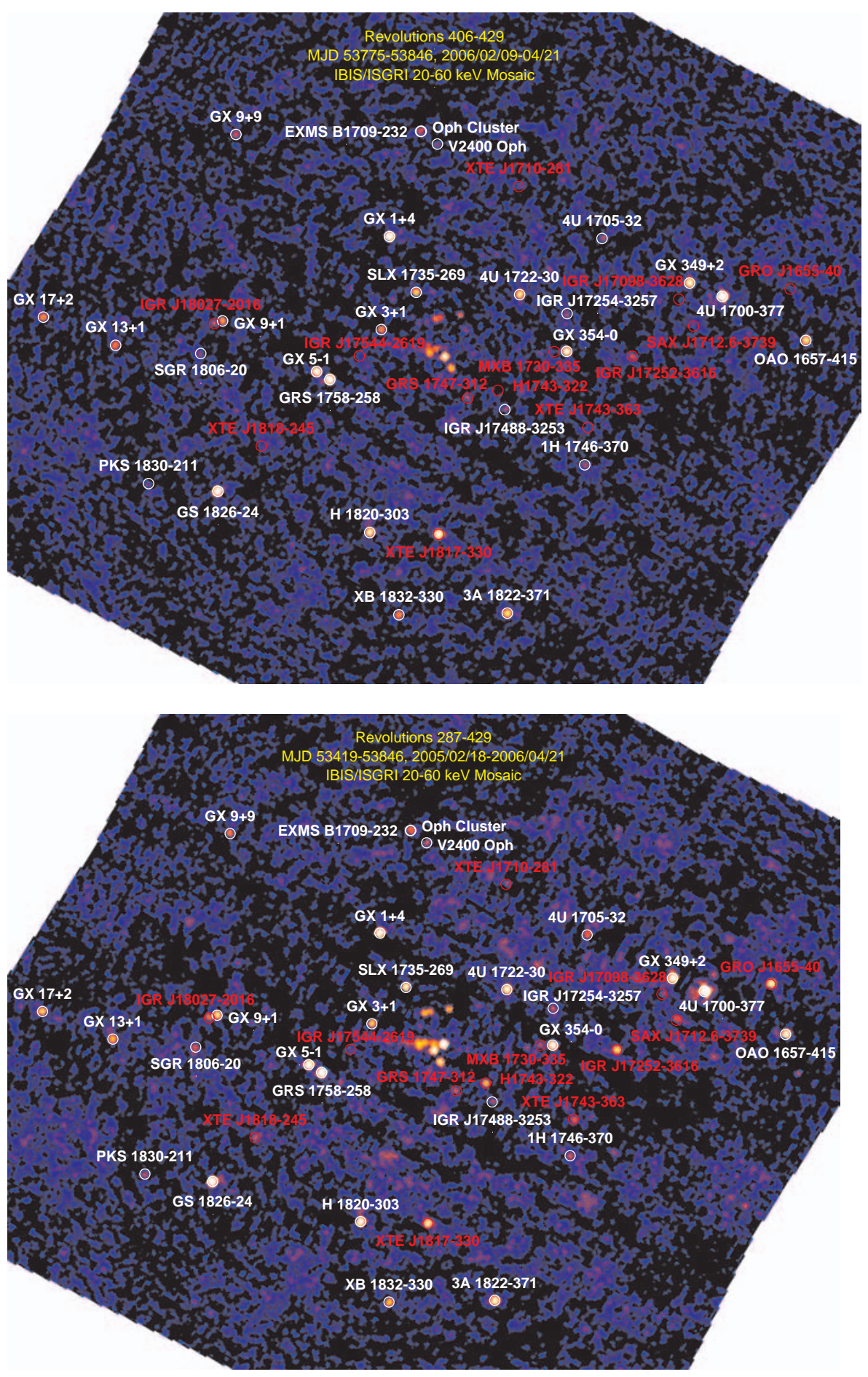

Fig. 2. Same as Fig. 1 but for the third season (top; total exposure of $258 \mathrm{ks}$ ) and all seasons together (bottom; total exposure of $727 \mathrm{ks}$ ).
X-ray sources close together, we zoom in on this region in Fig. 3. Similarly, JEM-X (3-10 keV) significance mosaic images for the three seasons and all the pointings together are shown in Fig. 4. Since annotating all the sources in our sample on the IBIS/ISGRI and JEM-X images would make them unreadable, we chose to label only the sources with a significance of 7 or higher in the $20-60 \mathrm{keV}$ data during either a single hexagonal dither observation, during one season, or during all seasons combined (see Table 3 ). This results in the inclusion of fast transients that only appear within one or a couple of hexagonal dither observations, transients which show outbursts on week to month time scales, and all persistent sources. To avoid crowdedness of source names in the JEM-X images near the Galactic center we did not annotate all sources in that region.

The source detection significances and corresponding average fluxes are given in Tables $3(20-60 \mathrm{keV})$ and 4 $(60-150 \mathrm{keV})$. When a source is not detected we provide $3 \sigma$ upper limits. We additionally give the highest detection significance reached of all the single hexagonal dither observations; these are used to determine whether long-term light curves are shown or not (see Sect. 4.2). In Table 4 we show information only for those sources which were detected above a significance level of 7 in the $60-150 \mathrm{keV}$ band, either in a single hexagonal dither observation, in the average mosaic per season, or in 

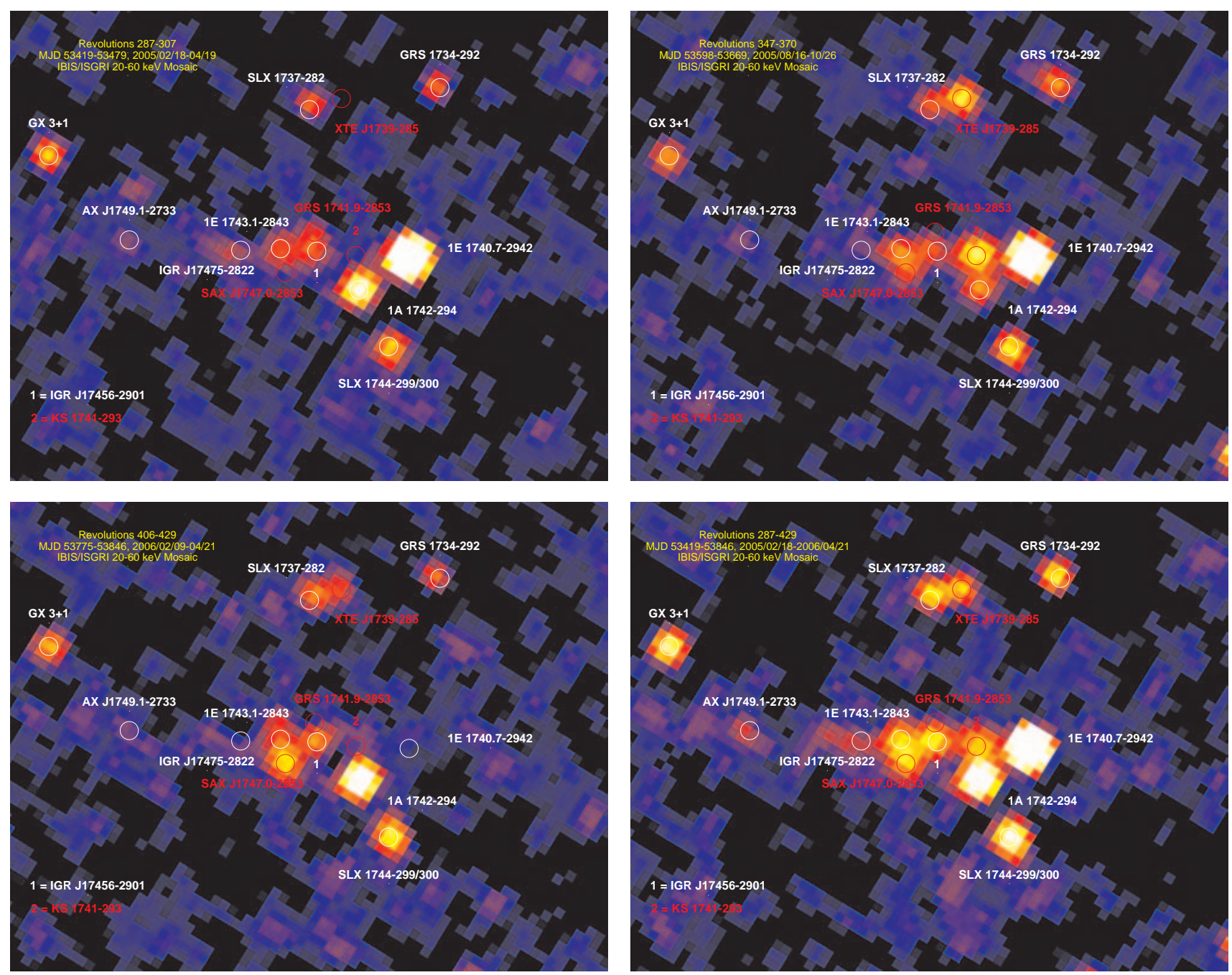

Fig. 3. Same as Figs. 1 and 2, but now zoomed in on the Galactic Center region.

the mosaic of all observations. In Table 5 we give some information for each of the JEM-X mosaic images, such as raw and effective exposure time at the center of the images and which sources are visible above a detection limit of $3 \sigma$. Unfortunately, the current JEM-X analysis software does not allow us to extract accurate flux information from the mosaic images as is the case for IBIS/ISGRI.

Most of the bright well-known persistent and transient $\mathrm{X}$-ray binaries are easily detected in either or all of the four JEM-X and IBIS/ISGRI 20-60 keV mosaic images. Note that 1E 1740.7-2942, normally one of the brightest sources in the Galactic center region, turned off during the whole third season (see Sects. 4.2.1 and 4.3). Some of the fast transients were not detected in the mosaics per season or for the whole period (such as IGR J17544-2619). Some of the weaker sources are detected during every season's average (e.g., IGR J18027-2016, GX 9+9, GRS 1734-292); some are only detected during one or two season averages (e.g., 1E 1743.1-2843, IGR 17456-2901). The latter is also true for some of the transient sources, such as IGR J17475-2822, GRS 1747-312 [in Terzan 6], and SAX J1712.6-3739. We note that IGR 17456-2901 (or a source coincident with it) was detected during the third season when the 1E1740.7-2942 and neighbouring sources were off (see Sect. 4.3). Sources detected only in the average over the whole monitoring period include various kinds of sources, such as PKS 1830-211 (AGN), V2400 Oph (cataclysmic variable), XTE J1710-281 (low-mass X-ray binary).

From Table 4 we see that only about a dozen sources are visible at $60-150 \mathrm{keV}$. Different kinds of sources are detected at these energies, such as 1E 1740.7-2942 (blackhole candidate), GRO J1655-40 (transient black-hole binary), GS 1826-24 (X-ray burster), and 4U 1700-377 (high-mass $\mathrm{X}$-ray binary).

EXMS B1709-232 was detected in the first two seasons, while the OphCluster was only detected in the third season. These two sources are only $1.7^{\prime}$ apart and can, therefore, not be resolved by IBIS/ISGRI. For this reason we attribute the observed flux from only one source which we label Oph Cluster (see also Bird et al. 2006).

Of the weak sources mentioned above, only the persistent sources 1E 1743.1-2843 (low-mass X-ray binary) and GRS 1734-292 (AGN), and the transient low-mass X-ray binary GRS $1747-312$, are detected by JEM-X (see Table 5). GRS 1747-312 was also seen in JEM-X mosaics of our individual hexagonal dither observations near the start of the third season (Chenevez et al. 2006a), consistent with the fact that it shows outbursts roughly every 4.5 months (in 't Zand et al. 2003). It was barely detected in the IBIS/ISGRI mosaic of the individual hexagonal dither observation at the same time (at $6 \sigma$, $\simeq 11 \mathrm{mCrab}, 20-60 \mathrm{keV}$ ). 

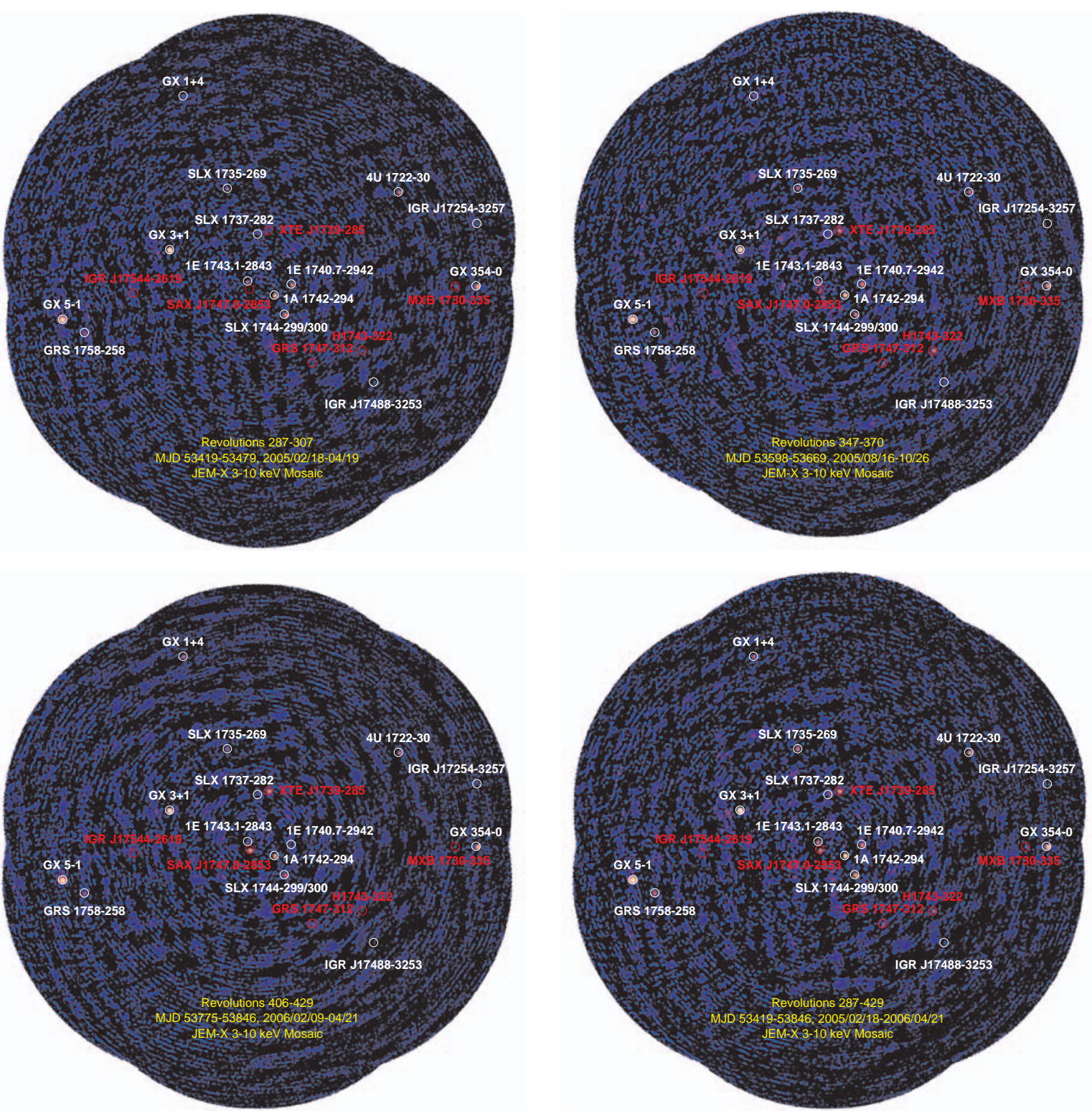

Fig. 4. Same as Figs. 1 and 2, but for JEM-X (3-10 keV). The same sources as in Figs. 1 and 3 are annotated except for AX J1749.1-2733, IGR J17475-2822, IGR J17456-2901, GRS 1741.9-2853, KS 1741-293 and GRS 1734-292 (all in the Galactic Center region) for reason of clarity.

Table 3. This table lists the maximum detection significance reached during the hexagonal dither observations (Sigrev, max $)$, the detection significance per season $\left(\operatorname{Sig}_{\mathrm{sx}}\right.$; where $X=1,2,3$ for the three seasons) and the detection significance for all observations together (Sigall), together with the corresponding fluxes $\left(F_{\mathrm{s}, \mathrm{x}}\right)$ and errors in the fluxes for the IBIS/ISGRI $20-60 \mathrm{keV}$ band. The ordering of sources is the same as used for Table 1. When a source was not detected it is indicated with "-"; we then provide a $3 \sigma$ (statistical) upper limit on the flux. Whenever Sigrev, max exceeds 7, the source long-term light curves are discussed in Sect. 4.2.

\begin{tabular}{|c|c|c|c|c|c|c|c|c|c|}
\hline Source & $\mathrm{Sig}_{\text {rev, } \max }$ & $\mathrm{Sig}_{\mathrm{s} 1}$ & $\begin{array}{c}F_{\mathrm{s} 1} \\
(\mathrm{mCrab})\end{array}$ & $\mathrm{Sig}_{\mathrm{s} 2}$ & $\begin{array}{c}F_{\mathrm{s} 2} \\
(\mathrm{mCrab})\end{array}$ & $\mathrm{Sig}_{\mathrm{s} 3}$ & $\begin{array}{c}F_{\mathrm{s} 3} \\
(\mathrm{mCrab})\end{array}$ & $\mathrm{Sig}_{\text {all }}$ & $\begin{array}{c}F_{\text {all }} \\
(\mathrm{mCrab})\end{array}$ \\
\hline GX 17+2 & 12.2 & 21.2 & $52.4 \pm 2.5$ & 17.7 & $41.5 \pm 2.3$ & 20.1 & $45.7 \pm 2.3$ & $\overline{33.0}$ & $46.3 \pm 1.4$ \\
\hline $18.6-1703$ & & - & $<2.6$ & - & $<2.6$ & - & $<2.6$ & - & $<1.5$ \\
\hline & & 13.2 & $10.4 \pm 0.8$ & 10.9 & $8.5 \pm 0.8$ & 16.7 & $13.4 \pm 0.8$ & 24.0 & $11.0 \pm 0.5$ \\
\hline & & 5.6 & $4.3 \pm$ & 5.6 & 7 & 5.2 & $4.3 \pm$ & 8.6 & 0.4 \\
\hline & & 6.6 & 3.0 & 5.7 & $2.4 \pm$ & 5.1 & $2.4 \pm$ & 9.6 & 0.2 \\
\hline & - & - & & - & $<1$ & - & $<1$. & - & $<0$ \\
\hline & & 9.4 & $4.3 \pm$ & 8.1 & $.7 \pm$ & 7.1 & $3.0 \pm$ & 15.9 & $3.7 \pm 0.2$ \\
\hline & 45. & 136.5 & $72.0 \pm$ & 162.3 & $86.6 \pm 0.5$ & 153.8 & $81.1 \pm$ & 259.2 & $79.9 \pm 0.3$ \\
\hline & 9. & 26.7 & $11.0 \pm$ & 18.9 & $7.9 \pm 0.4$ & 22.5 & $9.1 \pm 0.4$ & 40.7 & $9.8 \pm 0.2$ \\
\hline & 6.4 & 14.2 & $9.8 \pm 0.7$ & 8.7 & $6.1 \pm 0.7$ & 11.2 & $7.9 \pm 0.7$ & 19.8 & $7.9 \pm 0.4$ \\
\hline & - & 5.8 & $2.4 \pm$ & - & $<1.3$ & 5.4 & $2.4 \pm 0.4$ & 6.6 & $1.8 \pm 0.2$ \\
\hline H1745-203 & - & - & $<1.2$ & - & $<1.2$ & - & $<1.2$ & - & $<0.7$ \\
\hline
\end{tabular}


Table 3. continued.

\begin{tabular}{|c|c|c|c|c|c|c|c|c|c|}
\hline Source & $\mathrm{Sig}_{\text {rev, } \max }$ & $\mathrm{Sig}_{\mathrm{s} 1}$ & $\begin{array}{c}F_{\mathrm{s} 1} \\
(\mathrm{mCrab})\end{array}$ & $\mathrm{Sig}_{\mathrm{s} 2}$ & $\begin{array}{c}F_{\mathrm{s} 2} \\
(\mathrm{mCrab})\end{array}$ & $\mathrm{Sig}_{\mathrm{s} 3}$ & $\begin{array}{c}F_{\mathrm{s} 3} \\
(\mathrm{mCrab})\end{array}$ & $\mathrm{Sig}_{\text {all }}$ & $\begin{array}{c}F_{\text {all }} \\
(\mathrm{mCrab})\end{array}$ \\
\hline$\overline{\text { IGR J17597-2201 }}$ & 6.1 & - & $<1.1$ & - & $<1.1$ & 4.9 & $1.8 \pm 0.4$ & - & $<0.6$ \\
\hline XTE J1818-245 & 15.6 & - & $<1.2$ & 10.6 & $4.3 \pm 0.4$ & - & $<1.2$ & 8.8 & $1.8 \pm 0.2$ \\
\hline 1RXS J174607.8-213333 & - & - & $<1.1$ & - & $<1.2$ & - & $<1.1$ & - & $<0.7$ \\
\hline GX 5-1 & 48.7 & 73.9 & $23.2 \pm 0.3$ & 104.9 & $33.5 \pm 0.3$ & 108.6 & $34.8 \pm 0.3$ & 166.8 & $30.5 \pm 0.2$ \\
\hline V1223 Sgr & 4.5 & 5.3 & $10.4 \pm 2.0$ & - & $<5.6$ & 4.5 & $7.9 \pm 1.8$ & 5.8 & $6.7 \pm 1.1$ \\
\hline GRS 1758-258 & 68.5 & 212.6 & $65.2 \pm 0.3$ & 230.2 & $72.6 \pm 0.3$ & 182.4 & $56.1 \pm 0.3$ & 361.7 & $64.6 \pm 0.2$ \\
\hline IGR J17544-2619 & 15.3 & - & $<0.9$ & - & $<0.9$ & - & $<0.9$ & - & $<0.5$ \\
\hline H1820-303 & 50.9 & 48.5 & $20.1 \pm 0.4$ & 70.7 & $29.3 \pm 0.4$ & 54.0 & $22.0 \pm 0.4$ & 100.0 & $23.8 \pm 0.2$ \\
\hline IGR J17331-2406 & - & - & $<1.0$ & - & $<1.0$ & - & $<1.0$ & - & $<0.6$ \\
\hline GX $3+1$ & 7.4 & 22.3 & $6.7 \pm 0.3$ & 18.9 & $5.5 \pm 0.3$ & 20.4 & $6.1 \pm 0.3$ & 35.2 & $6.1 \pm 0.2$ \\
\hline GX $1+4$ & 56.2 & 84.8 & $26.8 \pm 0.3$ & 110.0 & $35.4 \pm 0.3$ & 170.9 & $55.5 \pm 0.3$ & 210.4 & $39.0 \pm 0.2$ \\
\hline XTE J1807-294 & - & - & $<0.9$ & - & $<1.0$ & - & $<1.0$ & - & $<0.6$ \\
\hline AX J1749.2-2725 & - & - & $<0.9$ & 4.9 & $1.2 \pm 0.3$ & - & $<0.9$ & - & $<0.5$ \\
\hline AX J1749.1-2733 & 6.8 & 5.6 & $1.8 \pm 0.3$ & - & $<0.9$ & 3.8 & $1.2 \pm 0.3$ & 7.4 & $1.2 \pm 0.2$ \\
\hline XB 1832-330 & 7.6 & 14.3 & $7.9 \pm 0.5$ & 15.6 & $9.1 \pm 0.5$ & 19.9 & $11.0 \pm 0.5$ & 28.8 & $9.8 \pm 0.3$ \\
\hline SLX 1735-269 & 11.9 & 42.8 & $12.2 \pm 0.3$ & 39.0 & $11.6 \pm 0.3$ & 36.7 & $11.0 \pm 0.3$ & 68.8 & $11.6 \pm 0.2$ \\
\hline XTE J1748-288 & - & - & $<0.9$ & - & $<0.9$ & - & $<0.9$ & - & $<0.5$ \\
\hline IGR J17475-2822 & 5.2 & 9.0 & $2.4 \pm 0.3$ & - & $<0.9$ & 4.5 & $1.2 \pm 0.3$ & 8.8 & $1.2 \pm 0.1$ \\
\hline EXMS B1709-232 & 6.4 & 9.3 & $3.7 \pm 0.4$ & 7.9 & $3.0 \pm 0.4$ & - & $<1.2$ & 15.1 & $3.7 \pm 0.2$ \\
\hline IGR J17507-2856 & - & - & $<0.9$ & - & $<0.8$ & - & $<0.9$ & - & $<0.5$ \\
\hline Oph Cluster & 4.4 & - & $<1.2$ & - & $<1.3$ & 10.2 & $4.3 \pm 0.4$ & - & $<0.7$ \\
\hline IGR J17419-2802 & 5.0 & - & $<0.9$ & - & $<0.9$ & 5.0 & $1.2 \pm 0.2$ & - & $<0.5$ \\
\hline 1E $1743.1-2843$ & 8.0 & - & $<0.9$ & 16.5 & $4.3 \pm 0.2$ & - & $<0.8$ & 24.4 & $4.3 \pm 0.2$ \\
\hline SAX J1747.0-2853 & 15.2 & - & $<0.9$ & - & $<0.8$ & 21.8 & $6.1 \pm 0.3$ & - & $<0.5$ \\
\hline IGR J17407-2808 & 4.6 & - & $<0.9$ & - & $<0.9$ & - & $<0.9$ & - & $<0.5$ \\
\hline SLX 1737-282 & 7.0 & 14.1 & $4.3 \pm 0.3$ & - & $<0.9$ & 15.2 & $4.3 \pm 0.3$ & 23.5 & $3.7 \pm 0.2$ \\
\hline IGR J17456-2901 & 5.9 & - & $<0.9$ & - & $<0.8$ & 14.5 & $4.3 \pm 0.3$ & - & $<0.5$ \\
\hline V2400 Oph & 5.3 & 6.2 & $2.4 \pm 0.4$ & 6.1 & $2.4 \pm 0.4$ & 7.1 & $3.0 \pm 0.4$ & 11.2 & $2.4 \pm 0.2$ \\
\hline XTE J1817-330 & 53.2 & - & $<1.0$ & - & $<1.2$ & 79.6 & $29.9 \pm 0.4$ & 47.7 & $10.4 \pm 0.2$ \\
\hline XTE J1739-285 & 13.7 & - & $<0.9$ & 22.3 & $6.1 \pm 0.3$ & - & $<0.9$ & - & $<0.5$ \\
\hline GRS 1741.9-2853 & 9.6 & - & $<0.9$ & - & $<0.8$ & - & $<0.9$ & 13.9 & $2.4 \pm 0.2$ \\
\hline SAX J1744.7-2916 & 5.1 & - & $<0.9$ & - & $<0.9$ & 4.9 & $1.2 \pm 0.2$ & - & $<0.5$ \\
\hline KS 1741-293 & 19.3 & 5.3 & $1.2 \pm 0.2$ & 28.1 & $7.9 \pm 0.3$ & - & $<0.9$ & 19.7 & $3.0 \pm 0.2$ \\
\hline 1A 1742-294 & 25.5 & 41.5 & $11.6 \pm 0.3$ & 12.6 & $3.7 \pm 0.3$ & 63.1 & $18.3 \pm 0.3$ & 68.0 & $11.0 \pm 0.2$ \\
\hline SLX 1744-299/300 & 9.8 & 20.1 & $6.1 \pm 0.3$ & 26.6 & $7.9 \pm 0.3$ & 26.8 & $7.9 \pm 0.3$ & 42.0 & $7.3 \pm 0.2$ \\
\hline 1E 1740.7-2942 & 47.1 & 162.4 & $45.7 \pm 0.3$ & 139.5 & $39.6 \pm 0.3$ & - & $<0.8$ & 173.1 & $28.7 \pm 0.2$ \\
\hline GRS 1734-292 & 6.6 & 15.3 & $4.3 \pm 0.3$ & 16.9 & $4.9 \pm 0.3$ & 15.5 & $4.3 \pm 0.3$ & 28.0 & $4.9 \pm 0.2$ \\
\hline GRS $1747-312$ & 6.0 & - & $<0.9$ & 9.0 & $2.4 \pm 0.3$ & 9.2 & $2.4 \pm 0.3$ & 11.5 & $1.8 \pm 0.2$ \\
\hline IGR J17460-3047 & - & - & $<0.9$ & - & $<0.9$ & - & $<0.9$ & - & $<0.5$ \\
\hline IGR J17391-3021 & 4.3 & - & $<0.9$ & - & $<0.9$ & - & $<0.9$ & - & $<0.5$ \\
\hline IGR J17285-2922 & 4.0 & - & $<0.9$ & - & $<0.9$ & - & $<0.9$ & - & $<0.5$ \\
\hline H1743-322 & 49.7 & - & $<0.9$ & 50.4 & $15.2 \pm 0.3$ & - & $<0.9$ & 27.9 & $4.9 \pm 0.2$ \\
\hline IGR J17488-3253 & 4.4 & - & $<0.9$ & - & $<0.9$ & 8.5 & $2.4 \pm 0.3$ & 9.4 & $1.8 \pm 0.2$ \\
\hline 3A 1822-371 & 14.1 & 39.3 & $22.6 \pm 0.5$ & 31.8 & $18.3 \pm 0.6$ & 35.1 & $20.1 \pm 0.5$ & 61.8 & $20.1 \pm 0.3$ \\
\hline SLX 1746-331 & - & - & $<0.9$ & - & $<0.9$ & - & $<0.9$ & - & $<0.5$ \\
\hline XTE J1710-281 & 5.6 & 6.5 & $2.4 \pm 0.4$ & - & $<1.2$ & 5.6 & $2.4 \pm 0.4$ & 8.5 & $1.8 \pm 0.2$ \\
\hline 4U 1722-30 & 18.0 & 60.3 & $18.3 \pm 0.3$ & 36.8 & $11.6 \pm 0.3$ & 41.5 & $12.8 \pm 0.3$ & 80.1 & $14.0 \pm 0.2$ \\
\hline IGR J17200-3116 & - & 6.3 & $1.8 \pm 0.3$ & - & $<1.0$ & - & $<1.0$ & 6.6 & $1.2 \pm 0.2$ \\
\hline MXB 1730-335 & 12.2 & 6.5 & $1.8 \pm 0.3$ & 6.4 & $1.8 \pm 0.3$ & - & $<1.0$ & 7.9 & $1.2 \pm 0.2$ \\
\hline XTE J1720-318 & - & - & $<1.0$ & - & $<1.0$ & - & $<1.0$ & - & $<0.6$ \\
\hline GX 354-0 & 48.1 & 69.2 & $22.0 \pm 0.3$ & 71.1 & $23.2 \pm 0.3$ & 83.0 & $26.8 \pm 0.3$ & 128.8 & $24.4 \pm 0.2$ \\
\hline IGR J17254-3257 & - & 6.7 & $1.8 \pm 0.3$ & - & $<1.0$ & 6.2 & $1.8 \pm 0.3$ & 8.0 & $1.2 \pm 0.2$ \\
\hline 1A $1744-361$ & - & - & $<1.1$ & - & $<1.1$ & - & $<1.1$ & - & $<0.6$ \\
\hline 1H 1746-370 & 5.5 & 6.2 & $2.4 \pm 0.4$ & - & $<1.2$ & 6.5 & $2.4 \pm 0.4$ & 9.0 & $1.8 \pm 0.2$ \\
\hline XTE J1743-363 & 9.1 & 14.4 & $5.5 \pm 0.4$ & 6.0 & $2.4 \pm 0.4$ & - & $<1.2$ & 13.1 & $3.0 \pm 0.2$ \\
\hline 4U 1705-32 & 5.1 & 10.3 & $4.3 \pm 0.4$ & 6.8 & $3.0 \pm 0.4$ & 8.0 & $3.0 \pm 0.4$ & 13.7 & $3.0 \pm 0.2$ \\
\hline IGR J17252-3616 & 36.2 & 28.7 & $11.6 \pm 0.4$ & 23.9 & $9.8 \pm 0.4$ & 10.3 & $4.3 \pm 0.4$ & 36.8 & $8.5 \pm 0.2$ \\
\hline IGR J17098-3628 & 23.8 & 8.7 & $4.3 \pm 0.5$ & - & $<1.5$ & - & $<1.5$ & 7.4 & $2.4 \pm 0.3$ \\
\hline IGR J17091-3624 & - & - & $<1.5$ & - & $<1.5$ & - & $<1.5$ & - & $<0.9$ \\
\hline GX 349+2 & 24.5 & 70.7 & $36.6 \pm 0.5$ & 51.7 & $28.0 \pm 0.5$ & 57.9 & $30.5 \pm 0.5$ & 103.3 & $31.7 \pm 0.3$ \\
\hline SAX J1712.6-3739 & 5.6 & 10.1 & $5.5 \pm 0.5$ & 6.4 & $3.7 \pm 0.5$ & - & $<1.6$ & 11.6 & $3.7 \pm 0.3$ \\
\hline 4U 1700-377 & 194.5 & 344.5 & $214.0 \pm 0.6$ & 268.9 & $174.4 \pm 0.7$ & 216.9 & $137.2 \pm 0.6$ & 481.5 & $176.2 \pm 0.4$ \\
\hline GRO J1655-40 & 71.7 & 55.9 & $59.8 \pm 1.0$ & 24.3 & $27.4 \pm 1.2$ & - & $<3.3$ & 47.9 & $29.9 \pm 0.6$ \\
\hline OAO 1657-415 & 26.9 & 62.2 & $87.8 \pm 1.4$ & 27.2 & $39.6 \pm 1.5$ & 42.8 & $58.5 \pm 1.3$ & 75.9 & $62.8 \pm 0.9$ \\
\hline
\end{tabular}


Table 4. This table lists the maximum detection significance reached during the hexagonal dither observations (Sigev, max $)$, the detection significance per season $\left(\operatorname{Sig}_{\mathrm{sX}}\right.$; where $X=1,2,3$ for the three seasons) and the detection significance for all observations together (Sigall), together with the corresponding fluxes $\left(F_{\mathrm{sX}}\right)$ and errors in the fluxes for the IBIS/ISGRI 60-150 keV band. The ordering of sources is the same as used for Table 1. We only list those sources for which either $\mathrm{Sig}_{\mathrm{rev}, \max }, \mathrm{Sig}_{\mathrm{sX}}$ or $\mathrm{Sig}_{\mathrm{all}}$ reached a value higher than 7. When a source was not detected it is indicated with "-"; we then provide a $3 \sigma$ (statistical) upper limit on the flux. Whenever Sig $_{\text {rev,max }}$ exceeds 7, the source long-term light curves are discussed in Sect. 4.2.

\begin{tabular}{l|ccccccccc}
\hline \hline Source & Sig $_{\text {rev, max }}$ & Sig $_{\mathrm{s} 1}$ & $\begin{array}{c}F_{\mathrm{s} 1} \\
(\mathrm{mCrab})\end{array}$ & Sig $_{\mathrm{s} 2}$ & $\begin{array}{c}F_{\mathrm{s} 2} \\
(\mathrm{mCrab})\end{array}$ & Sig $_{\mathrm{s} 3}$ & $\begin{array}{c}F_{\mathrm{s} 3} \\
(\mathrm{mCrab})\end{array}$ & Sig $_{\text {all }}$ & $\begin{array}{c}F_{\text {all }} \\
(\mathrm{mCrab})\end{array}$ \\
\hline GS 1826-24 & 11.0 & 29.5 & $12.2 \pm 0.4$ & 33.7 & $13.4 \pm 0.4$ & 30.2 & $12.2 \pm 0.4$ & 53.6 & $12.8 \pm 0.2$ \\
GRS 1758-258 & 28.9 & 95.3 & $23.8 \pm 0.2$ & 103.4 & $26.2 \pm 0.2$ & 79.5 & $19.5 \pm 0.2$ & 161.3 & $23.2 \pm 0.1$ \\
GX 1+4 & 9.3 & 14.1 & $3.7 \pm 0.2$ & 18.1 & $4.9 \pm 0.2$ & 23.3 & $6.1 \pm 0.2$ & 31.8 & $4.9 \pm 0.1$ \\
XB 1832-330 & 4.3 & 6.2 & $3.0 \pm 0.4$ & 4.1 & $1.8 \pm 0.4$ & 8.0 & $3.7 \pm 0.4$ & 10.2 & $2.4 \pm 0.2$ \\
SLX 1735-269 & 5.2 & 10.0 & $2.4 \pm 0.2$ & 9.8 & $2.4 \pm 0.2$ & 10.0 & $2.4 \pm 0.2$ & 16.7 & $2.4 \pm 0.1$ \\
XTE J1817-330 & 11.1 & - & $<3.4$ & - & $<3.4$ & 22.1 & $6.7 \pm 0.3$ & 12.6 & $2.4 \pm 0.2$ \\
1A 1742-294 & 5.0 & 6.1 & $1.2 \pm 0.2$ & - & $<2.8$ & 9.4 & $2.4 \pm 0.2$ & 7.6 & $1.2 \pm 0.2$ \\
SLX 1744-299/300 & 4.4 & - & $<2.7$ & 6.7 & $1.8 \pm 0.2$ & 5.4 & $1.2 \pm 0.2$ & 8.2 & $1.2 \pm 0.1$ \\
1E 1740.7-2942 & 19.8 & 58.8 & $14.0 \pm 0.2$ & 50.1 & $11.6 \pm 0.2$ & - & $<2.7$ & 61.9 & $8.5 \pm 0.1$ \\
H1743-322 & 13.2 & - & $<2.7$ & 15.4 & $3.7 \pm 0.2$ & - & $<2.8$ & 9.6 & $1.2 \pm 0.1$ \\
4U 1722-30 & 5.4 & 11.7 & $3.0 \pm 0.2$ & 7.1 & $1.8 \pm 0.2$ & 6.9 & $1.8 \pm 0.2$ & 15.2 & $2.4 \pm 0.1$ \\
IGR J17098-3628 & 10.0 & - & $<4.2$ & - & $<4.5$ & - & $<4.3$ & - & $<2.5$ \\
4U 1700-377 & 33.7 & 48.0 & $22.0 \pm 0.4$ & 40.0 & $19.5 \pm 0.5$ & 28.4 & $13.4 \pm 0.5$ & 67.7 & $18.3 \pm 0.2$ \\
GRO J1655-40 & 21.4 & 23.0 & $17.1 \pm 0.7$ & 9.4 & $7.3 \pm 0.8$ & - & $<8.7$ & 18.6 & $7.9 \pm 0.4$ \\
\hline
\end{tabular}

Table 5. Compilation of information for each of the JEM-X mosaic images per season and for the whole program (Fig. 4). We report the season which is refered to, the INTEGRAL revolutions corresponding to the season, the total number of single pointings $(\mathrm{ScWs})$, the total exposure time at the center of each mosaic $\left(t_{\mathrm{exp}}\right)$, the effective exposure time $\left(t_{\text {eff }}\right)$ when taking into account vignetting and other effects inherent to the JEM-X instrument (see Lund et al. 2003), and the list of sources which are detected above the $3 \sigma$ level in the $3-10 \mathrm{keV}$ and $10-25 \mathrm{keV}$ bands during a season or in the average over the whole program. When the detection significance is lower than 3 or a source is not detected we indicate it with (-). The ordering of the sources is the same as in Table 1.

\begin{tabular}{l|cccc}
\hline \hline Season & 1 & 2 & 3 & all \\
Revs & $287-307$ & $347-370$ & $406-429$ & $287-429$ \\
\# ScWs & 132 & 118 & 141 & 391 \\
$t_{\text {exp }}(\mathrm{ks})$ & $\simeq 200$ & $\simeq 180$ & $\simeq 195$ & $\simeq 578$ \\
$t_{\text {eff }}(\mathrm{ks})$ & $\simeq 135$ & $\simeq 122$ & $\simeq 130$ & $\simeq 379$ \\
\hline Source & Detection & significances [3-10]/[10-25] keV \\
\hline GX 5-1 & $150 / 62$ & $174 / 82$ & $158 / 79$ & $276 / 97$ \\
GRS 1758-258 & $8.4 / 14$ & $8.4 / 14$ & $8.5 / 12$ & $14 / 17$ \\
GX 3+1 & $150 / 71$ & $135 / 57$ & $154 / 76$ & $259 / 94$ \\
GX 1+4 & $3.8 / 6.9$ & $7.5 / 10$ & $8.6 / 11.1$ & $11 / 15$ \\
SLX 1735-269 & $8.3 / 9.9$ & $11 / 8.4$ & $7.3 / 15$ & $15 / 11$ \\
1E 1743.1-2843 & $9.0 / 7.7$ & $10 / 8.6$ & $5.6 / 4.1$ & $14 / 9.2$ \\
SAX J1747.0-2853 & $-/-$ & $9.0 /-$ & $23 / 14$ & $16 / 7.4$ \\
SLX 1737-282 & $4.7 / 4.0$ & $-/-$ & $3.9 / 5.1$ & $7.3 / 6.6$ \\
XTE J1739-285 & $-/-$ & $20 / 13$ & $21 / 12$ & $24 / 10$ \\
1A 1742-294 & $28 / 24$ & $39 / 28$ & $25 / 31$ & $57 / 38$ \\
SLX 1744-229/300 & $18 / 12$ & $15 / 11$ & $12 / 10$ & $26 / 15$ \\
1E 1740.7-2942 & $20 / 24$ & $19 / 32$ & $-/-$ & $22 / 29$ \\
GRS 1747-312 & $-/-$ & $7.6 / 4.4$ & $5.7 / 4.0$ & $8.0 / 3.9$ \\
GRS 1734-292 & $-/-$ & $-/-$ & $-/-$ & $4.6 / 5.3$ \\
H1743-322 & $-/-$ & $18 / 8.9$ & $-/-$ & $9.3 / 3.7$ \\
4U 1722-30 & $14 / 13$ & $14 / 11$ & $11 / 7.0$ & $23 / 14$ \\
MXB 1730-335 & $4.4 /-$ & $5.1 / 3.4$ & $-/-$ & $5.7 /-$ \\
GX 354-0 & $44 / 29$ & $32 / 20$ & $41 / 28$ & $72 / 35$ \\
\hline
\end{tabular}

\subsection{Source variability}

To further explore the overall hard X-ray variability of the sources we computed the mean flux over the whole program, as well as the error in the mean and standard deviation, both for the $20-60 \mathrm{keV}$ and the $60-150 \mathrm{keV}$ bands. They are given in Table 6. The mean flux is calculated by averaging the flux values (weighted by their errors) from all the single pointings. This approach allows us to evaluate the source variability that is expressed in the standard deviation given in Table 6. Note that in this case the mean flux is not the same as the average value obtained from the mosaic image of the three seasons together, discussed in Sect. 4.1 and shown in Table 3. They are slightly overestimated with respect to the values obtained from the mosaic image, as discussed in Appendix C.

In general, Table 6 shows that the standard deviation values $(\sigma)$ strongly correlate with the mean fluxes $(\bar{F})$. It is, therefore, more appropriate to evaluate the relative standard deviations $(\sigma / \bar{F})$. In Fig. 5 we plot the relative standard deviation versus the mean flux in the $20-60 \mathrm{keV}$ band $\left(\overline{F_{\mathrm{A}}}\right)$ for those sources which reached a significance higher than 7 in either one or more single hexagonal pointing, or in one season, or in all three seasons together (see Table 3). We grouped the sources among source type: black-hole (candidate) binaries, X-ray bursters, X-ray pulsars, other low-mass X-ray binaries (i.e., those which are not members of the former groups), and other, miscellaneous, sources. The different source types are shown with different symbols. All types of sources span more or less the same variability range. The transient sources are the most variable sources (i.e., $\left.\sigma \overline{F_{\mathrm{A}}} \gtrsim 1\right)$. Most bright $\left(\overline{F_{\mathrm{A}}} \gtrsim\right.$ $20 \mathrm{mCrab}$ ) persistent sources have $\sigma / \overline{F_{\mathrm{A}}}$ values of around 0.5 . Two persistent bright sources are outliers and vary little on long time scales; they have $\sigma \overline{F_{\mathrm{A}}} \simeq 0.15$. These are GRS 1758-258, a black-hole candidate, and GS 1826-24, an X-ray burster.

In the following subsections we describe our results for individual sources in more detail. We focus mainly on light curves in the $20-60 \mathrm{keV}$ band; wherever appropriate we also give information on the $60-150 \mathrm{keV}$ results. A study of the long-term soft $\mathrm{X}$-ray $(<20 \mathrm{keV})$ behaviour can be far better done with, e.g., data from the RXTE/ASM or PCA. In this paper we discuss the longterm JEM-X results for the few sources that are detected by both JEM-X and IBIS/ISGRI during either all or at least most single pointings.

Only sources that exceed a $20-60 \mathrm{keV}$ detection significance of 7 in one or more single hexagonal dither observations are 
Table 6. Mean $(\bar{F})$ observed fluxes in the $20-60 \mathrm{keV}$ (denoted by A) and $60-150 \mathrm{keV}$ (denoted by B) bands, respectively, over the whole AO-3 monitoring program. The error given for $\bar{F}$ is the error in the mean. " $\sigma$ " denotes the standard deviation of the averaged values and gives a measure of the variability of a source. The ordering of sources is the same as used for Table 1 .

\begin{tabular}{|c|c|c|c|c|}
\hline Source & $\begin{array}{l}\overline{F_{\mathrm{A}}} \\
\text { (mCrab }\end{array}$ & ${ }^{\sigma}$ & $\begin{array}{l}\overline{F_{\mathrm{B}}} \\
\text { (mCrab) }\end{array}$ & $\sigma$ \\
\hline GX 17+2 & $55.9 \pm 1.9$ & 32.0 & $44.3 \pm 6.9$ & 42.8 \\
\hline SAX J1818.6-1703 & $11.1 \pm 0.9$ & 13.5 & $29.0 \pm 2.5$ & 31.7 \\
\hline GX 13+1 & $15.8 \pm 0.7$ & 14.1 & $30.7 \pm 2.3$ & 34.8 \\
\hline PKS 1830-211 & $11.1 \pm 0.7$ & 12.1 & $30.0 \pm 2.2$ & 35.4 \\
\hline SGR 1806-20 & $7.1 \pm 0.4$ & 7.0 & $17.8 \pm 1.3$ & 14.6 \\
\hline SAX J1805.5-2031 & $5.2 \pm 0.5$ & 4.8 & $17.0 \pm 1.2$ & 13.6 \\
\hline IGR J18027-2016 & $7.6 \pm 0.4$ & 6.2 & $17.6 \pm 1.3$ & 19.4 \\
\hline GS 1826-24 & $84.2 \pm 0.4$ & 13.3 & $53.5 \pm 1.1$ & 27.0 \\
\hline GX 9+1 & $12.2 \pm 0.3$ & 8.2 & $16.0 \pm 1.2$ & 24.7 \\
\hline GX 9+9 & $11.8 \pm 0.6$ & 11.6 & $24.5 \pm 2.0$ & 27.4 \\
\hline 1RXS J175113.3-201214 & $6.2 \pm 0.4$ & 4.8 & $16.2 \pm 1.3$ & 15.8 \\
\hline H1745-203 & $4.9 \pm 0.5$ & 4.6 & $15.7 \pm 1.3$ & 17.8 \\
\hline IGR J17597-2201 & $5.5 \pm 0.4$ & 4.1 & $14.1 \pm 1.1$ & 14.0 \\
\hline XTE J1818-245 & $8.6 \pm 0.4$ & 10.4 & $18.4 \pm 1.2$ & 17.2 \\
\hline 1RXS J174607.8-213333 & $4.5 \pm 0.4$ & 7.0 & $15.3 \pm 1.2$ & 14.2 \\
\hline GX 5-1 & $34.2 \pm 0.2$ & 21.6 & $13.1 \pm 1.0$ & 10.7 \\
\hline V1223 Sgr & $15.1 \pm 1.7$ & 11.3 & $45.6 \pm 4.9$ & 26.0 \\
\hline GRS 1758-258 & $71.0 \pm 0.2$ & 11.0 & $97.9 \pm 0.7$ & 21.6 \\
\hline IGR J17544-2619 & $4.9 \pm 0.3$ & 6.0 & $11.8 \pm 1.0$ & 10.2 \\
\hline H1820-303 & $26.3 \pm 0.3$ & 15.6 & $17.1 \pm 1.2$ & 18.4 \\
\hline IGR J17331-2406 & $4.4 \pm 0.3$ & 4.0 & $13.4 \pm 1.0$ & 16.6 \\
\hline GX $3+1$ & $7.9 \pm 0.2$ & 4.6 & $11.1 \pm 1.0$ & 9.8 \\
\hline GX $1+4$ & $44.0 \pm 0.2$ & 20.4 & $26.0 \pm 0.8$ & 15.7 \\
\hline XTE J1807-294 & $4.4 \pm 0.3$ & 8.2 & $12.9 \pm 1.0$ & 11.7 \\
\hline AX J1749.2-2725 & $3.6 \pm 0.3$ & 3.1 & $8.6 \pm 1.0$ & 10.4 \\
\hline AX J1749.1-2733 & $4.8 \pm 0.3$ & 4.7 & $10.0 \pm 1.1$ & 24.0 \\
\hline XB 1832-330 & $11.8 \pm 0.5$ & 9.0 & $25.9 \pm 1.5$ & 22.4 \\
\hline SLX 1735-269 & $12.7 \pm 0.2$ & 5.5 & $15.7 \pm 0.8$ & 17.2 \\
\hline XTE J1748-288 & $4.1 \pm 0.3$ & 5.4 & $9.7 \pm 1.0$ & 12.9 \\
\hline IGR J17475-2822 & $4.5 \pm 0.3$ & 3.9 & $11.3 \pm 1.0$ & 11.4 \\
\hline EXMS B1709-232 & $7.3 \pm 0.4$ & 5.5 & $17.7 \pm 1.4$ & 15.8 \\
\hline IGR J17507-2856 & $4.2 \pm 0.3$ & 3.5 & $11.1 \pm 1.0$ & 9.0 \\
\hline Oph Cluster & $5.4 \pm 0.5$ & 4.2 & $10.3 \pm 2.0$ & 10.3 \\
\hline IGR J17419-2802 & $4.2 \pm 0.3$ & 3.9 & $12.0 \pm 1.0$ & 10.3 \\
\hline 1E 1743.1 & $5.1 \pm 0.3$ & 4.4 & $9.9 \pm 1.0$ & 11.2 \\
\hline SAX J1747.0-2853 & $7.5 \pm 0.3$ & 6.8 & $10.3 \pm 0.9$ & 11.8 \\
\hline IGR J17407-2808 & $4.2 \pm 0.3$ & 3.8 & $10.0 \pm 1.0$ & 10.6 \\
\hline SLX 1737-282 & $5.4 \pm 0.3$ & 4.1 & $10.7 \pm 1.0$ & 10.8 \\
\hline IGR J17456-2901 & $5.4 \pm 0.4$ & 4.5 & $7.0 \pm 1.4$ & 8.6 \\
\hline V2400 Oph & $6.7 \pm 0.4$ & 4.9 & $15.1 \pm 1.2$ & 12.8 \\
\hline XTE J1817-330 & $35.2 \pm 0.5$ & 25.7 & $34.9 \pm 1.6$ & 21.5 \\
\hline XTE J1739-285 & $7.9 \pm 0.3$ & 6.2 & $12.2 \pm 1.0$ & 13.0 \\
\hline GRS 1741.9-2853 & $4.7 \pm 0.3$ & 5.6 & $10.4 \pm 1.0$ & 10.0 \\
\hline SAX J1744.7-2916 & $4.3 \pm 0.4$ & 5.0 & $10.7 \pm 1.2$ & 12.5 \\
\hline KS 1741-293 & $7.9 \pm 0.3$ & 9.7 & $12.3 \pm 1.0$ & 19.4 \\
\hline 1A 1742-294 & $15.7 \pm 0.2$ & 11.5 & $14.8 \pm 0.8$ & 12.9 \\
\hline SLX 1744-299/300 & $8.6 \pm 0.2$ & 5.0 & $13.7 \pm 0.9$ & 12.4 \\
\hline 1E 1740.7-2942 & $38.8 \pm 0.2$ & 20.2 & $46.9 \pm 0.7$ & 26.3 \\
\hline GRS 1734-292 & $6.2 \pm 0.2$ & 4.4 & $12.7 \pm 0.9$ & 17.0 \\
\hline IGR J17460-3047 & $3.6 \pm 0.3$ & 3.5 & $10.9 \pm 1.0$ & 9.2 \\
\hline GRS 1747-312 & $5.3 \pm 0.5$ & 3.8 & $12.9 \pm 1.5$ & 9.8 \\
\hline IGR J17391-3021 & $4.3 \pm 0.3$ & 5.3 & $11.2 \pm 1.0$ & 11.4 \\
\hline IGR J17285-2922 & $4.1 \pm 0.3$ & 3.2 & $12.7 \pm 1.0$ & 10.2 \\
\hline H1732-322 & $12.8 \pm 0.3$ & 20.2 & $17.1 \pm 0.9$ & 19.2 \\
\hline IGR J17488-3253 & $4.4 \pm 0.3$ & 3.6 & $12.9 \pm 0.9$ & 10.8 \\
\hline 3A 1822-371 & $22.3 \pm 0.4$ & 10.5 & $20.1 \pm 1.6$ & 17.9 \\
\hline SLX 1746-331 & $4.6 \pm 0.3$ & 4.1 & $13.5 \pm 0.9$ & 10.9 \\
\hline XTEJ1710-281 & $6.0 \pm 0.4$ & 8.5 & $14.9 \pm 1.2$ & 17.1 \\
\hline 4U 1722-30 & $16.3 \pm 0.2$ & 6.8 & $15.9 \pm 0.8$ & 10.5 \\
\hline IGR J17200-3116 & $4.4 \pm 0.4$ & 4.7 & $12.0 \pm 1.3$ & 14.0 \\
\hline
\end{tabular}

Table 6. continued.

\begin{tabular}{l|cccc}
\hline \hline & $\begin{array}{c}\overline{F_{\mathrm{A}}} \\
(\mathrm{mCrab})\end{array}$ & \multicolumn{2}{c}{$\begin{array}{c}\overline{F_{\mathrm{B}}} \\
(\mathrm{mCrab})\end{array}$} \\
Source & $5.7 \pm 0.3$ & 5.5 & $14.2 \pm 1.0$ & 15.0 \\
\hline MXB 1730-335 & $4.4 \pm 0.4$ & 4.2 & $12.6 \pm 1.0$ & 10.2 \\
XTE J1720-318 & $27.0 \pm 0.2$ & 18.4 & $14.5 \pm 1.0$ & 12.3 \\
GX 354-0 & $4.5 \pm 0.4$ & 3.7 & $11.7 \pm 1.2$ & 12.7 \\
IGR J17254-3257 & $5.0 \pm 0.4$ & 3.9 & $12.9 \pm 1.2$ & 11.5 \\
1A 1744-361 & $6.3 \pm 0.4$ & 4.6 & $14.3 \pm 1.3$ & 26.4 \\
1H 1746-370 & $6.9 \pm 0.3$ & 6.3 & $14.1 \pm 1.2$ & 21.9 \\
XTE J1743-363 & $6.7 \pm 0.4$ & 5.6 & $16.4 \pm 1.2$ & 12.8 \\
4U 1705-32 & $13.1 \pm 0.3$ & 12.6 & $16.6 \pm 1.2$ & 12.5 \\
IGR J17252-3616 & $7.7 \pm 0.5$ & 11.3 & $16.7 \pm 1.6$ & 19.9 \\
IGR J17098-3628 & $4.8 \pm 0.5$ & 5.4 & $15.1 \pm 1.5$ & 19.9 \\
IGR J17091-3624 & $34.8 \pm 0.4$ & 17.4 & $17.8 \pm 1.7$ & 17.1 \\
GX 349+2 & $8.5 \pm 0.5$ & 7.3 & $21.0 \pm 1.5$ & 21.1 \\
SAX J1712.6-3739 & $191.9 \pm 0.5$ & 197.8 & $91.0 \pm 1.4$ & 84.1 \\
4U 1700-377 & $50.2 \pm 1.0$ & 72.8 & $70.9 \pm 2.6$ & 79.4 \\
GRO J1655-40 & $75.5 \pm 1.1$ & 49.4 & $47.0 \pm 3.5$ & 45.1 \\
OAO 1657-415 & \multicolumn{4}{c}{}
\end{tabular}

considered here. This level was chosen in order to assure that the long-term light curves reveal significant variations. Note that the light curves display all data points, including those where the detection significance was lower than 7 . For most of the sources we show the mean intensities averaged per hexagonal dither observation. Only when sources are highly variable within an observation are the results from the single pointings displayed.

We again grouped the $20-60 \mathrm{keV}$ light-curve figures and results according to source type, i.e., black-hole (candidate) binaries, X-ray bursters, X-ray pulsars, other low-mass X-ray binaries, and miscellaneous sources. To each group of sources we devote a separate subsection. Wherever possible, a comparison to previous hard X-ray monitoring results is done; these comprise mainly observations made by MIT/OSO 7 (1971-1974, Markert et al. 1979), GRANAT/SIGMA (1989-1998, see, e.g., Churazov et al. 1994; Revnivtsev et al. 2004b) and CGRO/BATSE (1991-2000, see, e.g., Harmon et al. 2004).

\subsubsection{Black-hole (candidate) binaries}

1E 1740.7-2942. Normally, the most dominant source within the few degrees of the Galactic Center is the black-hole candidate 1E 1740.7-2942. It is therefore not surprising that earlier hard X-ray $/ \gamma$-ray $(\gtrsim 20 \mathrm{keV})$ measurements of the region could only focus on this source, given the poor spatial resolution of the instruments (see, e.g., Cook et al. 1991; Bazzano et al. 1992; Churazov et al. 1994). Variability in the hard X-ray flux of 1E 1740.7-2942 was already evident from observations with different experiments (see, e.g., Bazzano et al. 1992). In 2005 1E 1740.7-2942 slowly varied on a monthly time scale between $35-65 \mathrm{mCrab}$ and $30-90 \mathrm{mCrab}$, in the 20-60 keV and 60-150 keV bands, respectively (Fig. 6). Similar flux levels were observed previously by GRANAT/SIGMA (see Mandrou et al. 1994; Churazov et al. 1994) and INTEGRAL/IBIS (Del Santo et al. 2005). In spring 2006 (MJD 53775-53846) the source went below the detection limits of JEM-X $(\$ 4 \mathrm{mCrab}$, $3-25 \mathrm{keV}$ ) and IBIS/ISGRI ( $\$ 1 \mathrm{mCrab}, 20-60 \mathrm{keV}$; (see Fig. 6, Table 3; see also Sect. 4.3). Similar "switch-offs" at hard X-rays occurred in 1991/1992 (e.g., Mandrou et al. 1994; Harmon et al. 2004), 1994/1995 (Harmon et al. 2004) and 2004 (Grebenev et al. 2004b; Del Santo et al. 2005). They seem to occur every $\sim 600$ days (Smith et al. 2002). 
GRS 1758-258. This persistent black-hole candidate, located $\simeq 40^{\prime}$ away from GX5-1 (Sect. 4.2.3) varies between $\simeq 60-95 \mathrm{mCrab}$ and $\simeq 60-140 \mathrm{mCrab}$ in the $20-60 \mathrm{keV}$ and $60-150 \mathrm{keV}$ energy bands, respectively, on weekly to monthly time scales. The source is more variable at higher energies, especially during the third season (Fig. 6). The count rates in the two energy bands show that the source is harder than the Crab. Previous INTEGRAL observations (Pottschmidt et al. 2006), as well as observations with GRANAT/SIGMA (Gilfanov et al. 1993; Mandrou et al. 1994; Kuznetsov et al. 1999) and RXTE and CGRO/BATSE (Smith et al. 2001, 2002) showed the source had similar variability, disappearing at various times below the detection limits during a whole season, indicating variability by more than a factor of 10 . Note that this is similar to that seen in 1E 1740.7-2942, as described above.

H1743-322. In 2003 an outburst of this system was detected by INTEGRAL (Revnivtsev et al. 2003) and it was designated IGR J17464-3213. The source was soon associated with H1743-322 (Markwardt \& Swank 2003a,b), from which outbursts had been previously seen (see Kalemci et al. 2006, and references therein). INTEGRAL performed various observations throughout that outburst (Parmar et al. 2003; Lutovinov et al. 2005; Capitanio et al. 2005; Joinet et al. 2005). The source reappeared again in 2004 (Swank 2004) and 2005 (on MJD 53588; Swank et al. 2005). Our program monitored the source from just after the 2005 maximum (Kretschmar et al. 2005; see Fig. 6). The source was clearly seen in both the $20-60 \mathrm{keV}$ and $60-150 \mathrm{keV}$ bands. After a decay with an efolding time scale of $\sim 16$ days, the source rebrightened to $\simeq 70 \mathrm{mCrab}$, after which it decayed with a shorter e-folding time, i.e., $\sim 5$ days $(20-60 \mathrm{keV})$.

XTE J1817-330. A new bright X-ray transient and blackhole candidate was reported in January 2006, designated XTE J1817-330 (Remillard et al. 2006b). INTEGRAL detected it at the start of the third season (see Shaw et al. 2006; Kuulkers et al. 2006a; Goldoni et al. 2006), and showed the source to be quite variable, up to $\simeq 120 \mathrm{mCrab}$ (see Fig. 6). The hard X-ray spectral shapes were also seen to vary substantially, especially near the beginning of the season (Kuulkers et al. 2006a). The transient was active during the whole third season.

GROJ1655-40. Precisely at the start of our program the black-hole X-ray transient GRO J1655-40 was reported to become active (on MJD 53419; Markwardt \& Swank 2005). Our INTEGRAL GRO J1655-40 light curves (Kuulkers et al. 2005a; Shaw et al. 2005c; Fig. 6) nicely complement observations at soft X-ray (RXTE/PCA; see Homan 2005; Shaposhnikov et al. 2006), optical/IR (Buxton et al. 2005; Shaposhnikov et al. 2006) and radio (VLA; see Rupen et al. 2005; Shaposhnikov et al. 2006) wavelengths. The $20-60 \mathrm{keV}$ and $60-150 \mathrm{keV}$ fluxes peaked at $\simeq 400$ and $\simeq 350 \mathrm{mCrab}$, respectively. Contemporaneous hard X-ray coverage was also provided by RXTE/HEXTE (see Homan 2005; Shaposhnikov et al. 2006) and Swift/BAT (Brocksopp et al. 2006). The source was still active during our second season; it is known for its multiple rebrightenings after the main one (see, e.g., Harmon et al. 2004).

\subsubsection{X-ray bursters}

$G X 17+2$. GX $17+2$ is a bright persistent (soft) X-ray source and highly variable on various time scales, one of the characteristics of Z-sources (e.g., Hasinger \& van der Klis 1989). Previous IBIS/ISGRI observations showed the hard X-ray flux (22-40 keV) already to be variable on a $\simeq 10$-day time scale between $\simeq 25-85 \mathrm{mCrab}$ (Paizis et al. 2006). Note that it is

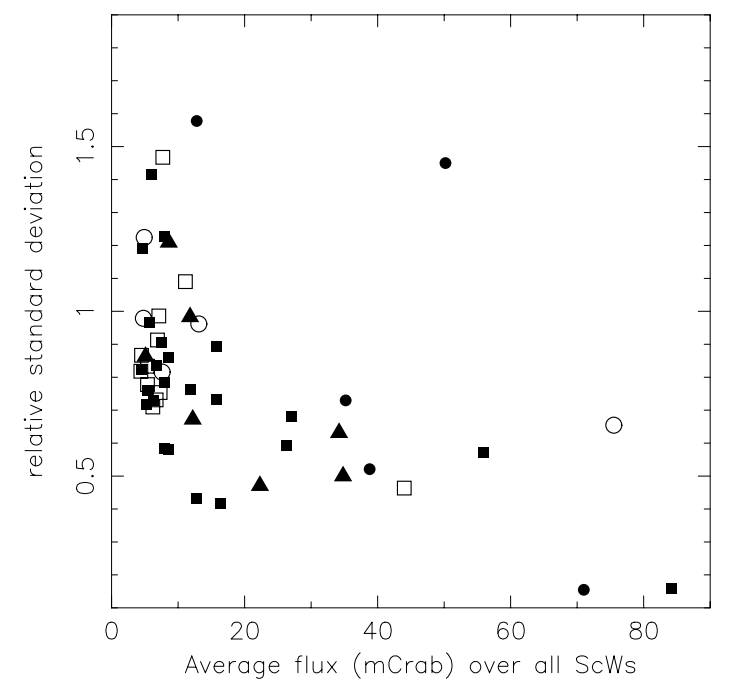

Fig. 5. Relative standard deviation $\left(\sigma / \overline{F_{\mathrm{A}}}\right)$ versus mean flux $\left(\overline{F_{\mathrm{A}}}\right)$ for the $20-60 \mathrm{keV}$ band. The different symbols refer to the different kinds of sources as outlined in Table 1; filled circles: black-hole (candidate) binaries, filled squares: X-ray bursters, filled triangles: other low-mass $\mathrm{X}$-ray binaries, open circles: X-ray pulsars, open squares: miscellaneous sources. $4 \mathrm{U} 1700-377$ is outside the boundaries of the plot.

on average brighter at hard X-rays than the Z-source GX 5-1 (Sect. 4.2.3), whereas at soft X-rays GX 5-1 is the brightest of the "GX"-sources (see, e.g., Hasinger \& van der Klis 1989). GX $17+2$ can flare up to $\simeq 110$ mCrab (20-60 keV, Fig. 7). Similar flaring activity can be discerned from previous observations with IBIS/ISGRI (Piraino et al. 2004). Similarly, the MIT/OSO-7 observations showed variability on a time scale of months from their detection limits up to $\sim 300 \mathrm{mCrab}$ (15-40 keV; Markert et al. 1979).

GX 354-0. GX 354-0 (or 4U 1728-34) is generally seen with a $20-60 \mathrm{keV}$ flux between $10-30 \mathrm{mCrab}$ in our data (Fig. 7). However, every now and then we see it flaring up to $70-80 \mathrm{mCrab}(20-60 \mathrm{keV})$ for a time scale of about a week (Fig. 7). Comparable flux variations on weekly time scales have been observed earlier by INTEGRAL/IBIS (Bazzano et al. 2004, 20-40 keV; Falanga et al. 2006, 20-100 keV), as well as by GRANAT/SIGMA (Claret et al. 1994; see also Mandrou et al. 1994). Similar variability is also present in the $C G R O / \mathrm{BATSE}$ light curves (Harmon et al. 2004; see also Barret et al. 1996). Flux increases up to $200 \mathrm{mCrab}(20-60 \mathrm{keV})$ have been reported during previous INTEGRAL/IBIS observations (Zurita et al. 2004). The MIT/OSO-7 (15-40 keV) observations show that similar flux levels were reached in the early seventies (Markert et al. 1979).

GX 354-0 is detectable in both JEM-X and IBIS/ISGRI most of the time (Fig. 8). Also in the $3-10$ and $10-25 \mathrm{keV}$ band does the flux vary on typically weekly time scales, between $\simeq 60-300 \mathrm{mCrab}$ and $20-150 \mathrm{mCrab}$, respectively. The fluxes in these two bands are clearly correlated. There appears, however, an anti-correlation between the soft (3-10 keV) and hard X-ray $(20-60 \mathrm{keV})$ flux: the highest soft X-ray fluxes are accompanied by low hard X-ray fluxes, while the highest hard Xray fluxes are accompanied by low soft X-ray fluxes. However, at times both the soft and hard X-ray fluxes can be simultaneously low. Similar behaviour can be discerned from the $3-20 \mathrm{keV}$ and 20-100 keV INTEGRAL/JEM-X and IBIS/ISGRI light curves presented by Falanga et al. (2006). We saw 13 type I X-ray bursts spread over our whole program. They occur at different 

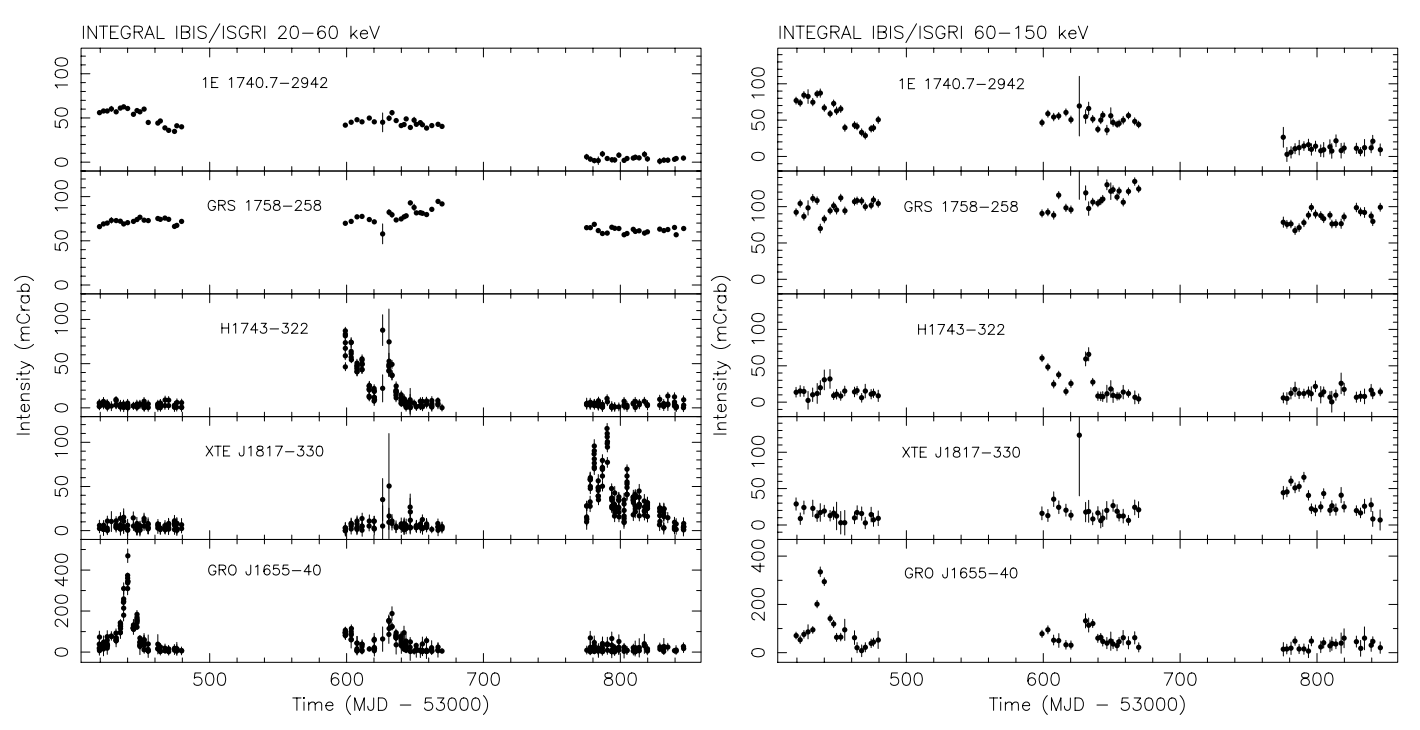

Fig. 6. INTEGRAL IBIS/ISGRI light curves from the three seasons of the Galactic bulge monitoring program, for the energy ranges $20-60 \mathrm{keV}$ (left) and 60-150 keV (right). Shown are the black-hole (candidate) binaries detected during our program: 1E 1740.7-2942, GRS 1758-258, H1743-322, XTEJ1817-330, and GROJ1655-40. In the 20-60 keV band, we show for the first two sources the averages per hexagonal dither observation ( 7 pointings), while for the latter we show single pointing fluxes. The $60-150 \mathrm{keV}$ data points are averages per hexagonal dither observation.

IBIS/ISGRI flux levels, similar to that found by Falanga et al. (2006).

H1820-303 (in NGC 6624). The source generally varies slowly between $10-30 \mathrm{mCrab}$ in the $20-60 \mathrm{keV}$ band (Fig. 7; see also INTEGRAL/IBIS observations presented by Bazzano et al. 2004 in the $20-40 \mathrm{keV}$ band, and Tarana et al. 2006a in the $20-30 \mathrm{keV}, 30-60 \mathrm{keV}$ and $60-120 \mathrm{keV}$ bands). This is consistent with the $20-100 \mathrm{keV}$ upper limits derived from CGRO/BATSE ( $80 \mathrm{mCrab}$ and $30 \mathrm{mCrab}$ for 1-day and 10-day integrations, respectively; Bloser et al. 1996). At the start of our second season (August 2005) the source was bright, i.e., $\simeq 100 \mathrm{mCrab}(20-60 \mathrm{keV})$ and rather hard $(\simeq 40 \mathrm{mCrab}$ in the $60-150 \mathrm{keV}$ band). It declined within 2 weeks to its normal flux level. The same hard state was recently reported by Tarana et al. (2006a) from other INTEGRAL data taken close in time to our monitoring observations. This can be connected to the soft ( $\$ 20 \mathrm{keV})$ low-intensity states which occur roughly every 170 days (e.g., Chou \& Grindlay 2001). Some evidence for variations on time scales of months can be seen in the MIT/OSO-7 observations too, with $15-40 \mathrm{keV}$ fluxes generally being below $100 \mathrm{mCrab}$ (Markert et al. 1979).

GS 1826-24. During the first years of CGRO/BATSE (Harmon et al. 2004) GS 1826-24 was below/near its detection limits; later on it gradually became brighter, reaching up to $\simeq 70 \mathrm{mCrab}$ near the end of the mission $(20-100 \mathrm{keV}$; Harmon et al. 2004). At the moment, the source is one of the brightest persistent type I X-ray bursters in the Galactic bulge region. During our observations, it slowly varies on monthly and longer time scales between $\simeq 70$ and $\simeq 100 \mathrm{mCrab}$ at $20-60 \mathrm{keV}$ (Fig. 7). In the $60-150 \mathrm{keV}$ band, however, the source varies more on a weekly time scale between $\simeq 30$ and $\simeq 80 \mathrm{mCrab}$, around an average flux of $\simeq 55 \mathrm{mCrab}$ (Fig. 11).

KS 1741-293. KS 1741-293 was most of the time not significantly detected during our monitoring observations. However, it was bright, reaching up to $\simeq 25 \mathrm{mCrab}(20-60 \mathrm{keV})$, for about a month during the first half of the second season (August/September 2005, MJD 53599-53633; Fig. 7). KS 1741-293 was earlier seen to be active in March 2003 and March 2004 (Bélanger et al. 2004; Grebenev et al. 2004b;
De Cesare et al. 2006). Type I X-ray bursts were previously found with JEM-X (De Cesare et al. 2006); in our program we did not see any.

MXB 1730-335 (in Liller 1). We saw MXB 1730-335 (better known as The Rapid Burster) turning on at the end of the first season (mid-April 2005; see also Molkov et al. 2005b) and it was turning off at the beginning of the second season (mid-August 2005; Fig. 7; see also Kretschmar et al. 2005). Previous outbursts of MXB 1730-335 were already recorded by IBIS/ISGRI, in February (Falanga et al. 2004) and August 2003 (Sunyaev et al. 2003a). This is consistent with the outburst recurrence period being roughly 100 days since 2000 (Masetti 2002). Strong burst activity is seen in our data near the end of the hard X-ray outburst, between 2005 August 25 and September 3 (MJD 53607-53616).

XTE J1739-285. In August 2005 (second season), the X-ray transient XTE J1739-285 (discovered in 1999, Markwardt et al. 1999) was found by INTEGRAL to be bright at soft and not detected at hard X-ray energies (Bodghee et al. 2005). About a month later the situation had reversed; it was bright at hard and weak at soft X-ray energies (Shaw et al. 2005b). Although at first we attributed the state change to the compact object being a black hole, we proved it to be a neutron star based on the occurrence of type I X-ray bursts detected with JEM-X (Brandt et al. 2005). During the third season the source was still active (Chenevez et al. 2006a, Fig. 7). We saw a total of 13 type I X-ray bursts when the source was active.

SAX J1747.0-2853. SAX J1747.0-2853 is active at relatively low flux levels for long periods (e.g., Wijnands et al. 2002; Natalucci et al. 2004). True quiescence, however, is reached sometimes (Werner et al. 2004). The source was active (Fig. 7) during the end of the second visibility season (October 2005; see Kuulkers et al. 2005c), as well during most part of the third visibility season (e.g., Chenevez et al. 2006a). It reached a maximum of about $24 \mathrm{mCrab}(20-60 \mathrm{keV})$ during the third season. Previous activity, as seen by INTEGRAL, was reported during March 2004 (Deluit et al. 2004). In our data SAX J1747.0-2853 showed 6 type I X-ray bursts, 1 during both the first and second season, the rest during the third season (see also Kuulkers et al. 2005c; Chenevez et al. 2006a). 

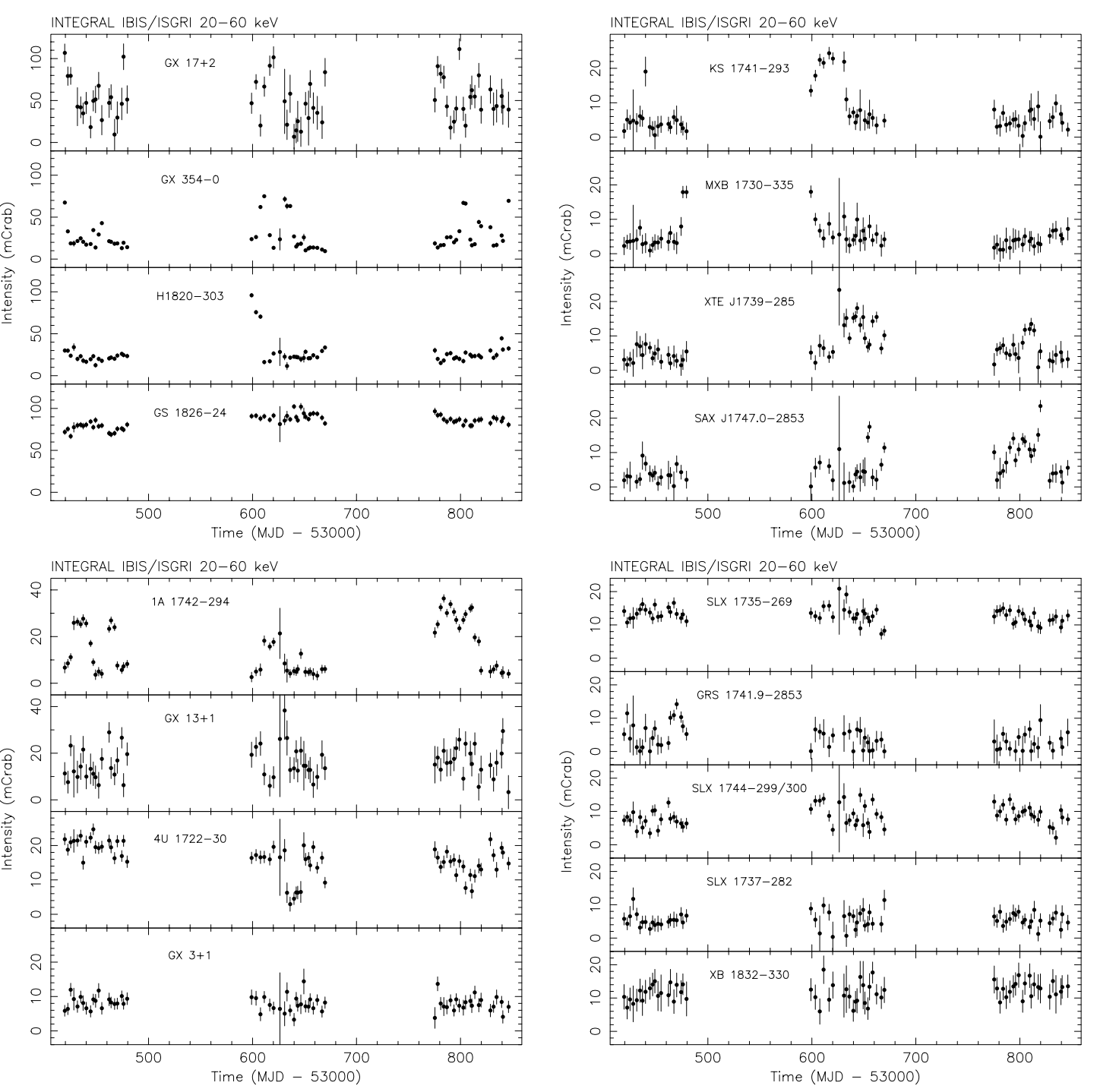

Fig. 7. INTEGRAL IBIS/ISGRI light curves $(20-60 \mathrm{keV})$ from the three seasons of the Galactic bulge monitoring program. Shown are the averages per hexagonal dither observation for the following type I X-ray burst sources: upper left: GX 17+2, GX 354-0, H1820-303, and GS 1826-24; upper right: KS 1741-293, MXB 1730-335, XTEJ1739-285, and SAX J1747.0-2853; lower left: 1A 1742-294, GX 13+1, 4U 1722-30, and GX 3+1; lower right: SLX 1735-269, GRS 1741.9-2853, SLX 1744-299/300, SLX 1737-282, and XB 1832-330.

1A 1742-294. This X-ray burster is $32^{\prime}$ away from 1E 1740.7-2942 and well resolved by INTEGRAL/IBIS (see, e.g., Bélanger et al. 2006). We see it clearly varying up to $\simeq 40 \mathrm{mCrab}(20-60 \mathrm{keV})$ on a monthly time scale (Fig. 7). Similar variability on roughly a half year time scale was earlier reported by Churazov et al. (1995) using GRANAT/SIGMA. This source is the most active type I X-ray burster in our program. We found 36 type I X-ray bursts; most of the bursts were seen when the source was at the highest IBIS/ISGRI flux levels.

Due to the fact that at hard X-ray energies IBIS/ISGRI is able to distinguish the source from neighboring sources, and the simultaneity of the softer X-ray JEM-X information, we are able to study this behaviour for the first time clearly in this source. During most of the pointings we detect 1A 1742-294 with JEM-X (Fig. 9). The source shows the same behaviour between $3-10 \mathrm{keV}$ versus $10-25 \mathrm{keV}$, and $3-10 \mathrm{keV}$ versus $20-60 \mathrm{keV}$, as is seen for GX 354-0 (see above). Again, most of the time there is an anti-correlation between the fluxes in $3-10 \mathrm{keV}$ and 20-60 keV energy bands.

GX 13+1. Previous INTEGRAL observations showed the 20-40 keV flux to be quickly varying from the IBIS/ISGRI detection limits (upper limit typically $5 \mathrm{mCrab}$ ) up to $\simeq 35 \mathrm{mCrab}$ on $\mathrm{a} \simeq 10$-day time scale (Paizis et al. 2006). In our program the source varies between the IBIS/ISGRI detection limits and $40 \mathrm{mCrab}(20-60 \mathrm{keV})$ on a revolution to revolution basis (Fig. 7), consistent with that seen previously.

$4 U 1722-30$ (in Terzan 2). The source $4 U 1722-30$ is persistently visible between $\simeq 10-25 \mathrm{mCrab}(20-60 \mathrm{keV})$. GRANAT/SIGMA saw the source varying between $\simeq 10-40 \mathrm{mCrab}(35-75 \mathrm{keV})$ on a $\sim 200$-day time scale (Goldwurm et al. 1995; see also Barret et al. 1991). During the middle of our second season it showed a drop to near IBIS/ISGRI detection levels for a time period of $\simeq 2$ weeks (September/October 2005; Fig. 7). Strong type I X-ray bursts are seen in all seasons, for a total of 5 .

$G X 3+1$. During the February 2003 to May 2004 period the $R X T E / A S M \quad 2-12 \mathrm{keV}$ intensity decreased more or less monotonically from about $400 \mathrm{mCrab}$ to about $250 \mathrm{mCrab}$. The $\simeq 2$-month average hard X-ray flux $(22-40 \mathrm{keV})$ decreased in the same period from about $15 \mathrm{mCrab}$ to about $8 \mathrm{mCrab}$. Within these 2 months periods the flux varied only weakly on a $\simeq 10$-day time scale (Paizis et al. 2006). Since May 2004 up to 


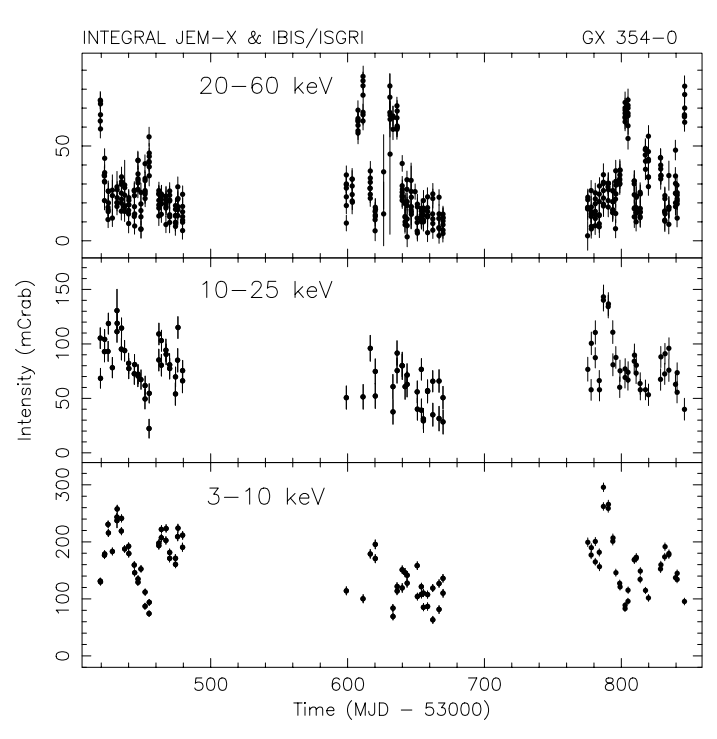

Fig. 8. INTEGRAL IBIS/ISGRI (20-60 keV, top) and JEM-X (3-10 keV, bottom; $10-25 \mathrm{keV}$, middle) light curves (data from single 1800 s pointings) of GX 354-0.

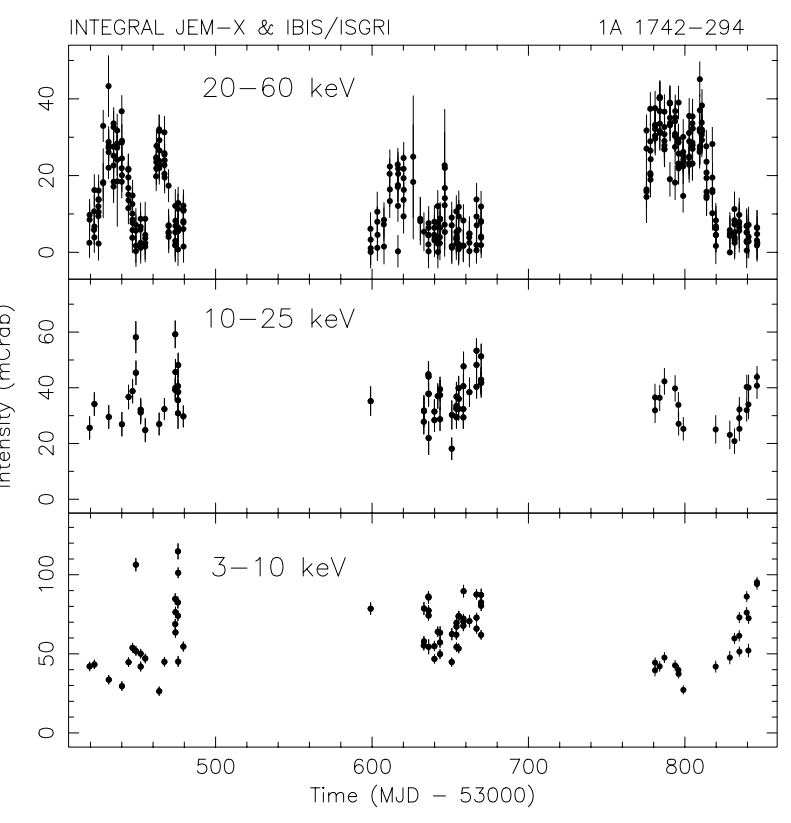

Fig. 9. Same as Fig. 8 but for 1A 1742-294.

August 2004 the source continued to decline to about $150 \mathrm{mCrab}$ in the $2-12 \mathrm{keV}$ band. Thereafter, it varied erratically on a roughly 100 day time scale between 100 and $250 \mathrm{mCrab}$ up to the end of 2005 . Over the course of 2006 the $2-12 \mathrm{keV}$ flux has been increasing steadily again; it was about $250 \mathrm{mCrab}$ at the end of our third season. The source is barely detectable in the $20-60 \mathrm{keV}$ band around $\simeq 10 \mathrm{mCrab}$ in our program (Fig. 7), consistent with the source behaviour in both soft and hard X-rays around May 2004 reported by Paizis et al. (2006). During the whole monitoring program we observed 10 type I X-ray bursts.

SLX 1735-269. We see SLX 1735-269 between 8-20 mCrab (20-60 keV; Fig. 7), i.e., just above the detection limits. This is typical of the source (Goldwurm et al. 1996), although INTEGRAL observations previous to our monitoring program show that occasionally the hard X-ray flux decreases below $\simeq 5 \mathrm{mCrab}$ (Molkov et al. 2005a). We saw no type I X-ray bursts during our observations with JEM-X.
GRS 1741.9-2853. GRS 1741.9-2853 is, similar to SAX J1747.0-2853, a faint X-ray transient source (e.g., Muno et al. 2003b). A hard X-ray outburst, with a peak of $\simeq 15 \mathrm{mCrab}$ and a duration of at least a couple of weeks $(20-60 \mathrm{keV})$ was seen near the end of the first visibility season (April 2005, MJD 53464-53479; see Fig. 7). We note that XMM-Newton and Chandra found the source to be still active in soft X-rays $(<10 \mathrm{keV})$, respectively, two and three months later (Wijnands et al. 2006). A previous detection at hard energies (40-100 keV) was reported by GRANAT/SIGMA in March/April 1990 (Churazov et al. 1993). No type I X-ray bursts were seen with JEM-X.

SLX 1744-299/300. SLX 1744-299/300 weakly varies. Fluxes up to $\simeq 15 \mathrm{mCrab}(20-60 \mathrm{keV})$ were reached during the second season (Fig. 7). A total of 9 type I X-ray bursts were seen in our data to come from them.

SLX 1737-282. SLX 1737-282 is a weak persistent X-ray source (Skinner et al. 1987; in 't Zand et al. 2002), and detected in the hard X-ray band $(3.4 \pm 0.2 \mathrm{mCrab}, 18-60 \mathrm{keV}$, Revnivtsev et al. 2004a; see also Bird et al. 2004, 2006). We see it varying between the detection limits up to about $12 \mathrm{mCrab}$ on a revolution time scale (20-60 keV; Fig. 7). In our JEM-X data we saw no type I X-ray bursts.

$X B$ 1832-330 (in NGC 6652). The globular cluster source XB 1832-330 lies far off-axis from the Galactic Center $\left(\simeq 11.5^{\circ}\right)$. We see it is a weak hard X-ray source with 20-60 keV fluxes between $\sim 10-20 \mathrm{mCrab}$ (Fig. 7). The source was seen at similar flux levels, without strong variability, during several INTEGRAL IBIS/ISGRI observations taken between March 2003 and September 2005 (Tarana et al. 2006b). We saw no type I X-ray bursts.

\subsubsection{Other low-mass $X$-ray binaries}

GX 5-1. Like GX 17+2 (Sect. 4.2.2), GX 5-1 is highly variable on various time scales (see Fig. 10). It is one of the brightest persistent sources in the canonical $2-10 \mathrm{keV}$ band (only Sco X-1 and Crab are brighter); it is also a Z source (Hasinger $\&$ van der Klis 1985). Thanks to the high angular resolution of IBIS/ISGRI we are able to discriminate clearly its hardenergy radiation from that of the nearby $\left(\simeq 40^{\prime}\right)$ strong hard X-ray source GRS 1758-258 (Sect. 4.2.1). On time scales of half an hour (one pointing) and longer also at harder energies (20-60 keV) the flux changes considerably, from the detection limit up to $\simeq 100 \mathrm{mCrab}$ (Fig. 10; see also Paizis et al. 2005, 2006 for previous INTEGRAL observations). Markert et al. (1979) do provide 15-40 keV long-term light curves for GX 5-1, but they are most likely contaminated by GRS $1758-258$.

GX 5-1 is the brightest source seen in the JEM-X fieldof-view of the Galactic bulge observations (see Fig. 4). It is so bright $(\simeq 700-1600 \mathrm{mCrab}$ and $\simeq 100-600 \mathrm{mCrab}$, in the $3-10 \mathrm{keV}$ and $10-25 \mathrm{keV}$ bands, respectively; Fig. 12) that it dominates the JEM-X detector count rates, which considerably influences the image reconstruction and therefore the quality of the observations. GX 5-1 shows a two-branch behaviour, both in the $3-10 \mathrm{keV}$ versus $10-25 \mathrm{keV}$ and $3-10 \mathrm{keV}$ versus $20-60 \mathrm{keV}$ bands (Fig. 13, left panel). In one branch there is a correlation in the intensities between the lowest X-ray band and the higher X-ray bands. In the other branch, which is connected to the former one at the highest intensities, there is (almost) no correlation: whereas the $3-10 \mathrm{keV}$ intensity varies, the $10-25 \mathrm{keV}$ and $20-60 \mathrm{keV}$ stay (almost) constant. This twobranch behaviour is related to the so-called " $\mathrm{Z}$ " branches seen in this source and other Z-sources. GX 5-1 is mostly seen in the 

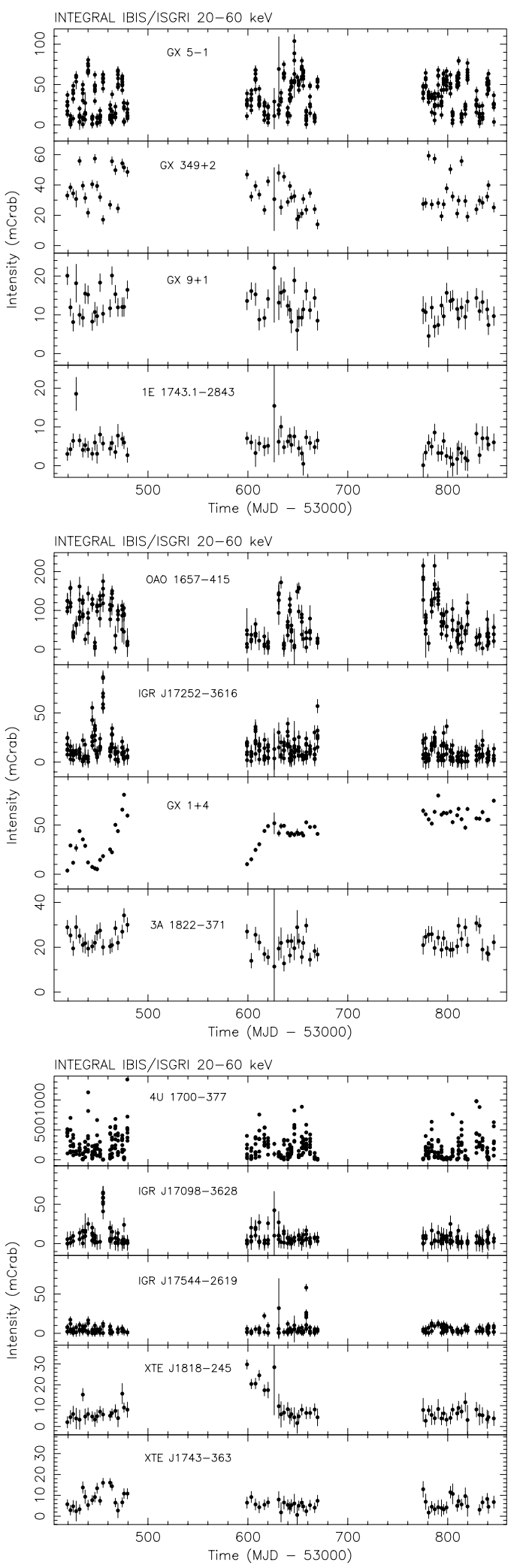

Fig. 10. Same as Fig. 7, but showing the following sources: top: (other low-mass X-ray binaries) GX 5-1, GX 349+2, GX 9+1, and 1E 1743.1-2843; middle: (X-ray pulsars) OAO 1657-415, IGRJ 17252-3616, GX 1+4, and 3A 1822-371; bottom: (miscellaneous sources) 4U 1700-377, IGR J17098-3628, IGR J17544-2619, XTE J1818-245, and XTE J1743-363.

so-called horizontal and normal branch (e.g., Kuulkers et al. 1994). This is also reflected in the hardness versus intensity

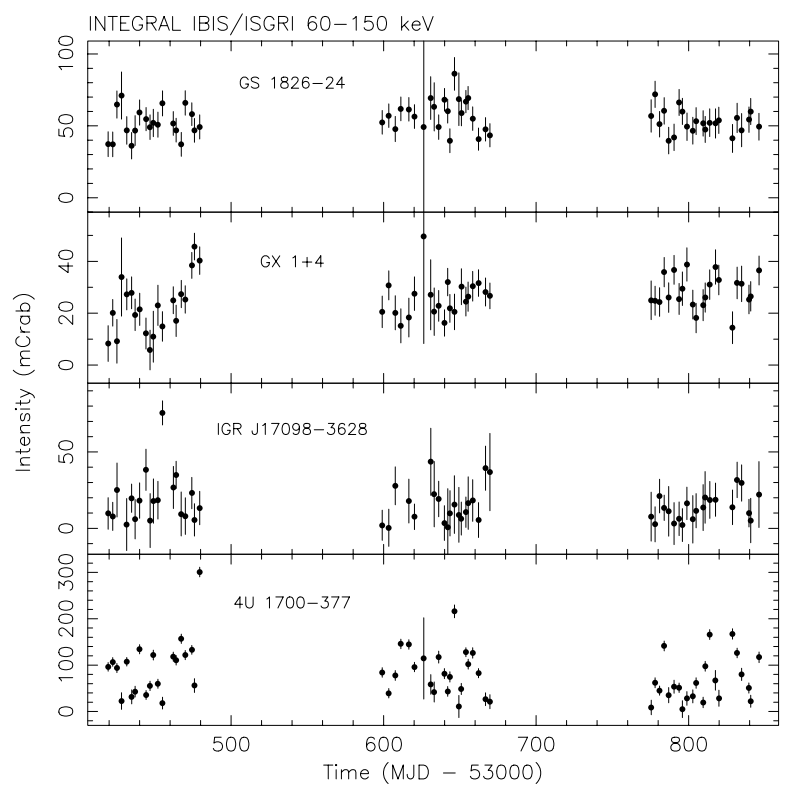

Fig. 11. INTEGRAL IBIS/ISGRI light curves $(60-150 \mathrm{keV})$ from the three seasons of the Galactic bulge monitoring program. Shown are the averages per hexagonal dither observation for the following sources: GS 1826-24, GX 1+4, IGR J17098-3628, and 4U 1700-377.

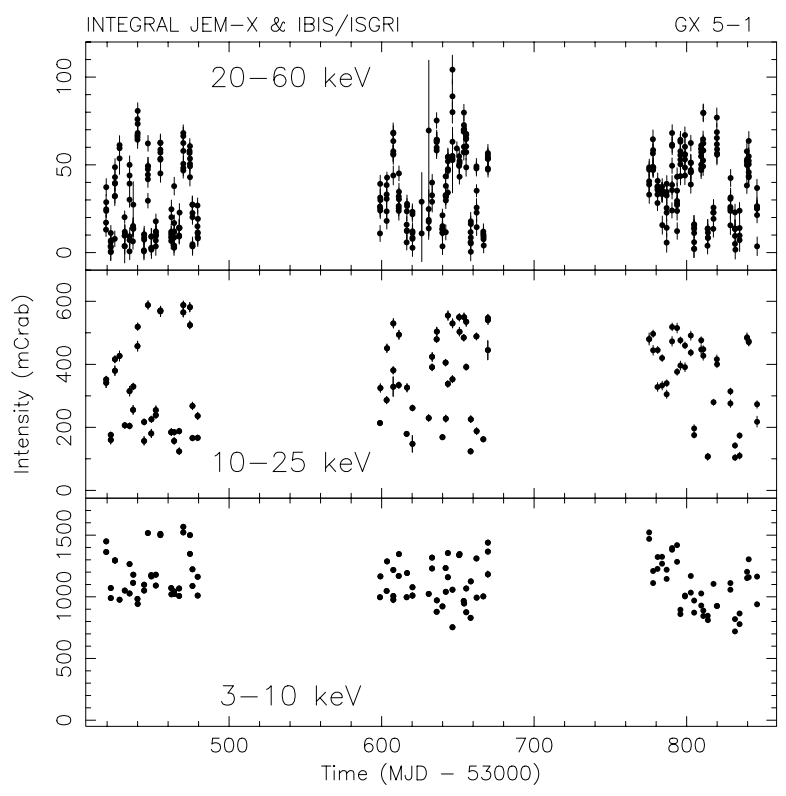

Fig. 12. Same as Fig. 8 but for GX 5-1.

(flux) diagram (HID; Fig. 13, middle panel). The horizontal branch runs from top left to middle right, the normal branch from middle right to bottom left. The HID is qualitatively similar to that drawn from other (all-sky) monitoring observations (Blom et al. 1993; van der Klis et al. 1991; Paizis et al. 2005). There is no evidence for such branch behaviour in the hardness versus the 20-60 keV flux (Fig. 13, right panel); in that case the hardness just increases linearly with increasing $20-60 \mathrm{keV}$ flux.

$G X 349+2$. Like GX 5-1 and GX 17+2, also GX 349+2 is a Z-source (Hasinger \& van der Klis 1989). It is highly variable between 15 and $60 \mathrm{mCrab}$ (20-60 keV; Fig. 10). Previous IBIS/ISGRI observations show similar flux variations on a $\simeq 10$-day time scale (22-40 keV; Paizis et al. 2006). 

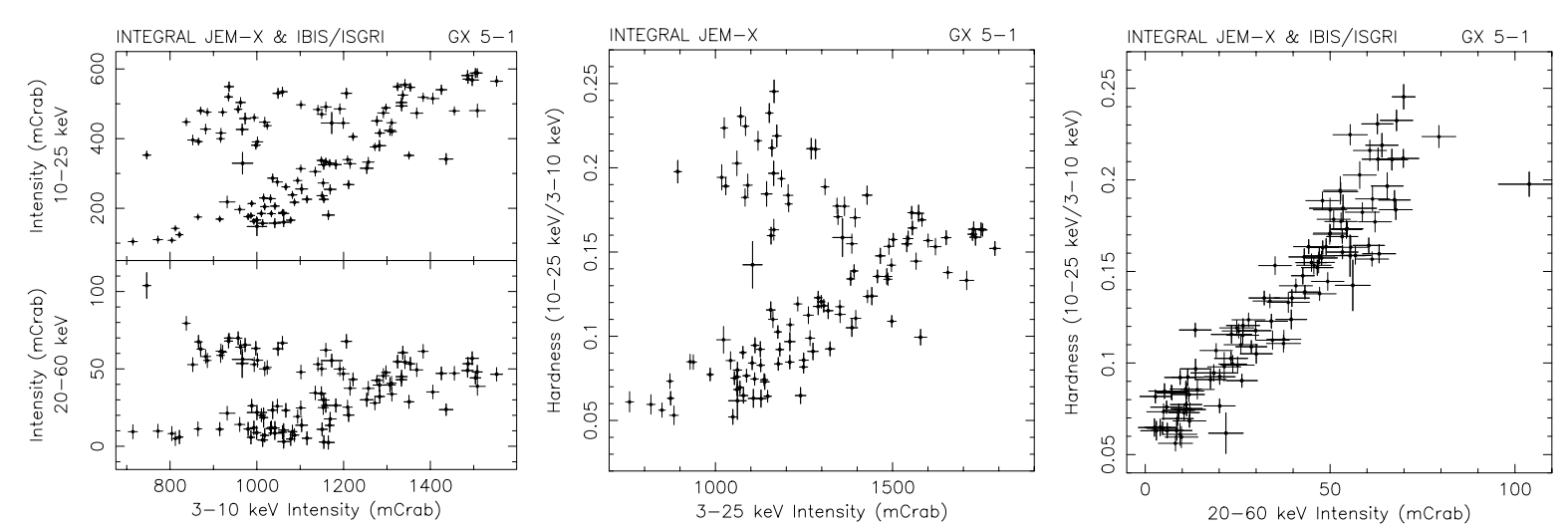

Fig. 13. Left: $10-25 \mathrm{keV}$ and $20-60 \mathrm{keV}$ intensities versus $3-10 \mathrm{keV}$ intensity for GX 5-1. Middle: Hardness (ratio of the count rates in the $10-25 \mathrm{keV}$ and 3-10 keV bands) versus intensity (3-25 keV) for GX 5-1. Right: Hardness versus intensity (20-60 keV) for GX 5-1.

$G X 9+1$. GX 9+1 varies from revolution to revolution, up to $\simeq 20 \mathrm{mCrab}(20-60 \mathrm{keV}$; Fig. 10$)$. On a $\simeq 10$-day time scale the source has been seen to weakly vary between $\simeq 10$ and $\simeq 20 \mathrm{mCrab}$ in the $22-40 \mathrm{keV}$ band, using previous INTEGRAL observations (Paizis et al. 2006).

IE 1743.1-2843. 1E 1743.1-2843 is a fairly persistent source in the Galactic Center region with a $20-40 \mathrm{keV}$ flux of $\simeq 5 \mathrm{mCrab}$; it only shows marginal variability over a few months time scale (Del Santo et al. 2006). Our monitoring is consistent with this (Fig. 10); 1E 1743.1-2843 is not significantly detected in the average of the first and third season, but it is during the second season (see Fig. 3, Table 3). The average flux over the second season is $4.4 \pm 0.2 \mathrm{mCrab}(20-60 \mathrm{keV})$, similar to that reported by Del Santo et al. (2006). We note that the high data point near the end of February 2005 (MJD 53428) is instrumental; the source was not significantly detected during that particular revolution (290).

\subsubsection{X-ray pulsars}

OAO 1657-415. The source is a high-mass X-ray binary with a pulse period of $38 \mathrm{~s}$ and an orbital period of 10.4 days (Chakrabarty et al. 1993). It is highly variable on monthly and longer time scales as seen by $C G R O / \mathrm{BATSE}$, reaching up to $\simeq 200 \mathrm{mCrab}(20-100 \mathrm{keV}$; Harmon et al. 2004). Like 4U 1700-377 (see Sect. 4.2.5), the hard X-ray flux modulates with the orbital period (e.g., Laycock et al. 2003). We see OAO $1657-415$ far off-axis from the Galactic Center $\left(\simeq 15.6^{\circ}\right)$; the $20-60 \mathrm{keV}$ and $60-150 \mathrm{keV}$ fluxes vary on a single pointing basis, and range between the IBIS/ISGRI detection limits and $\simeq 200 \mathrm{mCrab}$ (Fig. 10) and $\simeq 100 \mathrm{mCrab}$, respectively. During the first few weeks of the second season the source was not very active, compared to the rest of the observations.

IGR J17252-3616. IGR J17252-3616 was discovered in February 2004 (Walter et al. 2004). It has been found to show a pulse period of $414 \mathrm{~s}$ and an orbital period of 9.72 days (Zurita Heras et al. 2006). INTEGRAL/IBIS monitoring of IGR J17252-3616 indicates a mean $20-60 \mathrm{keV}$ flux of $\simeq 6.4 \mathrm{mCrab}$; the source was not detected in the $60-150 \mathrm{keV}$ band with $3 \sigma$ upper limits of typically $\simeq 7 \mathrm{mCrab}$. Every now and then IGR J17252-3616 flares on $<1$ day time scales up to about $70 \mathrm{mCrab}$ (Zurita Heras et al. 2006). This is consistent with the fact that most of the time we do not see the source, and our detection of a couple of flares, one of which occurred on March 26, 2005 (MJD 53820), with fluxes up to $90 \mathrm{mCrab}$ (20-60 keV; Fig. 10).
$G X 1+4$. GX $1+4$ is a symbiotic binary composed of a giant star and a neutron star (Chakrabarty \& Roche 1997; Belczy ński et al. 2000), with an orbital period of $\simeq 304$ days (Pereira et al. 1999) and a spin period of about 2 min (e.g., Lewin et al. 1971). INTEGRAL observations between March 2003 and October 2004 showed the source evolving from a weak intensity state in the beginning at about $20 \mathrm{mCrab}$ to a brighter intensity state at the end at about $120 \mathrm{mCrab}(20-40 \mathrm{keV})$. The source showed strong variability by a factor of $\simeq 10$ on a few $1000 \mathrm{~s}$ time scale on some occassions (Ferrigno et al. 2007). We detect the source at least up to $150 \mathrm{keV}$ (see also Ferrigno et al. 2007); it clearly varies on weekly and longer time scales from $\simeq 5-85 \mathrm{mCrab}$ and $\simeq 5-50 \mathrm{mCrab}(20-60 \mathrm{keV}$ and $60-150 \mathrm{keV}$, respectively; see Figs. 10 and 11). Note that the correlated variability between the $20-60 \mathrm{keV}$ and $60-150 \mathrm{keV}$ bands, present during the first season, is absent during the first part of the second season. GRANAT/SIGMA observations already showed similar variability at energies $\gtrsim 40 \mathrm{keV}$ at monthly time scales (Cordier et al. 1993; Mandrou et al. 1994; David et al. 1998) and half-a-year time scales (Goldwurm et al. 1995). $C G R O /$ BATSE shows clearly the $20-100 \mathrm{keV}$ variability on time scales longer than a month with fluxes between below the detection limit to up to $\simeq 200 \mathrm{mCrab}$ (Harmon et al. 2004). During the $M I T / O S O-7$ observations the $15-40 \mathrm{keV}$ flux varied mostly within about 200 and $300 \mathrm{mCrab}$ on months time scales, with one possible flaring period up to about $600 \mathrm{mCrab}$ (Markert et al. 1979).

3 A 1822-371. 3A $1822-371$ is a $5.57 \mathrm{~h}$ dipping and eclipsing accretion-disk corona source (e.g., White et al. 1981), with a pulse period of $0.59 \mathrm{~s}$ (Jonker \& van der Klis 2001). It is a persistent source in the $20-60 \mathrm{keV}$ band, and we see it varying on time scales of typically a revolution to a couple of revolutions between $\simeq 12$ and $35 \mathrm{mCrab}(20-60 \mathrm{keV}$; Fig. 10). Previous INTEGRAL/IBIS and BeppoSAX/PDS observations showed that the $15-40 \mathrm{keV}$ flux is clearly modulated with the orbital period (Williams et al. 2004).

\subsubsection{Miscellaneous sources}

4U 1700-377. The high-mass X-ray binary 4U 1700-377 has an orbital period of 3.41 days (Jones et al. 1973). The nature of the compact object is still unknown (Gottwald et al. 1986; Clark et al. 2002). It shows the strongest flaring activity in our sample, with fluxes from near the IBIS/ISGRI detection limit to generally $\simeq 500 \mathrm{mCrab}(20-60 \mathrm{keV})$ within one observation. Flares with fluxes up to about $1500 \mathrm{mCrab}$ are seen in our 

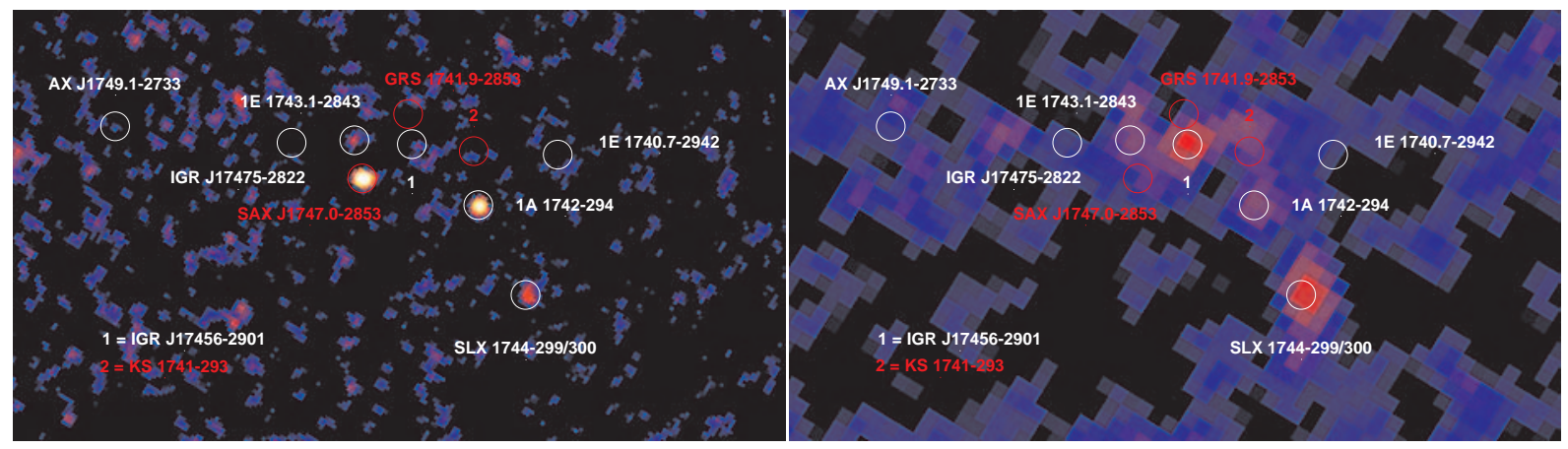

Fig. 14. INTEGRAL JEM-X (3-20 keV) (left) and IBIS/ISGRI (20-60 keV) (right) mosaic significance images of the Galactic Center region during revolutions 424-429 (April 3-21, 2006; MJD 53828-53846), for a total exposure of 69 ks. Note that the same scales are used as in Figs. 1-4. The annoted sources are those similar to Fig. 3. Clearly, most of the sources are "off" during that time. With JEM-X only SAX J1747.0-2853, 1A 1742-294 and SLX 1744-299/300 are significantly detected, whereas with IBIS/ISGRI only SLX 1744-299/300 and a source positionally coincident with IGR J17456-2901 are significantly detected. We refer to Sect. 4.3 for a more detailed discussion.

program (Fig. 10). In the $60-150 \mathrm{keV}$ band the source varies generally between the IBIS/ISGRI detection limits and $\simeq 200 \mathrm{mCrab}$ (Fig. 11), occasionally flaring reaching up to $\simeq 500 \mathrm{mCrab}$ in single pointings. This is similar to the hard $\mathrm{X}$ ray behaviour seen in previous observations by INTEGRAL, CGRO/BATSE, GRANAT/SIGMA, as well as older experiments; the lowest fluxes are reached during eclipse (Orr et al. 2004; Laycock et al. 2003; Kudryavtsev et al. 2001; Rubin et al. 1996; Laurent et al. 1992; Pietsch et al. 1980; Markert et al. 1979).

IGR J17098-3628. IGR J17098-3628 was discovered end of March 2005 (MJD 53453) by INTEGRAL with $18-45 \mathrm{keV}$ and $45-80 \mathrm{keV}$ fluxes of $\simeq 28$ and $\simeq 39 \mathrm{mCrab}$, respectively (Grebenev et al. 2005a). Near the peak the source spectrum changed significantly (Grebenev et al. 2005b). Our observations caught the source near the peak and we saw it fading away (Mowlavi et al. 2005). On March 26 (MJD 53455) the source showed considerable variability, up to $65 \mathrm{mCrab}(20-60 \mathrm{keV}$; Fig. 10); on that date a significant detection was also made in the $60-150 \mathrm{keV}$ band, with an average flux of $\simeq 75 \mathrm{mCrab}$ (Fig. 11; see also Mowlavi et al. 2005).

IGR J17544-2619. On 17 September, 2003 INTEGRAL discovered IGR J17544-2619 (Sunyaev et al. 2003b). It flared on time scales of hours with fluxes up to $\simeq 160 \mathrm{mCrab}$, $60 \mathrm{mCrab}$ and $\lesssim 15 \mathrm{mCrab}$ in the $18-25,25-50$ and $50-100 \mathrm{keV}$ IBIS/ISGRI bands (Sunyaev et al. 2003b; Grebenev et al. 2003; see also Walter et al. 2006). Earlier activity was seen by IBIS/ISGRI in April 2003; it was found flaring again near the end of February 2004 (Walter et al. 2006), as well as on March 8, 2004 (Grebenev et al. 2004a). On the latter date the source reached $17-45 \mathrm{keV}$ peak fluxes of $\simeq 160 \mathrm{mCrab}$ (Grebenev et al. 2004a). Walter et al. (2006) reported flux increases from the detection limits up to $\simeq 1000 \mathrm{mCrab}$ $(15-30 \mathrm{keV})$ within 5 min during the periods of activity in 2003 and 2004. Our monitoring shows that it flared again on October 15, 2005 (MJD 53658), up to $\simeq 60 \mathrm{mCrab}$ (20-60 keV; Fig. 10). Walter et al. (2006) suggested a period of $165 \pm 5$ days between activity (based on three flaring periods); however, our observations do not support this.

XTE J1818-245. On August 12, 2005 (MJD 53594) a new source, XTE J1818-245, was reported (Levine et al. 2005). This was just before the start of the second season, and the first observations showed the source to be bright, $\simeq 30 \mathrm{mCrab}(20-60 \mathrm{keV}$; Shaw et al. 2005). The transient faded more or less monitonically and went below the detection limits within a month (Fig. 10). During the first few monitoring observations of the same period the source was seen at $60-150 \mathrm{keV}$ as well, with fluxes between $\simeq 30-40 \mathrm{mCrab}$.

XTE J1743-363. This source was first detected in outburst by the RXTE/PCA in 1999 (Markwardt et al. 1999). It was marginally $(1.7 \pm 0.2 \mathrm{mCrab})$ detected by IBIS/ISGRI in September 2003 (Revnivtsev et al. 2004a), but it became active again in September 2004, with a mean flux of $\simeq 10$ mCrab (18-45 keV; Grebenev \& Sunyaev 2004a). We see clear activity up to $\simeq 18 \mathrm{mCrab}(20-60 \mathrm{keV})$ in the period March-April 2005 (MJD 53435-53479), during the first season (Fig. 10).

\subsection{April 2006: a quiet Galactic Center region}

It was already noted (Sect. 4.2.1) that 1E 1740.7-2942 was not detected during the third monitoring season. A close inspection of the $20-60 \mathrm{keV}$ light curve reveals that most of the other bright and/or transient X-ray sources in the Galactic Center region (1A 1742-294, SLX 1744-299/300, KS 1741-293, GRS 1741.9-2853, SAX J1747.0-2853) had faded during our monitoring observations performed on April 3-21, 2006. These observations clearly show the dynamic range in source activity present in the Galactic Center region.

In Fig. 14 we show the average mosaic image of the region in the $3-20 \mathrm{keV}$ and $20-60 \mathrm{keV}$ bands. Clearly, 1A 1742-294 and SAXJ1747.0-2853 were in a soft X-ray state during that time, whereas the transients KS 1741-293 and GRS 1741.9-2853 were off. A source in a hard X-ray state is seen ( $\$ 6 \mathrm{mCrab}, 3-20 \mathrm{keV} ; 9 \sigma, 4.6 \pm 0.5 \mathrm{mCrab}$, $20-60 \mathrm{keV})$, positionally coincident in our source sample with IGR J17456-2901. We derive a position of this source of $(\mathrm{J} 2000.0) \mathrm{RA}$, Dec $=266.410^{\circ},-29.029^{\circ}$, with a $90 \%$ confidence error of $2.9^{\prime}$. Within the quoted error of this Galactic Center source various (transient) X-ray binaries are known: A1742-289 (Eyles et al. 1975), AX J1745.6-2901 (Sakano et al. 2002), CXOGC J174538.0-290022, CXOGC J174541.0-290014， CXOGC J174535.5-290124 (Muno et al. 2003a), CXOGC J174540.0-290005 (Muno et al. 2005a), CXOGCJ174540.0-290031 (Muno et al. 2005b), SWIFT J174535.5-290135.6 (Kennea et al. 2006a) and SWIFT J17454.0-290005.3 (Kennea et al. 2006b,c). We have no (soft X-ray) information available of which one of these sources was active during our April 2006 observations, and thus can not securely identify the origin of the hard X-ray emission with one of them. On the other hand, the $20-60 \mathrm{keV}$ flux of our source is similar to that seen from IGR J17456-2901, which 


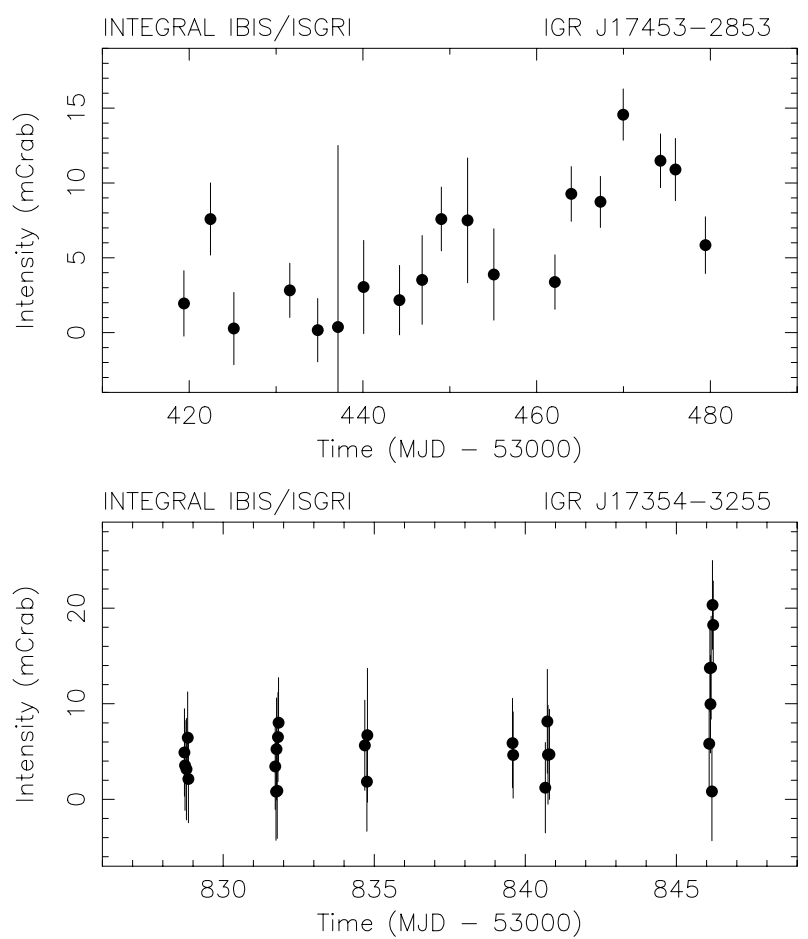

Fig. 15. INTEGRAL IBIS/ISGRI 20-60 keV light curves of IGR J17453-2853 (Top) and IGR J17354-3255 (Bottom) around and during the time of their detection.

has been constant over the two years before our monitoring observations (Bélanger et al. 2006). Note that none of the above mentioned X-ray binaries and X-ray transients fall within the error circle of IGR J17456-2901 (see Bélanger et al. 2006). We, therefore, tentatively identify our source with the steady source IGR J17456-2901, which we could detect significantly using a shorter exposure time, since no other closeby (bright) sources could contaminate the observations.

\subsection{New INTEGRAL sources}

Apart from the sources described in the previous sections, during the course of the third season we found several new X-ray sources (see Table 7). Also, a re-analysis of the whole Galactic bulge monitoring data set is in progress, and in the course of this exercise we also find new X-ray sources. Since these sources were not part (yet) of our catalog of sources, they are not included in the discussion on the images (Sect. 4.1) and long-term light curves (Sect. 4.2). Here we only shortly summarize their properties as described in various ATels, or report on first results from the re-analysis on the new INTEGRAL sources.

$I G R J 17453-2853$. In the observations performed on April 3, 2005 (MJD 53463) a new source is detected by IBIS/ISGRI at the $\simeq 6 \sigma$ level with a $20-60 \mathrm{keV}$ flux of $\simeq 7 \mathrm{mCrab}$. It was seen on average at the same level in the following observations, up to April 14, 2005 (MJD 53474; see Fig. 15). The best-fit position is given in Table 7 . We designate this source IGR J17453-2853. Despite being at a favourable offaxis angle from the Galactic Center $\left(\simeq 2^{\circ}\right)$, IGR J17453-2853 is not seen with JEM-X $(\$ 6 \mathrm{mCrab}$ and $\$ 7.5 \mathrm{mCrab}$, for $3-10 \mathrm{keV}$ and $10-25 \mathrm{keV}$, respectively), potentially indicating high intrinsic absorption or that it underwent a hard X-ray outburst. The source is rapidly variable, with the $20-60 \mathrm{keV}$ flux changing by a factor of $\simeq 2$ on a time scale of about half an hour. It reached a
Table 7. New INTEGRAL sources detected through our monitoring program. We give the source position (J2000.0) with its $90 \%$ confidence error, whether the source was detected by JEM-X (J) and/or IBIS/ISGRI (I) or not, and the reference in which the detection was first noted. References: [1] Kuulkers et al. (2006c); [2] this paper; [3] Turler et al. (2006); [4] Chenevez et al. (2006b).

\begin{tabular}{l|ccccc}
\hline \hline Source & \multicolumn{2}{|c}{$\begin{array}{c}\text { RA } \\
\text { (degrees) }\end{array}$} & err & J/I? & Reference \\
\hline IGR J17354-3255 & 263.854 & -32.922 & $4^{\prime}$ & no & \\
IGR J1745es & {$[1]$} \\
IGR J17453-2853 & 266.329 & -27.039 & $1^{\prime}$ & yes/no & {$[2]$} \\
IGR J17536-2339 & 266.328 & -28.891 & $2^{\prime}$ & no/yes & {$[2]$} \\
IGR J17541-2252 & 268.409 & -23.654 & $4^{\prime}$ & no $^{b} /$ yes & {$[3]$} \\
IGR J17456-2901b & 268.517 & -22.871 & $4^{\prime}$ & no $^{b} /$ yes & {$[3]$} \\
\hline
\end{tabular}

${ }^{a}$ JEM-X instrument was off. ${ }^{b}$ Not in the JEM-X field of view.

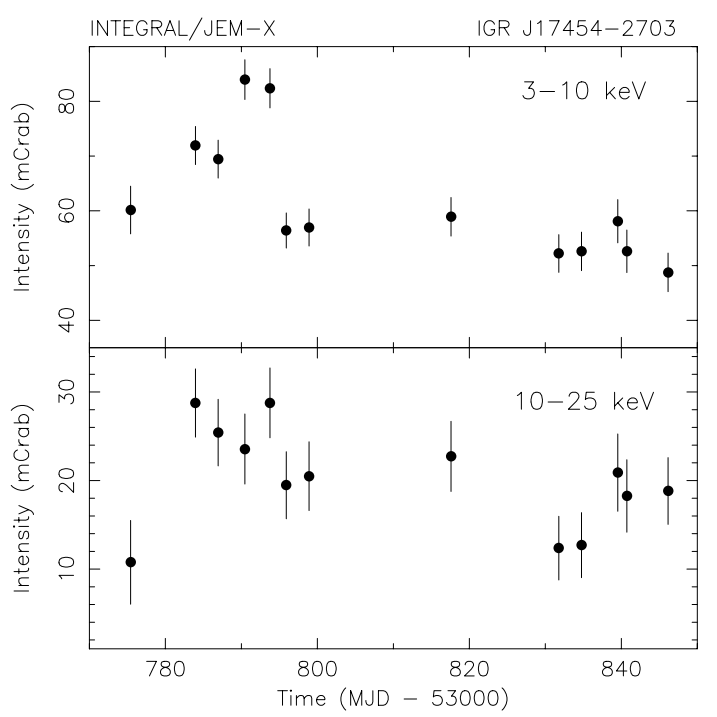

Fig. 16. INTEGRAL JEM-X 3-10 keV (Top) and 10-25 keV (Bottom) light curve of IGR J17454-2703 during the third visibility season. Data points are from single pointings whenever the source was within the JEM-X field of view.

peak flux $27 \pm 4$ mCrab on April 10, 2005 (MJD 53470; Fig. 15). A preliminary spectral analysis shows that the source spectrum can be described by a power-law with index of $\simeq 2.2$, indeed indicating a hard spectrum. We note that if the non-detection with JEM-X is due to absorption in the line of sight, $N_{\mathrm{H}}$ should be larger than about $10^{23}$ atoms $\mathrm{cm}^{-2}$.

IGR J17454-2703. Combining our monitoring observations performed between February 17 and March 4, 2006 (MJD 53783-53798), a new source is found with JEM-X. The position is given in Table 7 . We designate the source IGR J17454-2703. No previously known X-ray source is found within $5^{\prime}$ of this position in the Simbad database. The light curve of this new X-ray transient shows that it was at its maximum between February 24 and 27 (MJD 53790-53793; Fig. 16). In one of the single pointings on February 27 the source reached a peak flux of $\simeq 82 \mathrm{mCrab}(3-10 \mathrm{keV})$ and $\simeq 29 \mathrm{mCrab}(10-25 \mathrm{keV})$. We note that the 3-20 keV JEM-X spectrum can be modeled by an absorbed power law with an absorption column, $N_{\mathrm{H}}$ of $\simeq 1.2 \times$ $10^{23}$ atoms cm ${ }^{-2}$ and photon index of $\simeq 3.5$. We do not detect the source with IBIS/ISGRI, either by combining the observations between February 17 and March 4 ( $\$ 2 \mathrm{mCrab}, 20-60 \mathrm{keV}$ ), or in single hexagonal dither observations ( $\$ 4 \mathrm{mCrab}, 20-60 \mathrm{keV}$ ), consistent with the steep soft X-ray spectrum. 
IGR J17456-2901b. A new X-ray transient, SWIFT J174535.5-290135.6, was detected on February 25, 2006 (MJD 53791). It was not seen the previous day, and showed a power-law-like decay in flux the days after (Kennea et al. 2006a). We (Chenevez et al. 2006b) reported on a source coincident with this new Swift transient, which was seen about a week earlier with JEM-X and IBIS/ISGRI with fluxes of about $9 \mathrm{mCrab}(6-10 \mathrm{keV})$ and about $6 \mathrm{mCrab}(20-60 \mathrm{keV})$, and, therefore, associated with SWIFT J174535.5-290135.6. However, the Swift transient was not seen on February 24, and more X-ray (transient) sources are known (see Sect. 4.3) within the JEM-X error circle (Table 7). Most of our detected hard X-ray emission may come from IGR J17456-2901, but the transient soft X-ray emission does not (see Sect. 4.3; Bélanger et al. 2006). We conclude that at least our detected soft X-ray emission is due to a transient source, which we designate IGR J17456-2901b, but we can not attribute with confidence this transient emission to either SWIFT J174535.5-290135.6 or any other known source within the error circle.

IGR J17536-2339, IGR J17541-2252. In the beginning of April 2006 (MJD 53828) two new faint closeby $\left(\simeq 0.8^{\circ}\right)$ sources were detected by IBIS/ISGRI (see Table 7 for the position): IGR J17536-2339 and IGR J17541-2252. They had fluxes of $\simeq 11$ and $\simeq 10 \mathrm{mCrab}$, respectively, in the $20-60 \mathrm{keV}$ band. Within the positional errors IGR J17536-2339 is coincident with the nearby X-ray burster SAX J1753.5-2349 (Turler et al. 2006).

IGR J17354-3255. Another new source (see Table 7 for the position), IGRJ17354-3255, showed up in the last single pointings of our AO-3 program, i.e., those performed on April 21, 2006 (MJD 53846; Kuulkers et al. 2006c). It peaked to a $20-60 \mathrm{keV}$ flux of $\simeq 20 \mathrm{mCrab}$ (Fig. 15).

\subsection{OMC results}

Many of the sources we study do not have optical/IR counterparts or their optical counterparts are fainter than the OMC detection limit (mainly due to interstellar absorption). Since the Galactic bulge region is crowded, when observing a high-energy source which has a relatively large positional error circle, one may observe various possible counterparts, or the right counterpart may not even be visible. This is the case for most of our targets. However, whenever a source flares up in brightness, with concurrent brightening in hard X-rays $/ \gamma$-rays, we may be able to detect them with our analysis method.

One of the few examples, however, of a relatively clear field with an optical/IR counterpart is that of IGR J17544-2619. The OMC light curve of the counterpart (2MASS J17542527-2619526; Rodriguez 2003; Pellizza et al. 2006) is given in Fig. 17. The source does not vary much on a daily time scale, and is consistent with being constant near $V \simeq 12.9 \mathrm{mag}$, close to that seen previously (Pellizza et al. 2006). As shown by the simultaneous $20-60 \mathrm{keV}$ IBIS/ISGRI light curve in the same plot, the hard X-ray source was not active at that time.

\section{Summary and conclusions}

In this paper we have shown the results of the first one and a half year of monitoring of sources in and around the Galactic bulge region at soft $(3-25 \mathrm{keV})$ and hard $(20-150 \mathrm{keV}) \mathrm{X}$-ray energies, with a focus on the short, medium and long-term

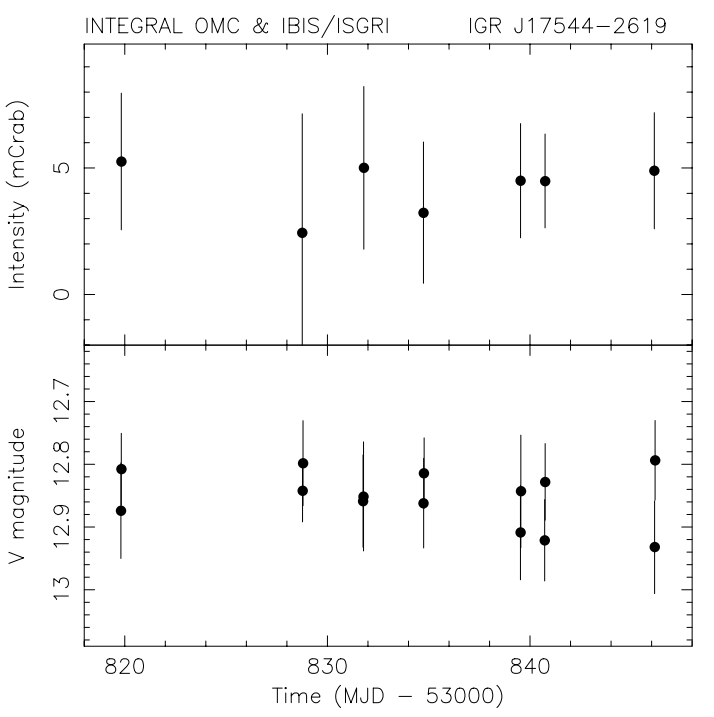

Fig. 17. Top: IBIS/ISGRI 20-60 keV light curve; data points are averages per hexagonal dither observation. Bottom: OMC light curve of the optical counterpart of IGR J17544-2619, i.e., 2MASS J17542527-2619526 (= IOMC 6849000050).

source variability within this period. For the first time we have performed a high-sensitivity $>20 \mathrm{keV}$ study of variability at hourly to weekly to monthly time scales, especially for the sources in the Galactic Center region, which has not been possible before due to source confusion of previous hard X-ray instruments. Our program has been succesfull in monitoring the hard X-ray variability of bright persistent sources, as well as characterising the (long-term) properties of old and new Xray transients. Moreover, due to the frequent monitoring we are able to detect fast X-ray transients, i.e., those which are typically active for less than a day. We also found six new INTEGRAL sources: IGR J17354-3255, IGR J17453-2853, IGR J17454-2703, IGR J17456-2901b, IGR J17536-2339, and IGR J17541-2252.

The fact that INTEGRAL has various instruments aboard which are sensitive in the soft and hard X-ray region, allows us to simultaneously monitor these energy ranges for (anti-) correlations, as well as fast phenomena such as X-ray bursts. Moreover, due to the wide field of view of the instruments we are able to monitor a large sample of X-ray sources all at the same time. It thus allows a homogeneous analysis of all the sources, similar to what was possible with the BeppoSAX/WFC at soft X-rays (see, e.g., in 't Zand 2001), and, to a lesser extent, with GRANAT/SIGMA at hard X-rays (see, e.g., Mandrou et al. 1994).

In the Galactic bulge and its surroundings we see about 15 persistent neutron-star low-mass X-ray binaries, 10 of which are X-ray bursters, active over the whole monitoring period at energies 20-60 keV. Per season we detect 22/23 sources above a detection significance of 7 . Of the black-hole (candidate) binaries in our sample we see the two persistent sources for most of the time, 1E 1740.7-2942 and GRS 1758-258. The former one was seen to turn off for a period of at least about three months. So far, we have seen one bright (exceeding $100 \mathrm{mCrab}, 20-60 \mathrm{keV}$ ) black-hole (candidate) X-ray transient (such as GRO J1655-40) in outburst per season, for a period of months. We see on average about three transient X-ray bursters active per season for periods ranging between weeks (e.g., GRS 1741.9-2853) to months (e.g., XTEJ1739-285) with peak fluxes of about 
$25 \mathrm{mCrab}(20-60 \mathrm{keV})$. The average number of fast X-ray transients (i.e., visible for only a couple of hours up to a day) per season is about one; they peak to about $100 \mathrm{mCrab}(20-60 \mathrm{keV}$; e.g., IGR J17544-2619). Most of the sources we detect on a hours to daily time scales are (transient) low-mass or high-mass $\mathrm{X}$-ray binaries. Those detected only using averages per season, i.e., the weaker sources with fluxes typically around $5 \mathrm{mCrab}$ (20-60 keV), also include cataclysmic variables and AGN.

Whereas, for example, GX 3+1 (X-ray burster) does not vary significantly, sources like GX $1+4$ (symbiotic neutronstar binary) and 1A 1742-294 (X-ray burster) vary on monthly times scales, while GRS 1758-258 (black-hole candidate) and GS 1826-24 (X-ray burster) generally vary smoothly on even longer time scales. As noted above, some sources clearly show transient behaviour, i.e., they show outbursts with durations exceeding months (e.g., H1743-322, a black-hole candidate), weeks (e.g., MXB 1730-335, an X-ray burster) or flaring on time scales of hours to days (e.g., IGR J17252-3616, an X-ray pulsar). For the X-ray transient XTEJ1739-285 we secured its identification to host a neutron star through our monitoring program (Brandt et al. 2005). Some sources vary on all time scales accessible through our program, as displayed by, e.g., the highmass X-ray binary 4U 1700-377. Hard X-ray flares/outbursts in X-ray burst sources (e.g., GX 354-0, H1820-30) are accompanied by soft X-ray drops, i.e., the spectra pivot somewhere between 10-20 keV when the sources become harder (see also Tarana et al. 2006a). The reverse is also seen (e.g., 4U 1722-30). GX 5-1, a bright non-bursting low-mass X-ray binary, on the other hand, shows no such anti-correlation. The soft X-ray emission is either correlated with the hard X-ray emission, or not, depending on the position in the " $Z$ "-diagram.

The temporal behaviour of hard X-ray emission from X-ray bursters has led to a division into three classes (see, e.g., Tavani \& Barret 1997): 1) X-ray bursters with persistent (though variable on a monthly time scale) hard X-ray emission (such as GS 1826-24), 2) X-ray bursters showing episodic (for 10-20 days) hard X-ray emission (such as GX 354-0), and 3) X-ray bursters which are transient (such as AqlX-1). The behaviour we describe above confirms this division, except that some of the persistent X-ray bursters may show temporary (for $\sim 10-20$ days) drops in their hard X-ray emission (e.g., $4 \mathrm{U} 1722-30$ ). The sparse examples of long-term simultaneous soft and hard X-ray observations of X-ray bursters (in the preINTEGRAL literature) indicate that hard X-rays are generally anti-correlated with soft X-rays (e.g., 4U 0614+091, Ford et al. 1996). However, we show that sometimes no (anti-)correlation is seen between soft and hard X-ray emission in X-ray bursters, i.e., the soft and hard X-ray can both be low.

At higher energies, i.e., $60-150 \mathrm{keV}$, we detect about $8 / 9$ sources per season above a detection significance of 7 , and about a dozen in the data covering the whole monitoring period. These are either transient or persistent black-hole candidates, Xray bursters or high-mass X-ray binaries. This is in accordance with the hard X-ray (>100 keV) INTEGRAL/IBIS survey (which includes the Galactic bulge region) by Bazzano et al. (2006), who concluded that the $100-150 \mathrm{keV}$ band is dominated by low and high-mass X-ray binaries.

The next step will be, apart from the continuation of the Galactic bulge monitoring in the future, the investigation of (hard X-ray) variability at smaller time scales (milliseconds to minutes) and the relation to long-term variability, such as timeresolved pulse timing and detailed X-ray burst analysis. We also plan to study the long-term energy spectral behaviour of our sources, as well as a comparison with other ongoing monitoring programs (e.g., with the RXTE/ASM and PCA).

In this introductory paper we have shown that most of the hard X-ray sources in the field of view of the INTEGRAL instruments included in our program clearly vary on time scales of a few hours to days to months. It is therefore no surprise that the Galactic bulge is a region to stay tuned to.

Acknowledgements. Based on observations with INTEGRAL, an ESA project with instruments and science data centre funded by ESA member states (especially the PI countries: Denmark, France, Germany, Italy, Switzerland, Spain), Czech Republic and Poland, and with the participation of Russia and the USA. This research has made use of the SIMBAD database, operated at CDS, Strasbourg, France. E.K. thanks Angela Bazzano and Guillaume Bélanger for comments on an earlier version of the paper.

Note added in proof. After the submission of our paper, we became aware that four sources in our sample which we label as unidentified in Table 1 can be classified in the following way: SAX J1818.6-1703: HMXB (Negueruela, I., \& Smith, D. M. 2006, ATel \#831; in 't Zand, J., Jonker, P., Mendez, M., \& Markwardt, M. 2006, ATel \#915); 1RXS J175113.3-201214 and IGR J17488-3253: AGN, Sy1; IGR J17200-3116: HMXB (Masetti, N., Morelli, L., Palazzi, E., et al. 2006, A\&A, 459, 21). Furthermore, we have recently found that our newly discovered source with IBIS/ISGRI, IGR J17453-42853, is most probably associated with GRS 1741.9-2853, based on the nearcontemporaneous observations by the position sensitive X-ray instrument Swift/XRT, a detailed analysis of recent JEM-X data, as well as their similar outburst characteristics (see Kuulkers, E., Shaw, S, Chevenez, J., et al. 2007, ATel \#1008).

\section{References}

Barthelmy, S. D. 2000, in Proc. SPIE, 4140, p. 50

Barret, D., Mereghetti, S., Roques, J. P., et al. 1991, ApJ, 379, L21

Barret, D., Grindlay, J. E., Bloser, P. F., et al. 1996, A\&AS, 120, 121 Bazzano, A., La Padula, C., Ubertini, P., \& Sood, R. K. 1992, ApJ, 385, L17

Bazzano, A., Bird, A. J., Cocchi, M., et al. 2004, in Proc. 5th INTEGRAL Science Workshop, The INTEGRAL Universe, ed. V. Schönfelder, G. Lichti, \& C. Winkler, ESA SP-552, 309

Bazzano, A., Stephen, J. B., Fiocchi, M., et al. 2006, ApJ, 649, L9 Bélanger, G., Goldwurm, A., Renaud, M., et al. 2006, ApJ, 636, 275 Belczyński, K., Mikołajewska, J., Munari, U., Ivison, R. J., \& Friedjung, M. 2000, A\&AS 146, 407

Bhattacharyya, S., Strohmayer, S. E., Swank, J. H., \& Markwardt, S. B. 2006, ApJ, 639, L31

Bird, A. J., Barlow, E. J., Bassani, L., et al. 2004, ApJ, 607, L33

Bird, A. J., Barlow, E. J., Bassani, L., et al. 2006, ApJ, 636, 765 Blom, J. J., in 't Zand, J. J. M., Heise, J., et al. 1993, A\&A, 277, 77 Bloser, P. F., Barret, D., \& Grindlay, J. E. 1996, A\&AS, 120, 275 Bodghee, A., Mowlavi, N., Kuulkers, E., et al. 2005, ATel \#592 Bouchet, L., Roques, J. P., Mandrou, P., et al. 2005, ApJ, 635, 1103 Brandt, S., Budtz-Jørgensen, C., \& Chenevez, J. 2006, ATel \#778 Brandt, S., Kuulkers, E., Bazzano, A., et al. 2005, ATel \#622 Brocksopp, C., McGowan, K. E., Krimm, H., et al. 2006, MNRAS, 365, 1203 Buxton, M., Bailyn, C., \& Maitra, D. 2005, ATel \#418

Capitanio, F., Ubertini, P., Bazzano, A., et al. 2005, ApJ, 622, 503 Chakrabarty, D., \& Roche, P. 1997, ApJ, 489, 254

Chakrabarty, D., Grunsfeld, J., Prince, T. A., et al. 1993, ApJ, 403, L33

Chelovekov, I. V., Grebenev, S. A., \& Sunyaev, R. A. 2006, AstL, 32, 456 Chenevez, J., Shaw, S. E., Kuulkers, E., et al. 2006a, ATel \#734

Chenevez, J., Sanchez-Fernandez, C., Kuulkers, E., et al. 2006b, ATel \#756

Chernyakova, M. 2005, IBIS Analysis User Manual, OSA 5.1

Chou, Y., \& Grindlay, J. E. 2001, ApJ, 563, 934

Churazov, E., Gilfanov, M., Sunyaev, R., et al. 1993, A\&AS, 97, 173 Churazov, E., Gilfanov, M., Sunyaev, R., et al. 1994, ApJS, 92, 381 Churazov, E., Gilfanov, M., Sunyaev, R., et al. 1995, ApJ, 443, 341 Claret, A., Goldwurm, A., Cordier, B., et al. 1994, ApJ, 423, 436 Clark, J. S., Goodwin, S. P., Crowther, P. A., et al. 2002, A\&A, 392, 909 Cocchi, M., Bazzano, A., Natalucci, L., et al. 1999, A\&A, 346, L45 Cordier, B., Goldwurm, A., Leray, J. P., et al. 1993, A\&AS, 97, 177 
Cook, W. R., Grunsfield, J. M., Heindl, W. A., et al. 1991, ApJ, 372, L75

Courvoisier, T. J.-L., Walter, R., Beckmann, V., et al. 2003, A\&A, 411, L53

David, P., Laurent, P., Denis, M., et al. 1998, A\&A, 332, 165

De Cesare, G., Bazzano, A., Stratta, G., et al. 2006, in The X-ray Universe 2005. ESA SP-604, 259

Del Santo, M., Bazzano, A., Zdziarski, A. A., et al. 2005, A\&A, 433, 613

Del Santo, M., Sidoli, L., Bazzano, A., et al. 2006, in The X-ray Universe 2005, ESA SP-604, 283

Deluit, S., Bazzano, A., Mowlavi, N., et al. 2004, ATel \#256

Ebisawa, K., Bourban, G., Bodaghee, A., Mowlavi, N., \& Courvoisier, T. J.-L. 2003, A\&A, 411, L59

Eyles, C. J., Skinner, G. K., Willmore, A. P., \& Rosenberg, F. D. 1975, Nature, 257, 291

Falanga, M., Farinelli, R., Goldoni, P., et al. 2004, A\&A, 426, 979

Falanga, M., Götz, D., Goldoni, P., et al. 2006, A\&A, 458, 21

Ferrigno, C., Segreto, A., Santangelo, A., et al. 2007, A\&A, 462, 995

Ford, E., Kaaret, P., Tavani, M., et al. 1996, ApJ, 469, L37

Forman, W., Jones, C., \& Tananbaum, H. 1976, ApJ, 207, L25

Gilfanov, M., Churazov, E., Sunyaev, R., et al. 1993, ApJ, 418, 844

Gottwald, M., White, N. E., \& Stella, L. 1986, MNRAS, 222, 21P

Goldoni, P., Kuulkers, E., Rodriguez, J., et al. 2006, ATel \#742

Goldwurm, A., Denis, M., Paul, J., et al. 1995, Adv. Space Res., 15, 41

Goldwurm, A., Vargas, M., Paul, J., et al. 1996, A\&A, 310, 857

Goldwurm, A., David, P., Foschini, L., et al. 2003, A\&A, 411, L223

Grebenev, S. A., \& Sunyaev, R. A. 2004a, ATel \#332

Grebenev, S. A., \& Sunyaev, R. A. 2004b, ATel \#342

Grebenev, S. A., Lutovinov, A. A., \& Sunyaev, R. A. 2003, ATel \#192

Grebenev, S. A., Rodriguez, J., Westergaard, N. J., Sunyaev, R. A., \& Oosterbroek, T. 2004a, ATel \#252

Grebenev, S. A., Revnivtsev, M. G., \& Sunyaev, R. A. 2004b, ATel \#257

Grebenev, S. A., Molkov, S. V., \& Sunyaev, R. A. 2005a, ATel \#444

Grebenev, S. A., Molkov, S. V., Revnivtsev, M. G., \& Sunyaev, R. A. 2005b, ATel \#447

Grebenev, S. A., Molkov, S. V., \& Sunyaev, R. A. 2005c, ATel \#616

Harmon, B. A., Wilson, C. A., Fishman, G. J., et al. 2004, ApJS, 154, 585

Hasinger, G., \& van der Klis, M. 1989, A\&A, 225, 79

Homan, J. 2005, ATel \#440

in 't Zand, J. 2001, in Exploring the gamma-ray universe, ed. A. Gimenez, V. Reglero, \& C. Winkler, ESA Publications Division, Noordwijk, ESA SP-459, 463

in 't Zand, J. J. M. 2005, A\&A, 441, L1

in 't Zand, J., Heise, J., Bazzano, A., et al. 1997, IAU Circ. 6618

in 't Zand, J., Verbunt, F., Kuulkers, E., et al. 2002, A\&A, 389, L43

in 't Zand, J. J. M., Hulleman, F., Markwardt, C. B., et al. 2003, A\&A, 406, 233

in 't Zand, J., Verbunt, F., Heise, J., et al. 2004, in BeppoSAX 2003, The Restless

High-Energy Universe, ed. E. P. J. van den Heuvel, J. J. M. in 't Zand, \&

R. A. M. J. Wijers, Nucl. Phys. B (Proc. Suppl.), 132, 486

Joinet, A., Jourdain, E., Malzac, J., et al. 2005, ApJ, 629, 1008

Jones, C., Forman, W., Tananbaum, H., et al. 1973, ApJ, 181, L43

Jonker, P. G., \& van der Klis, M. 2001, ApJ, 553, L43

Kalemci, E., Tomsick, J. A., Rothschild, R. E., et al. 2006, ApJ, 639, 340

Kennea, J. A., Burrows, D. N., Campana, S., et al. 2006a, ATel \#753

Kennea, J. A., Wijnands, R., Burrows, D. N., Nousek, J., \& Gehrels, N. 2006b, ATel \#920

Kennea, J. A., Wijnands, R., Burrows, D. N., Nousek, J., \& Gehrels, N. 2006c, ATel \#921

Knight, F. K., Johnson III, W. N., Kurfess, J. D., \& Strickman, M. S. 1985, ApJ, 290, 557

Kretschmar, P., Mereghetti, S., Hermsen, W., et al. 2004, ATel \#345

Kretschmar, P., Shaw, S. E., Kuulkers, E., et al. 2005, ATel \#593

Krimm, H., Barbier, L., Barthelmy, S. D., et al. 2006, ATel \#904

Kudryavtsev, M. I., Svertilov, S. I., \& Bogomolov, V. V. 2001, AstL, 27, 648

Kuulkers, E., van der Klis, M., Oosterbroek, T., et al. 1994, A\&A, 289, 795

Kuulkers, E., Shaw, S., Paizis, A., et al. 2005a, ATel \#438

Kuulkers, E., Kretschmar, P., Brandt, S., et al. 2005b, ATel \#642

Kuulkers, E., Goldoni, P., Shaw, S. E., et al. 2006a, ATel \#738

Kuulkers, E., Shaw, S., Brandt, S., et al. 2006b, in The Transient Milky Way: a perspective for MIRAX, ed. F. D’Amico, J. Braga, \& R. Rothschild, AIP Conf. Proc., 840, 30 [arXiv: astro-ph/0603130]

Kuulkers, E., Shaw, S., Paizis, A., et al. 2006c, ATel \#874

Kuznetsov, S. I., Gilfanov, M. R., Churazov, E. M., et al. 1999, AstL, 25, 351

Labanti, C., Di Cocco, G., Ferro, G., et al. 2003, A\&A, 411, L149

Laurent, P., Goldwurm, A., Lebrun, F., et al. 1992, A\&A, 260, 237

Laycock, S., Coe, M. J., Wilson, C. A., Harmon, B. A., \& Finger, M. 2003, MNRAS, 338, 211

Lebrun, F., Leray, J. P., Lavocat, P., et al. 2003, A\&A, 411, L141

Levine, A. M., Swank, J. H., Lin, D., \& Remillard, R. A. 2005, ATel \#578

Lewin, W. H. G., Ricker, G. R., \& McClintock, J. E. 1971, ApJ, 169, L17
Lowes, P., in 't Zand, J. J. M., Heise, J., et al. 2002, IAUC 7867

Lutovinov, A., Revnivtsev, M., Molkov, S., \& Sunyaev, R. 2005, A\&A, 430, 997

Lutovinov, A., Cadolle Bel, M., Belanger, G., et al. 2004, ATel \#328

Lund, N., Budtz-Jørgensen, Westergaard, N. J., et al. 2003, A\&A, 411, L231

Mandrou, P., Roques, J. P., Bouchet, L., et al. 1994, ApJS, 92, 343

Markert, T. H., Winkler, P. F., Laird, F. N., et al. 1979, ApJS, 39, 573

Markwardt, C. 2006, in The Transient Milky Way: a perspective for MIRAX, ed. F. D'Amico, J. Braga, \& R. Rothschild, AIP Conf. Proc., 840, 45

Markwardt, C. B., \& Swank, J. H. 2003a, ATel \#133

Markwardt, C. B., \& Swank, J. H. 2003b, ATel \#136

Markwardt, C. B., \& Swank, J. H. 2005, ATel \#414

Markwardt, C. B., Marshall, F. E., Swank, J. H., \& Cui, W. 1999, IAUC 7300

Markwardt, C. B., Swank, J. H., \& Marshall, F. E. 1999, IAUC 7120

Mas-Hesse, J. M., Giménez, A., Culhane, J. L., et al. 2003, A\&A, 411, L261

Masetti, N. 2002, A\&A, 381, L45

McClintock, J. E., \& Remillard, R. A. 2006, in Compact Stellar X-ray Sources, ed. W. H. G. Lewin, \& M. van der Klis (Cambridge University Press), 157

Molkov, S., Revnivtsev, M., Lutovinov, A., \& Sunyaev, R. 2005a, A\&A, 434, 1069

Molkov, S. V., Grebenev, S. A., \& Sunyaev, R. A. 2005b, ATel \#462

Mowlavi, N., Kuulkers, E., Rodriguez, J., et al. 2005, ATel \#453

Muno, M. P., Baganoff, F. K., Bautz, M. W., et al. 2003a, ApJ, 589, 225

Muno, M. P., Baganoff, F. K., \& Arabadjis, J. S. 2003b, ApJ, 598, 474

Muno, M. P., Pfahl, E., Baganoff, F. K., et al. 2005a, apJ, 622, L113

Muno, M. P., Lu, J. R., \& Baganoff, F. K. 2005b, ApJ, 633, 228

Natalucci, L., Bazzano, A., Cocchi, M., et al. 2004, A\&A, 416, 699

Orr, A., Falanga, M. Cocchi, M., Laurent, P., \& Goldwurm, A. 2004, in Proc. 5th INTEGRAL Science Workshop, The INTEGRAL Universe, ed. V. Schönfelder, G. Lichti, \& C. Winkler, ESA SP-552, 357

Paizis, A., Ebisawa, K., Tikkanen, T., et al. 2005, A\&A, 443, 599

Paizis, A., Farinelli, R., Titarchuk, L., et al. 2006, A\&A, 459, 187

Parmar, A. N., Kuulkers, E., Oosterbroek, T., et al. 2003, A\&A, 411, L421

Pellizza, L. J., Chaty, S., \& Negueruela, I. 2006, A\&A, 455, 653

Pereira, M. G., Braga, J., \& Jablonski, F. 1999, ApJ, 526, L105

Pietsch, W., Voges, W., Reppin, C., et al. 1980, ApJ, 237, 964

Piraino, S., Santangelo, A., Ferrigno, C., et al. 2004, in The X-ray Universe, Proc. 5th INTEGRAL workshop, ESA SP-552, 369

Pottschmidt, K., Chernyakova, M., Zdziarski, A. A., et al. 2006, in The X-ray Universe 2005, ESA SP-604, 283

Reynolds, A. P., Parmar, A. N., Hakala, P. J., et al. 1999, A\&AS, 134, 287

Remillard, R. A., Lin, D., Cooper, R. L., \& Narayan, R. 2006a, ApJ, 646, 407

Remillard, R., Levine, A. M., Morgan, E. H., Markwardt, C. B., \& Swank, J. H. 2006b, ATel \#714

Revnivtsev, M., Chernyakova, M., Capitanio, F., et al. 2003, ATel \#132

Revnivtsev, M. G., Sunyaev, R. A., Varshalovich, D. A., et al. 2004a, AstL, 30, 382

Revnivtsev, M. G., Sunyaev, R. A., Gilfanov, M. R., et al. 2004b, AstL, 30, 527

Rodriguez, J. 2003, ATel \#194

Rubin, B. C., Finger, M. H., Harmon, B. A., et al. 1996, ApJ, 459, 259

Rupen, M. P., Mioduszewski, A. J., \& Dhawan, V. 2005, ATel \#441

Sakano, M., Koyama, K., Murakami, H., Maeda, Y., \& Yamauchi, S. 2002, ApJS, 138,19

Shaposhnikov, N., Swank, J., Shrader, C. R., et al. 2006, ApJ, in press [arXiv: astro-ph/0609757]

Shaw, S. E., Kuulkers, E., Turler, M., et al. 2005a, ATel \#583

Shaw, S. E., Kuulkers, E., Oosterbroek, T., et al. 2005b, ATel \#615

Shaw, S., Kuulkers, E., Paizis, A., et al. 2005c, ATel \#442

Shaw, S. E., Zurita, J., Kuulkers, E., et al. 2006, ATel \#731

Skinner, G. K., Foster, A. J., Willmore, A. P., \& Eyles, C. J. 1990, MNRAS, 243, 72

Skinner, G. K., Willmore, A. P., Eyles, C. J., et al. 1987, Nature, 330, 544

Smith, D. M., Heindl, W. A., Markwardt, C. B., \& Swank, J. H. 2001, ATel \#66

Smith, D. M., Heindl, W. A., \& Swank, J. H. 2002, ApJ, 578, 129

Strohmayer, T., \& Bildsten, L. 2006, in Compact Stellar X-ray Sources, ed. W. H. G. Lewin, \& M. van der Klis, Cambridge University Press, 113

Sunyaev, R., Molkov, S., \& Lutovinov, A. 2003a, ATel \#187

Sunyaev, R. A., Grebenev, S. A., Lutovinov, A. A., et al. 2003b, ATel \#190

Swank, J. 2004, ATel \#301

Swank, J., \& Markwardt, C. 2001, in ASP Conf. Proc. 251, ed. H. Inoue, \& H. Kunieda, ASP, San Francisco, 94

Swank, J. H., Remillard, R., \& Markwardt, C. B. 2005, ATel \#576

Tarana, A., Bazzano, A., Ubertini, P., \& Zdziarski, A. A. 2006a, ApJ, in press [arXiv:astro-ph/0608599]

Tarana, A., Bazzano, A., Ubertini, P., \& Federici, M. 2006b, in proceedings of the 6th INTEGRAL Workshop The Obscured Universe, ed. R. Sunyaev, S. Grebenev, \& C. Winkler, ESA-SP 622, in press [arXiv:astro-ph/0610325] 
Tavani, M., \& Barret, D. 1997, in Proceedings of the Fourth Compton Symposium, ed. C. D. Dermer, M. S. Strickman, \& J. D. Kurfess, AIP Conf. Proc., 410, 75

Thompson, T. W. J., Tomsick, J. A., in 't Zand, J. J. M., Rothschild, R. E., \& Walter, R. 2006, ApJ, submitted [arXiv: astro-ph/0609424]

Turler, M., Shaw, S. E., Kuulkers, E., et al. 2006, ATel \#790

Ubertini, P., Lebrun, F., Di Cocco, G., et al. 2003, A\&A, 411, L131

van der Klis, M., Kitamoto, S., Tsunemi, H., \& Miyamoto, S. 1991, MNRAS, 248,751

Vedrenne, G., Roques, J.-P., Schönfelder, V., et al. 2003, A\&A, 411, L63

Walter, R., Bodaghee, A., Barlow, E. J., et al. 2004, ATel \#229
Walter, R., Zurita Heras, J., Bassani, L., et al. 2006, A\&A, 453, 133

Werner, N., in 't Zand, J. J. M., Natalucci, L., et al. 2004, A\&A, 416, 311

White, N. E., Becker, R. H., Boldt, E. A., et al. 1981, ApJ, 247, 994

Williams, O. R., Oosterbroek, T., Parmar, A. N., \& Winkler, C. 2004, in Proc. 5th INTEGRAL Science Workshop, The INTEGRAL Universe, ed. V. Schönfelder, G. Lichti, \& C. Winkler, ESA SP-552, 423

Wijnands, R., Miller, J. M., \& Wang, Q. D. 2002, ApJ, 579, 422

Wijnands, R., in 't Zand, J. J. M., Rupen, et al. 2006, A\&A, 449, 1117

Winkler, C., Courvoisier, T. J.-L., Di Cocco, G., et al. 2003, A\&A, 411, L1

Zurita, J., Bazzano, A., Brandt, S., et al. 2004, ATel \#248

Zurita Heras, J. A., De Cesare, G., Walter, R., et al. 2006, A\&A, 448, 26 
E. Kuulkers et al.: INTEGRAL Galactic bulge monitoring program, Online Material $p 1$

\section{Online Material}




\section{Appendix A: The Crab as the reference source}

In order to derive the average Crab rates we used the Crab calibration observations, performed during revolution 300 (March 2005; MJD 53457-53459), 365 (October 2005; MJD 53654) and 422 (March 2006; MJD 53822-53824). They were performed during the same seasons as our monitoring observations. We only used those observations with similar instrument settings as our monitoring observations; they were analysed in the same way as described for our program (Sect. 3). The JEM-X and IBIS/ISGRI light curves are shown in Fig. A.1. The IBIS/ISGRI $20-60 \mathrm{keV}$ and $60-150 \mathrm{keV}$ count rates are largely constant (i.e., within about $2 \%$ ) across the central part of the field of view (see Fig. A.2). The JEM-X count rates are constant within about $5 \%(3-10 \mathrm{keV})$ and $(10-25 \mathrm{keV})$. The average count rates in the JEM-X $3-10 \mathrm{keV}$ and $10-25 \mathrm{keV}$, and IBIS/ISGRI 20-60 keV and 60-150 keV, are $109.8 \pm 0.2 \mathrm{cts} \mathrm{s}^{-1}$, $46.0 \pm 0.1 \mathrm{cts} \mathrm{s}^{-1}, 164.13 \pm 0.05 \mathrm{cts} \mathrm{s}^{-1}$ and $43.11 \pm 0.04 \mathrm{cts} \mathrm{s}^{-1}$, respectively.

\section{Appendix B: IBIS/ISGRI 20-60 keV sensitivity}

The $5 \sigma$ sensitivity of our Galactic bulge monitoring observations for the IBIS/ISGRI instrument in the $20-60 \mathrm{keV}$ band was determined using Crab observations and as well as the monitoring observations themselves.

The Crab calibration observations, with exposure lengths in the range $1700-1800$ s (i.e., similar to our monitoring observations), were selected from revolutions 300, 365 and 422 (see also Appendix A). For each Crab detection, the value of the $1 \sigma$ uncertainty on the Crab flux detection was multiplied by 5 and scaled by the average Crab flux to give an estimate of the $5 \sigma$ sensitivity of the observations as a function of the off-axis angle (the angular distance of the Crab from the center of the IBIS/ISGRI field of view). These points are shown as the green triangles in Fig. B.1.

In order to increase the sampling of the sensitivity curve over a wider range of off-axis angles, the same exercise was performed by taking the $1 \sigma$ uncertainty on every detection made in the Galactic bulge observations, with similar exposure times of $1800 \mathrm{~s}$. These are shown as red dots in Fig. B.1. It can be clearly seen that estimating the sensitivity from all detections agrees well with the data from the Crab, although there is some evidence that the sensitivity for the Crab observations could be systematically lower (i.e., the limiting fluxes are higher) than the Galactic bulge observations by a small amount. This may be because the brightness of the Crab leads to an increase in the background of the IBIS/ISGRI images.

The top dashed line in Fig. B. 1 is a fit of an exponential curve to the data $\left(y=0.329 \mathrm{e}^{0.362 x}+21.2\right.$, where $y$ is the $5 \sigma$ sensitivity in mCrab and $x$ the off-axis angle in degrees). Below that the lower envelope of the points has been estimated to provide a lower limit to the sensitivity for a single 1800 s pointing; again an exponential has been used $\left(y=0.7 \mathrm{e}^{0.29 x}+18\right)$ and is shown by the continuous line.

An estimate of the sensitivity for a full hexagonal dither observation (i.e., $7 \times 1800 \mathrm{~s}$ observations) has been performed by scaling the latter curve by $\sqrt{ } 7$ (the signal-to-noise ratio scales with the square-root of the exposure time; see, e.g., Goldwurm et al. 2003); this is shown as a dotted line in Fig. B.1. Note that this should only be used as a guide to the observation sensitivity, since the hexagonal dither pattern means that each pointing within a pattern will have off-axis angles varying by $\pm 2^{\circ}$. This indicates a $5 \sigma$ sensitivity, in the $20-60 \mathrm{keV}$ band of about $7 \mathrm{mCrab}$ for on-axis observations, rising to $8 \mathrm{mCrab}$ at $5^{\circ}, 12 \mathrm{mCrab}$ at $10^{\circ}$ and above $27 \mathrm{mCrab}$ outside of $15^{\circ}$. We note that these sensitivity fluxes are consistent with the observed fluxes for sources detected around $5 \sigma$ in the mosaics per revolution. For more information on the IBIS/ISGRI sensitivities we refer to, e.g., Lebrun et al. (2003) and Bird et al. (2006).

\section{Appendix C: Mean fluxes from individual pointings versus average fluxes from overall mosaic image}

As noted in Sect. 4.2 the mean fluxes computed by averaging the flux values (weighted by their errors) from all the single pointings are different with respect to flux values obtained from the mosaic image of all pointings together. There are mainly two reasons for this. While the mosaic image merges all the single pointings of a source (even those pointings where the source is too weak to be detected and contributes with a negative fluctuation), the single-pointing averaged fluxes are obtained by averaging only those single pointings where the source had a non-negative significance (and hence flux) value. This results in an overestimate of the mean flux, especially for weak sources or (bright) transient sources which are off for some time. Additionally, in the mosaic images the counts associated to a source are spread around a single central peak, resulting in a better source location. However, the source flux is then also somewhat reduced (by $\simeq 10 \%$; see, e.g., Chernyakova 2005). In Fig. C.1 we show these effects by plotting the mosaic flux (from Table 3) versus the mean flux (from Table 6) values for those source where the significance value of detection is higher than 7. The continuous line represents where the mean flux equals the mosaic flux, whereas the dotted line represents where the mosaic flux is $10 \%$ lower than the mean flux. Permanently bright (typically $\gtrsim 20 \mathrm{mCrab}$ ) sources indeed show a mosaic flux which is about $10 \%$ lower than the mean flux. The weaker sources and bright transient sources (notably GRO J1655-40 and 1E 1740.7-2942) clearly show the additional offset, due to our method of averaging, in the mosaic flux with respect to the mean flux. 
E. Kuulkers et al.: INTEGRAL Galactic bulge monitoring program, Online Material $p 3$
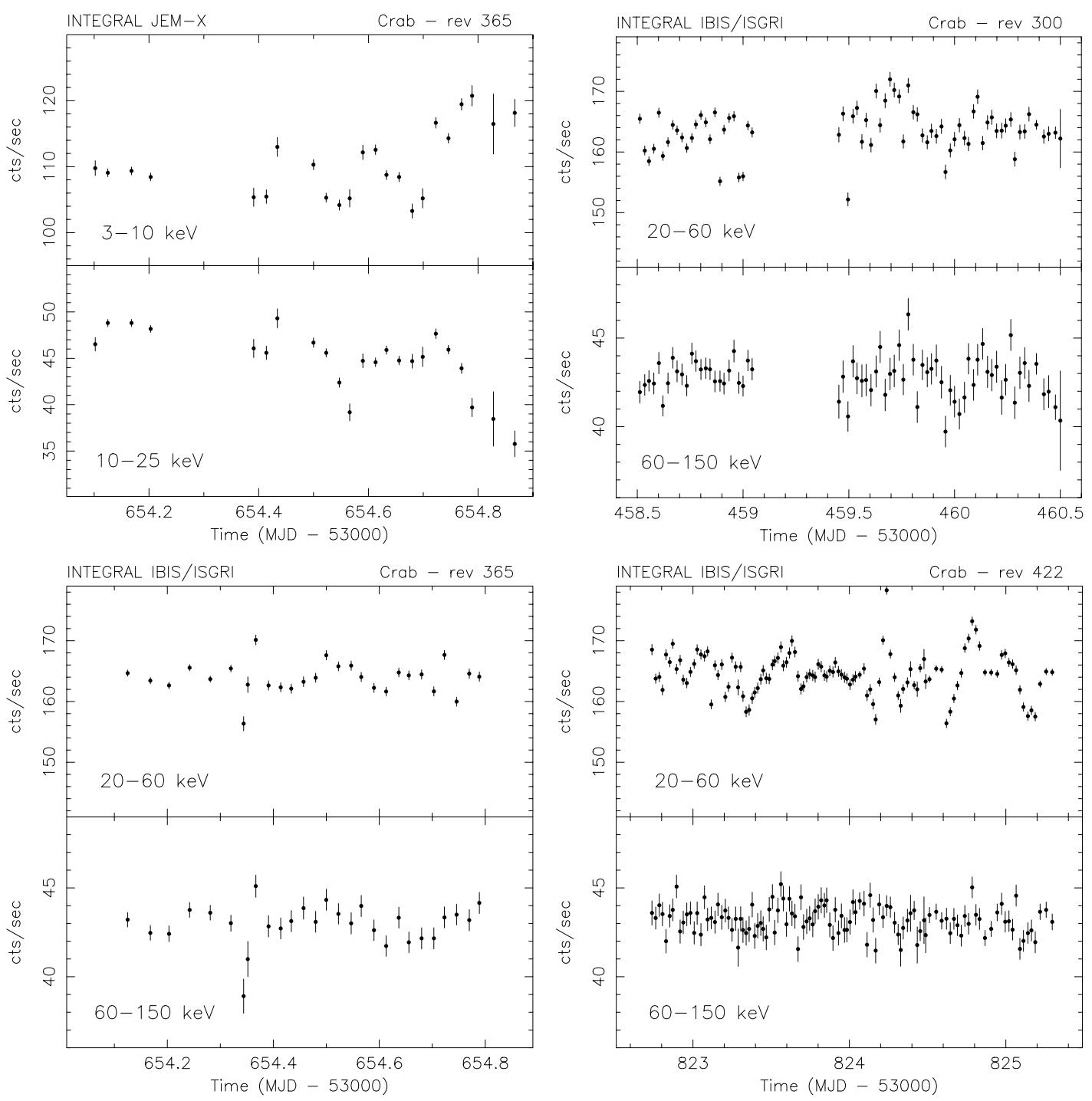

Fig. A.1. INTEGRAL JEM-X light curves of the Crab during the calibration in revolution 365 (top left) and IBIS/ISGRI light curves of the Crab during the calibration in revolutions 300 (top right), 365 (bottom left) and 422 (bottom right). Plotted per panel are the two energy bands used: JEM-X 3-10 keV (panel top), 10-25 keV (panel bottom), and IBIS/ISGRI 20-60 keV (panel top), 60-150 keV (panel bottom). 
E. Kuulkers et al.: INTEGRAL Galactic bulge monitoring program, Online Material $p 4$

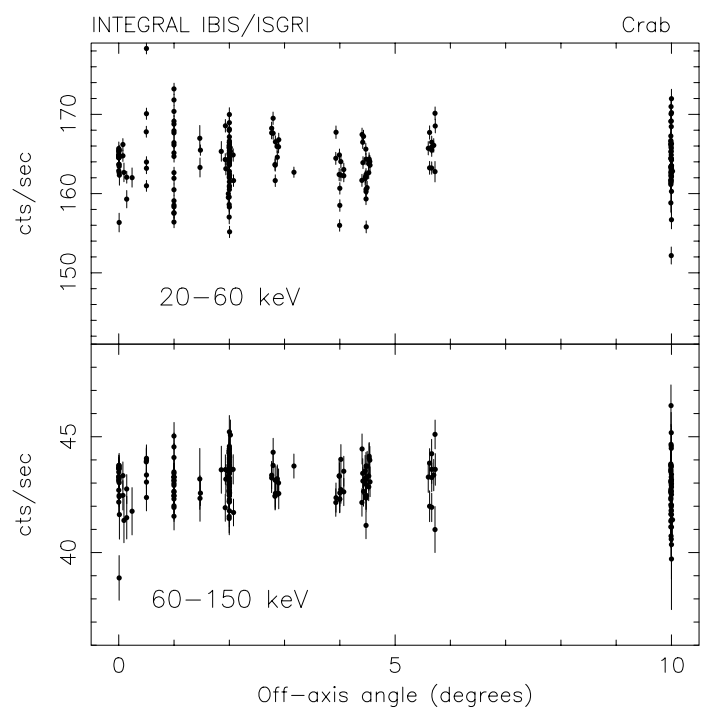

Fig. A.2. IBIS/ISGRI 20-60 keV (top) and 60-150 keV (bottom) Crab count rates as a function of the off-axis angle.

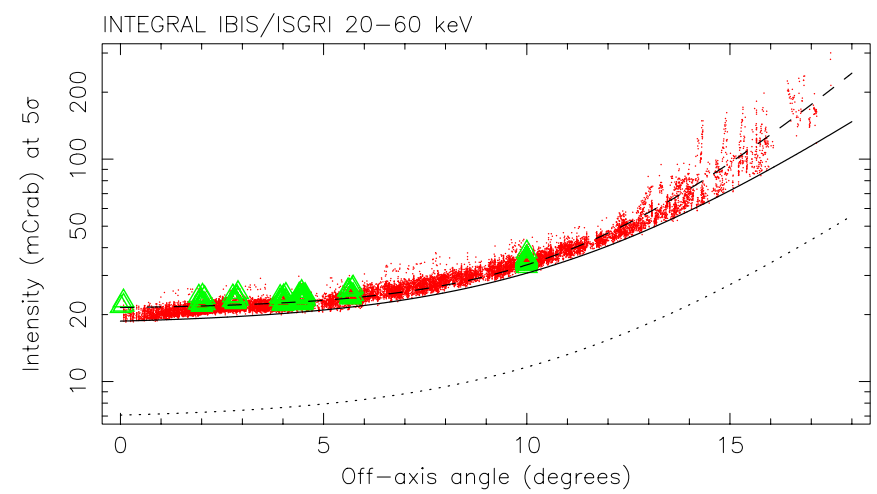

Fig. B.1. IBIS/ISGRI $20-60 \mathrm{keV} 5 \sigma$ sensitivity information. We refer to the text (Sect. B) for a detailed explanation of the various data points and curves.

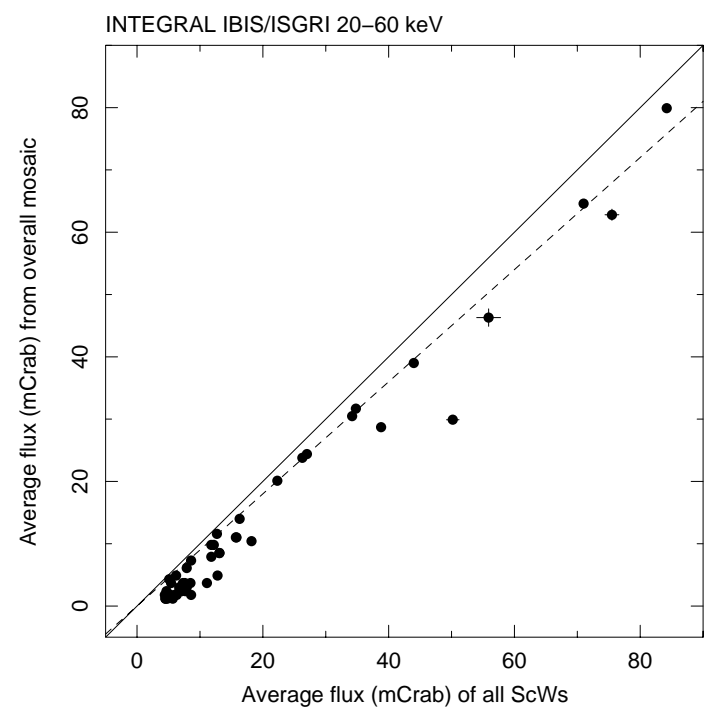

Fig. C.1. Average flux from the mosaic image of all pointings (ScWs) versus the mean flux over all single pointings $(20-60 \mathrm{keV})$. Data are taken from Tables 3 and 6; plotted are those sources which reached a significance higher than 7 . The values for which the mosaic average flux equals the mean flux are indicated by a continuous line; the dashed line indicates where the mosaic flux equals $90 \%$ of the mean flux. 